Prepared in cooperation with the National Climate Change and Wildlife Science Center

\title{
Stream Classification of the Apalachicola-Chattahoochee- Flint River System to Support Modeling of Aquatic Habitat Response to Climate Change
}

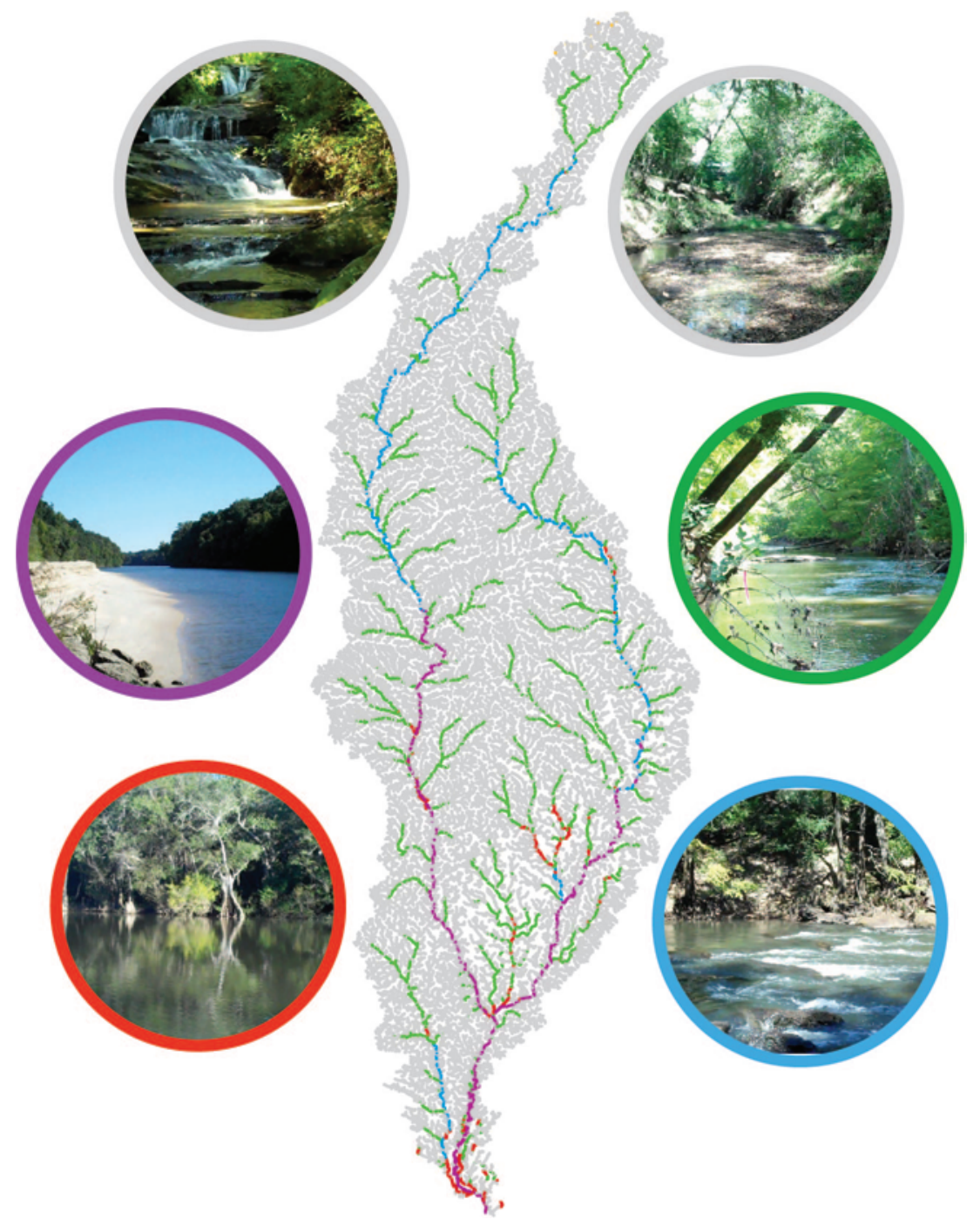

Scientific Investigations Report 2014-5080 
Cover. Photographs clockwise from top left: Horton Creek, Georgia; Little Bear Creek, Georgia; Big Creek, Georgia; Ichawaynochaway Creek, Georgia; Chattahoochee River, Georgia; and Chipola River, Florida.

All photographs by Roy Thorsby, formerly of U.S. Geological Survey. 


\section{Stream Classification of the Apalachicola- Chattahoochee-Flint River System to Support Modeling of Aquatic Habitat Response to Climate Change}

By Caroline M. Elliott, Robert B. Jacobson, and Mary C. Freeman

Prepared in cooperation with the National Climate Change and Wildlife Science Center

Scientific Investigations Report 2014-5080 


\title{
U.S. Department of the Interior SALLY JEWELL, Secretary
}

\section{U.S. Geological Survey Suzette M. Kimball, Acting Director}

\author{
U.S. Geological Survey, Reston, Virginia: 2014
}

For more information on the USGS - the Federal source for science about the Earth, its natural and living resources, natural hazards, and the environment, visit http://www.usgs.gov or call 1-888-ASK-USGS.

For an overview of USGS information products, including maps, imagery, and publications, visit http://www.usgs.gov/pubprod

To order this and other USGS information products, visit http://store.usgs.gov

Any use of trade, firm, or product names is for descriptive purposes only and does not imply endorsement by the U.S. Government.

Although this information product, for the most part, is in the public domain, it also may contain copyrighted materials as noted in the text. Permission to reproduce copyrighted items must be secured from the copyright owner.

Suggested citation:

Elliott, C.M., Jacobson, R.B., and Freeman, M.C., 2014, Stream classification of the Apalachicola-ChattahoocheeFlint River System to support modeling of aquatic habitat response to climate change: U.S. Geological Survey Scientific Investigations Report 2014-5080, 79 p., http://dx.doi.org/10.3133/sir20145080.

ISSN 2328-0328 (online) 


\section{Contents}

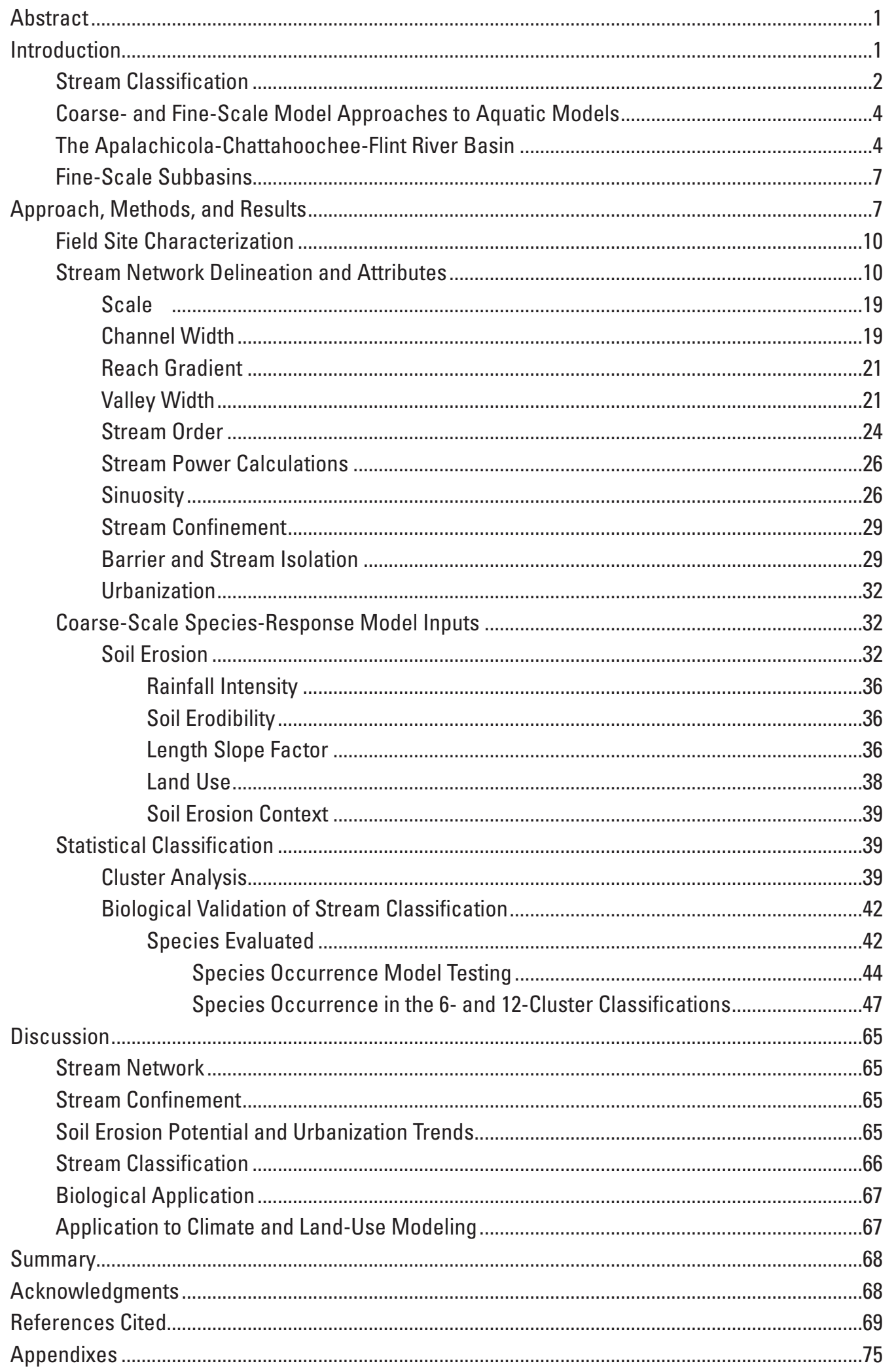




\section{Figures}

1. Maps showing physiographic provinces, major cities, reservoirs, major river basins, and principal streams of the Apalachicola-Chattahoochee-Flint River Basin in Georgia, Alabama, and Florida, and the Upper Roanoke River Basin in Virginia

2. Maps showing coarse- and fine-scale stream networks for the basin, segment, and reach scales for hydrologic and aquatic biota models in the Apalachicola-Chattahoochee-Flint River Basin and the Upper Roanoke River Basin .....5

3. Maps showing regional geology of the Apalachicola-Chattahoochee-Flint River Basin in Georgia, Alabama, and Florida, and the Upper Roanoke River Basin in Virginia.

4. Maps showing the digital elevation model derived stream network for the Upper Chattahoochee River, Chestatee River, Potato Creek, and Upper Roanoke River Basins.

5. Maps showing the digital elevation model derived stream network for the Spring Creek, Ichawaynochaway Creek, and Chipola River Basins.

6. Maps showing the digital elevation model stream network and the high- and medium-resolution National Hydrography Datasets for the

Apalachicola-Chattahoochee-Flint River Basin and the Potato Creek Basin....

7. Maps showing drainage basin area, in square kilometers, associated with fine-scale digital elevation model derived drainage network reaches for the Apalachicola-Chattahoochee-Flint River Basin stream network with details shown for the Potato Creek Basin .

8. Maps and graphs showing cross-section geometry derived from the light detection and ranging digital elevation model dataset for the Potato Creek Basin.....

9. Graph showing modeled bankfull channel width comparison from field estimates, U.S. Geological Survey streamflow gaging station data analysis for the Apalachicola-Chattahoochee-Flint River Basin, and light detection and ranging digital elevation model measurements for the Potato Creek Basin.

10. Maps showing modeled channel width, in meters, for the Apalachicola-Chattahoochee-Flint River Basin stream network with details shown for the Potato Creek Basin .

11. Maps showing mean reach gradient for the Apalachicola-Chattahoochee-Flint River Basin stream network with details shown for the Potato Creek Basin..

12. Graph showing modeled valley width comparison with field measurements throughout the Apalachicola-Chattahoochee-Flint River Basin, digital-elevation model, and light detection and ranging digital elevation model measurements in the Potato Creek Basin

13. Maps showing modeled valley width, in meters, for the Apalachicola-Chattahoochee-Flint River Basin stream network with details shown for the Potato Creek Basin .

14. Maps showing Strahler stream order for the Apalachicola-Chattahoochee-Flint River Basin stream network with details shown for the Potato Creek Basin..

15. Maps showing unit stream power for the Apalachicola-Chattahoochee-Flint River Basin stream network with details shown for the Potato Creek Basin.

16. Maps showing sinuosity for the Apalachicola-Chattahoochee-Flint River Basin stream network with details shown for the Potato Creek Basin

17. Maps showing stream confinement for the Apalachicola-Chattahoochee-Flint River Basin stream network with details shown for the Potato Creek Basin. 
18. Maps showing ratio of valley width to channel width for the

Apalachicola-Chattahoochee-Flint River Basin stream network with details shown

for the Potato Creek Basin

19. Maps showing stream-segment barriers and isolation for the Upper

Chattahoochee River, Chestatee River, Potato Creek, and Upper Roanoke River

Basins

20. Maps showing stream-segment barriers and isolation for the Spring Creek, Ichawaynochaway Creek, and Chipola River Basins.

21. Graph showing comparisons in the percent of stream segments that contain barriers or are isolated for the seven fine-scale model basins

22. Maps showing the Apalachicola-Chattahoochee-Flint River Basin with urban land classified with a probability of greater than 50 percent in 2010 and 2100

23. Graph showing trends in percent of land classified as urban for the seven fine-scale model basins from 2010 to 2100 .

24. Maps showing revised Universal Soil Loss Equation soil-erosion geographic information system model developed for the Apalachicola-Chattahoochee-Flint River Basin

25. Maps showing revised Universal Soil Loss Equation estimates of potential soil loss summarized by hydrologic response unit in the Apalachicola-Chattahoochee-Flint River Basin

26. Diagram showing principal component analysis for seven geomorphic characteristics of a randomly selected subset of stream reaches in the Apalachicola-Chattahoochee-Flint River Basin .

27. Scatterplots and histograms of seven geomorphic characteristics for a randomly selected subset of stream reaches in the Apalachicola-Chattahoochee-Flint River Basin .....

28. Scatterplots and histograms for channel width, valley width, and gradient measured at a randomly selected subset of stream reaches in the Apalachicola-Chattahoochee-Flint River Basin

29. Graph showing changes in the sum-of-squares differences within and between clusters as number of clusters changes

30. Graphs showing clustering results from the K-means procedure at 6 and 12 clusters

31. Diagram showing canonical scores for the 6-cluster classification of stream reaches within the Apalachicola-Chattahoochee-Flint River Basin.

32. Diagram showing canonical scores for the 12-cluster classification of stream reaches within the Apalachicola-Chattahoochee-Flint River Basin.

33. Maps showing spatial distribution of stream reaches within the Apalachicola-Chattahoochee-Flint River Basin for the 6-cluster classification

34. Maps showing spatial distribution of stream reaches within the Apalachicola-Chattahoochee-Flint River Basin for the 12-cluster classification

35. Graphs showing distributions by cluster of six geomorphic characteristics of stream reaches within the Apalachicola-Chattahoochee-Flint River Basin for the 6-cluster classific ation .

36. Graphs showing distributions by cluster of six geomorphic characteristics of stream reaches within the Apalachicola-Chattahoochee-Flint River Basin for the 12-cluster classification

37. Map showing field-site locations within the Apalachicola-Chattahoochee-Flint River Basin, example photographs, and the 6-cluster classification 
38. Map showing field-site locations within the Apalachicola-Chattahoochee-Flint River Basin, example photographs, and the 12-cluster classification

39. Maps showing species distributions of the Halloween darter (Percina crypta) and blackbanded darter (Percina nigrofaciata) and the 6-cluster classification of stream reaches in the Apalachicola-Chattahoochee-Flint River Basin with details shown in three regions

40. Maps showing species distributions of Halloween darter (Percina crypta) and blackbanded darter (Percina nigrofaciata) and the 12-cluster classification of stream reaches in the Apalachicola-Chattahoochee-Flint River Basin with details shown in three regions

41. Maps showing species distributions of bluestripe shiner (Cyprinella callitaenia) and blacktail shiner (Cyprinella venusta) and the 6-cluster classification of stream reaches in the Apalachicola-Chattahoochee-Flint River Basin with details shown in three regions

42. Maps showing species distributions of bluestripe shiner (Cyprinella callitaenia) and blacktail shiner (Cyprinella venusta) and the 12-cluster classification of stream reaches in the Apalachicola-Chattahoochee-Flint River Basin with details shown in three regions 


\section{Tables}

1. Geomorphic variables and basin summary data for the

Apalachicola-Chattahoochee-Flint River Basin and six fine-scale model basins nested within the Apalachicola-Chattahoochee-Flint River Basin as well as the Upper Roanoke River Basin in Virginia

2. Field measurements of selected geomorphic and habitat variables for 59 streams in the Apalachicola-Chattahoochee-Flint River Basin .

3. Geographic information system derived estimates of selected geomorphic variables for 59 streams in the Apalachicola-Chattahoochee-Flint River Basin, based on 30-meter digital elevation model data, and channel classification assignments based on 6 - and 12-cluster classification models

4. Measured erosion rates and ranges for native vegetation, geologic regions, and agricultural practices.

5. Jackknife validation for the 6 - and 12-cluster classifications of streams in the Apalachicola-Chattahoochee-Flint River Basin...

6. Statistical summary by cluster for selected geomorphic variables for streams in the Apalachicola-Chattahoochee-Flint River Basin for the 6- and 12-cluster classifications

7. Descriptive summaries for the 6-and 12-cluster classifications of streams in the Apalachicola-Chattahoochee-Flint River Basin..

8. Comparative support for logistic regression models for the Halloween darter and bluestripe shiner occurrence based on geomorphology variables and classes from a 6 -cluster classification

9. Regression coefficient estimates for best-supported logistic regression models of the occurrence of the Halloween darter and bluestripe shiner based on geomorphology variables and on a 6-cluster classification for the Apalachicola-Chattahoochee-Flint River Basin .

10. Prediction error rates for best-supported models of occurrence of Halloween darter and bluestripe shiner, based on either individual geomorphic variables or stream classification.

11. Total number of segments in each cluster of the 6-cluster classification scheme, number of segments with known Halloween darter and Bluestripe shiner occurrence, and mean predicted probability of occurrence based on the best-supported geomorphic and classification models using all observations.....

12. Species distributions from collection records by cluster for the 6- and 12-cluster classifications of streams within the Apalachicola-Chattahoochee-Flint River Basin for two darters and two minnows..............................................................................60

1-1. Reach-scale layer attributes ....................................................................................

2-1. Segment-scale layer attributes ......................................................................................77

3-1. Land-use C-factor assignments used in Revised Universal Soil Loss

Equation model. 


\section{Conversion Factors and Datums}

Inch/Pound to SI

\begin{tabular}{|c|c|c|}
\hline Multiply & By & To obtain \\
\hline \multicolumn{3}{|c|}{ Length } \\
\hline foot $(\mathrm{ft})$ & 0.3048 & meter $(\mathrm{m})$ \\
\hline mile (mi) & 1.609 & kilometer (km) \\
\hline yard (yd) & 0.9144 & meter $(\mathrm{m})$ \\
\hline \multicolumn{3}{|c|}{ Area } \\
\hline acre & 4,047 & square meter $\left(\mathrm{m}^{2}\right)$ \\
\hline acre & 0.004047 & square kilometer $\left(\mathrm{km}^{2}\right)$ \\
\hline square mile $\left(\mathrm{mi}^{2}\right)$ & 2.590 & square kilometer $\left(\mathrm{km}^{2}\right)$ \\
\hline \multicolumn{3}{|c|}{ Flow rate } \\
\hline acre-foot per day (acre-ft/d) & 0.01427 & cubic meter per second $\left(\mathrm{m}^{3} / \mathrm{s}\right)$ \\
\hline cubic foot per second $\left(\mathrm{ft}^{3} / \mathrm{s}\right)$ & 0.02832 & cubic meter per second $\left(\mathrm{m}^{3} / \mathrm{s}\right)$ \\
\hline
\end{tabular}

SI to Inch/Pound

\begin{tabular}{|c|c|c|}
\hline Multiply & By & To obtain \\
\hline \multicolumn{3}{|c|}{ Length } \\
\hline meter $(\mathrm{m})$ & 3.281 & foot $(\mathrm{ft})$ \\
\hline kilometer (km) & 0.6214 & mile (mi) \\
\hline kilometer (km) & 0.5400 & mile, nautical (nmi) \\
\hline meter $(\mathrm{m})$ & 1.094 & yard (yd) \\
\hline \multicolumn{3}{|c|}{ Area } \\
\hline square meter $\left(\mathrm{m}^{2}\right)$ & 0.0002471 & acre \\
\hline square kilometer $\left(\mathrm{km}^{2}\right)$ & 247.1 & acre \\
\hline square kilometer $\left(\mathrm{km}^{2}\right)$ & 0.3861 & square mile $\left(\mathrm{mi}^{2}\right)$ \\
\hline \multicolumn{3}{|c|}{ Flow rate } \\
\hline cubic meter per second $\left(\mathrm{m}^{3} / \mathrm{s}\right)$ & 70.07 & acre-foot per day (acre-ft/d) \\
\hline cubic meter per second $\left(\mathrm{m}^{3} / \mathrm{s}\right)$ & 35.31 & cubic foot per second $\left(\mathrm{ft}^{3} / \mathrm{s}\right)$ \\
\hline millimeter per year $(\mathrm{mm} / \mathrm{yr})$ & 0.03937 & inch per year (in/yr) \\
\hline \multicolumn{3}{|c|}{ Density } \\
\hline kilograms per cubic meter $\left(\mathrm{kg} / \mathrm{m}^{3}\right)$ & 0.0624 & pound per cubic foot $\left(\mathrm{lb} / \mathrm{ft}^{3}\right)$ \\
\hline
\end{tabular}

Temperature in degrees Celsius $\left({ }^{\circ} \mathrm{C}\right)$ may be converted to degrees Fahrenheit $\left({ }^{\circ} \mathrm{F}\right)$ as follows:

${ }^{\circ} \mathrm{F}=\left(1.8 x^{\circ} \mathrm{C}\right)+32$

Temperature in degrees Fahrenheit $\left({ }^{\circ} \mathrm{F}\right)$ may be converted to degrees Celsius $\left({ }^{\circ} \mathrm{C}\right)$ as follows:

${ }^{\circ} \mathrm{C}=\left({ }^{\circ} \mathrm{F}-32\right) / 1.8$ 


\section{Abbreviations}

\begin{tabular}{ll} 
AIC & Akaike Information Criterion \\
DEM & digital elevation model \\
d-link & downstream link magnitude \\
GIS & geographic information system \\
HRU & hydrologic response unit \\
LIDAR & light detection and ranging \\
NAIP & National Aerial Inventory Program \\
NED & National Elevation Dataset \\
NHD & National Hydrography Dataset \\
NID & National Inventory of Dams \\
NLCD & National Land Cover Dataset \\
NRCS & Natural Resource Conservation Service \\
NWI & National Wetlands Inventory \\
PCA & principal component analysis \\
PRMS & precipitation-runoff modeling system \\
RUSLE & revised universal soil loss equation \\
SERAP & Southeast Regional Assessment Project \\
USGS & U.S. Geological Survey \\
USLE & universal soil loss equation \\
& \\
\hline
\end{tabular}





\title{
Stream Classification of the Apalachicola-Chattahoochee- Flint River System to Support Modeling of Aquatic Habitat Response to Climate Change
}

\author{
By Caroline M. Elliott, Robert B. Jacobson, and Mary C. Freeman
}

\section{Abstract}

A stream classification and associated datasets were developed for the Apalachicola-Chattahoochee-Flint River Basin to support biological modeling of species response to climate change in the southeastern United States. The U.S. Geological Survey and the Department of the Interior's National Climate Change and Wildlife Science Center established the Southeast Regional Assessment Project (SERAP) which used downscaled general circulation models to develop landscape-scale assessments of climate change and subsequent effects on land cover, ecosystems, and priority species in the southeastern United States. The SERAP aquatic and hydrologic dynamics modeling efforts involve multiscale watershed hydrology, stream-temperature, and fish-occupancy models, which all are based on the same stream network. Models were developed for the Apalachicola-Chattahoochee-Flint River Basin and subbasins in Alabama, Florida, and Georgia, and for the Upper Roanoke River Basin in Virginia.

The stream network was used as the spatial scheme through which information was shared across the various models within SERAP. Because these models operate at different scales, coordinated pair versions of the network were delineated, characterized, and parameterized for coarse- and fine-scale hydrologic and biologic modeling.

The stream network used for the SERAP aquatic models was extracted from a 30-meter (m) scale digital elevation model (DEM) using standard topographic analysis of flow accumulation. At the finer scale, reaches were delineated to represent lengths of stream channel with fairly homogenous physical characteristics (mean reach length $=350 \mathrm{~m}$ ). Every reach in the network is designated with geomorphic attributes including upstream drainage basin area, channel gradient, channel width, valley width, Strahler and Shreve stream order, stream power, and measures of stream confinement. The reach network was aggregated from tributary junction to tributary junction to define segments for the benefit of hydrological, soil erosion, and coarser ecological modeling. Reach attributes are summarized for each segment. In six subbasins segments are assigned additional attributes about barriers (usually impoundments) to fish migration and stream isolation. Segments in the six sub-basins are also attributed with percent urban area for the watershed upstream from the stream segment for each decade from 2010-2100 from models of urban growth.

On a broader scale, for application in a coarse-scale species-response model, the stream-network information is aggregated and summarized by 256 drainage subbasins (Hydrologic Response Units) used for watershed hydrologic and stream-temperature models. A model of soil erodibility based on the Revised Universal Soil Loss Equation also was developed at this scale to parameterize a model to evaluate stream condition.

The reach-scale network was classified using multivariate clustering based on modeled channel width, valley width, and mean reach gradient as variables. The resulting classification consists of a 6-cluster and a 12-cluster classification for every reach in the Apalachicola-Chattahoochee-Flint Basin. We present an example of the utility of the classification that was tested using the occurrence of two species of darters and two species of minnows in the Apalachicola-Chattahoochee-Flint River Basin, the blackbanded darter and Halloween darter, and the bluestripe shiner and blacktail shiner.

\section{Introduction}

The Southeast Regional Assessment Project (SERAP) is a collaborative effort by the U.S. Geological Survey (USGS) and the Department of the Interior's National Climate Change and Wildlife Science Center involving USGS scientists and partners including the Fish and Wildlife Service's Landscape Conservation Cooperatives, to provide a regional assessment of the potential effects of climate and land-use change. SERAP used downscaled general circulation models to develop assessments of climate change and subsequent effects on land cover, ecosystems, and priority species in the southeastern United States (Dalton and Jones, 2010). The intention of SERAP was to begin to integrate regionally downscaled probabilistic climate change predictions, terrestrial and coastal assessments, 
and a multiresolution assessment of potential climate change effects on hydrologic and aquatic resource modeling using tools from traditionally distinct scientific disciplines with the goal of exploring optimal conservation strategies to cope with climate change (Dalton and Jones, 2010).

A stream classification can provide a framework to filter hydrologic data into characteristic hydraulic responses for different types of streams that may have varying responses to climate and landscape change. The stream classification developed for the Apalachicola-Chattahoochee-Flint River Basin is designed to incorporate the inherent spatial variability of stream systems in real landscapes to enable an understanding of ecological responses to climate change and land-use change. The utility of stream classification is based on two assumptions: (1) landform data (for example, stream slope, confinement, geology) can be used to predict variation in stream characteristics at the stream reach or segment level (Montgomery, 1999; Rabeni and Sowa, 2002), and (2) stream characteristics are predictive of how fish populations respond to changes in flow variability (Poff, 1997; Walters and others, 2003; Peterson and others, 2009; McCargo and Peterson, 2010; Freeman and others, 2012). The study area stream network was used as the spatial scheme through which information was shared across the various hydrologic and biologic models within SERAP. Because these models operate at different scales, a coordinated pair of versions of the network was parameterized for use in coarse- and fine-scale hydrologic and biologic model applications.

This report describes methods to delineate and classify the stream network, particularly physical stream-channel habitat components that were developed for modeling freshwater fish and mussel population responses to climate and landscape change across multiple scales (Peterson and others, 2010; Freeman and others, 2012). This report describes the physicalhabitat data developed for SERAP models for the Apalachicola-Chattahoochee-Flint River Basin in Alabama, Florida, and Georgia, and for the Upper Roanoke River Basin in Virginia (fig. 1). We describe the physical stream-channel habitat components used in the characterization of these networks and the assessment of stream-channel condition. We also document the methods and data used to develop a stream classification and present a test of its utility based on species distributions.

\section{Stream Classification}

Stream classifications are used for a variety of purposes, including the simplification of complex systems for communication and management, stratification for monitoring-network design, and prediction of stream or biotic response to changes in hydrology, climate, or land use. Stream classification schemes vary widely (Kondolf and others, 2003) and include descriptive (Leopold and Wolman, 1957; Nanson and Croke,
1992), conceptual (Frissell and others, 1986; Thorp and others, 2006), and statistical classifications (Schmitt and others, 2007; Snelder and others, 2007; McManamay and others, 2011). Stream classes or categories typically are based on combinations of geomorphic, geologic, temperature, chemical, hydrological, or biological characteristics, such as macroinvertebrate or fish communities.

Stream classification schemes at the large drainagebasin or regional scale are becoming increasingly common. Although most schemes focus on hydrologic conditions, based on the assumption that flow regime is the "master" variable in stream ecology (Poff and others, 1997), some classification schemes also recognize that other geomorphic or physiographic characteristics mediate hydrologic effects (Poff and others, 2009). Region-wide or landscape-scale classifications include the River Styles Framework in Australia (Brierley and Fryirs, 2005), the River Environment Classification in New Zealand (Snelder and others, 2005), the Northeast Aquatic Habitat Classification System (Olivero and Anderson, 2008), and the North American Fish Habitat Condition Assessment (Wang and others, 2011).

For the Apalachicola-Chattahoochee-Flint River Basin classification, the objective was to provide a framework to link modeled stream discharges to different physically based stream classes that would have characteristic hydraulic responses, aquatic habitat availability, and sensitivity to climate and landscape change. We chose to use physical characteristics available at the drainage-basin scale for the classification, similar to efforts in the Pacific Northwest, primarily for salmonid habitats (Benda and others, 2007). Geomorphic variables such as gradient, drainage basin area, and channel width were generated at the reach scale for the entire Apalachicola-Chattahoochee-Flint network to develop the classification. Reach-based stream-network units subsequently were aggregated up to the segment scale for use in the fine-scale metapopulation models for guilds of fish and mussels, and to the subbasin level for the coarse-scale species-response model.

The stream networks for the ApalachicolaChattahoochee-Flint and Upper Roanoke River Basins are intended to serve as templates upon which modeled hydrologic changes are filtered into physical-habitat responses, and subsequently, into biotic responses. In heterogeneous terrain like the Apalachicola-Chattahoochee-Flint River Basin, stream morphology is thought to be an important mediator of flow regime alteration and a factor that needs to be accounted for to understand effects of climate, land-use, and water-use changes on stream biota (Poff and others, 2009). The stream classification presented in this report is based on the assumption that landscape-scale characteristics can be usefully quantified and analyzed in a geographic information system (GIS) and that the landscape-scale analysis will be predictive of the spatial distribution of stream classes at the reach scale. 

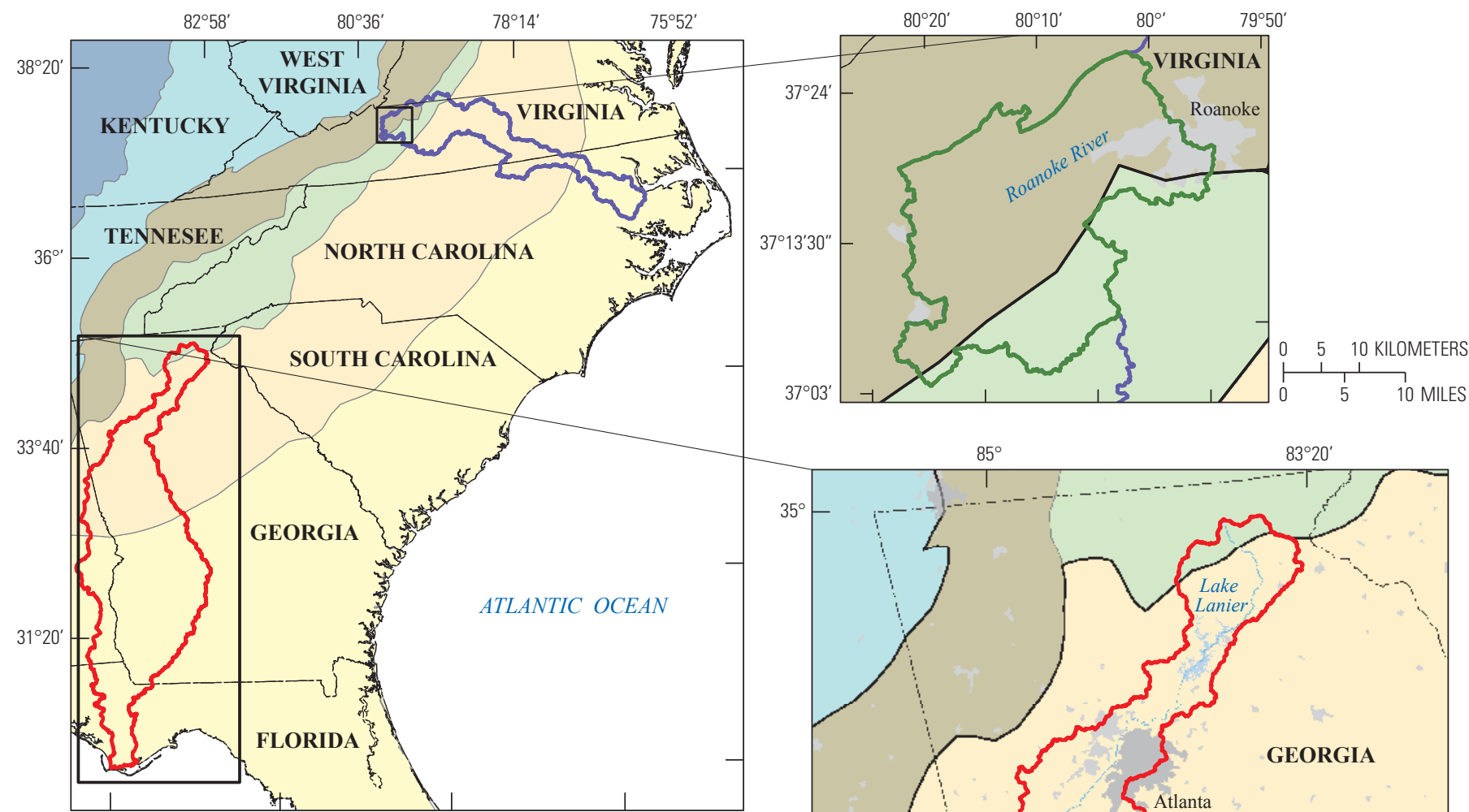

Base modified from U.S. Geological Survey digital data

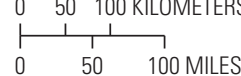

\section{EXPLANATION}

Physiographic province

(Fenneman, 1938)

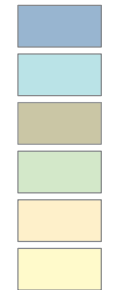

Interior Low Plateau

Appalachian Plateau

Valley and Ridge

Blue Ridge

Piedmont

Coastal Plain

Water body

Urban area

River basin boundary

A Albemarle-Pamlico

_ Apalachicola-Chattahoochee-Flint

- Upper Roanoke

Figure 1. Physiographic provinces, major cities, reservoirs, major river basins, and principal streams of the ApalachicolaChattahoochee-Flint River Basin in Georgia, Alabama, and Florida, and the Upper Roanoke River Basin in Virginia.
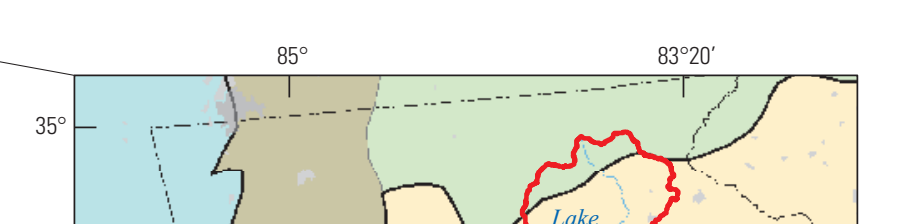

ins

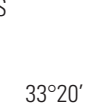


Stream Classification of the Apalachicola-Chattahoochee-Flint River System

\section{Coarse- and Fine-Scale Model Approaches to Aquatic Models}

The SERAP hydrologic and biologic models incorporate a multiple-scale approach consisting of a coarse-scale speciesresponse model for the entire Apalachicola-ChattahoocheeFlint River Basin and fine-scale metapopulation models for six smaller subbasins and the Upper Roanoke River Basin (fig. 2) (Peterson and others, 2010; LaFontaine and others, 2013). This two-tiered approach allows for characterization at the regional scale while providing information at the finer scale to help resolve variability in aquatic habitats and biotic responses.

All coarse- and fine-scale hydrologic models use the Precipitation-Runoff Modeling System (PRMS), a deterministic, distributed-parameter, physical process based modeling system well suited to evaluate streamflow responses to climate and land-use change scenarios (Leavesley, 1983). A hydrologic model previously was developed for the Flint River Basin (not on map) using PRMS to explore the hydrologic effects of urbanization and climate change (Viger and others, 2010; Viger and others, 2011).

The coarse-scale PRMS hydrologic model development and calibration is described in detail in LaFontaine and others (2013). The coarse-scale species-response models use PRMS and climate model output for baseline (defined as 1950-2000) and future (2050-2100) periods to explore effects of climate change on key drivers of stream and population dynamics (Peterson and others, 2010; Freeman and others, 2011; Peterson and others, 2011b). Habitat and biotic responses to changes in factors such as flow variability, flood duration, sediment supply, and drought frequency are designed to be estimated in a Bayesian Belief Network framework and parameterized using expert opinion (Marcot and others, 2001; Rieman and others, 2001). Applied at the subwatershed scale, the species-response models are intended to identify changes in occurrence probabilities for fish and mussel species having particular traits (Frimpong and Angermeier, 2009) throughout the Apalachicola-Chattahoochee-Flint River Basin for alternative climate futures.

The fine-scale model application consists of seven higher-resolution PRMS watershed and metapopulation dynamics models for fishes (Freeman and others, 2012). Six of the fine-scale model basins are within the ApalachicolaChattahoochee-Flint River Basin; one basin is located in the Albermarle-Pamlico Basin in Virginia (figs. 1-2). The finescale hydrologic models simulate streamflow dynamics under varying climate and land-use scenarios, and the occupancy models simulate the dynamics of colonization, reproduction, and persistence of individual species of fish and mussels within stream segments for yearly time-steps (Ruiz and Peterson, 2007; Peterson and others, 2010; Peterson and others, 2011a; Freeman and others, 2012). A prototype fine-scale model using PRMS and the hydrologic and aquatic speciesresponse modeling approach was previously developed for most of the Potato Creek Basin (Freeman and others, 2012).

\section{The Apalachicola-Chattahoochee-Flint River Basin}

The Apalachicola-Chattahoochee-Flint River Basin has a drainage area of more than 50,000 square kilometers $\left(\mathrm{km}^{2}\right)$; covers parts of Georgia, Alabama, and Florida; and has 1,300 meters $(\mathrm{m})$ of vertical relief from the Blue Ridge Mountain Province to the Gulf of Mexico (Couch and others, 1996) (fig. 1). The Apalachicola-Chattahoochee-Flint River Basin, along with other basins of the Eastern Gulf, is considered one of the most biologically diverse in North America, owing to its climate, geologic, and physical diversity (Lydeard and Mayden, 1995; Ward and others, 2005). The basin supports a variety of endemic (present only in the ApalachicolaChattahoochee-Flint River Basin) freshwater fish (Warren and others, 2000) and imperiled mussels (Brim Box and Williams, 2000).

Streams in the Apalachicola-Chattahoochee-Flint River Basin flow through three physiographic provinces, the Blue Ridge, Piedmont, and Coastal Plain (Fenneman, 1938). The Apalachicola-Chattahoochee-Flint River Basin has diverse geology ranging from metamorphic rocks in the Blue Ridge and Piedmont to sediments in the Coastal Plain (fig. 3). The Blue Ridge and Piedmont Provinces are underlain with generally resistant Precambrian Paleozoic igneous and metamorphic crystalline rocks, primarily granite, schist, gneiss, and quartzite (Garrity and Soller, 2009). The Blue Ridge Province has steep mountainous terrain and high-gradient bedrock streams with narrow valleys and boulder, gravel, and cobble substrates. The Piedmont begins in the foothills of the mountains and consists of mostly hilly to rolling terrain. The "fall line" forms the boundary between the Piedmont and Coastal Plain, and represents the Cretaceous-age shoreline where stream gradients are steep (Ward and others, 2005). The Coastal Plain generally consists of a dissected hilly region downstream from the fall line, a karst region called the Dougherty Plain, and a low-lying coastal region (fig. 3) (Couch and others, 1996). Streams in the Coastal Plain generally are low gradient and flow through Cenozoic sediments. There are extensive wetlands in flood plains of streams and rivers in the Coastal Plain in the Flint and Apalachicola River Basins.

The principal rivers of the Apalachicola-ChattahoocheeFlint River Basin are fragmented and altered by human activity to varying degrees. The headwaters of the upper Chattahoochee River are in the steep terrain of the Blue Ridge, and the Chattahoochee River is largely free flowing upstream of Lake Lanier, a large reservoir upstream of Atlanta, Georgia. Downstream, the Chattahoochee River is fragmented by 13 large dams and reservoirs in the Piedmont and Coastal Plain (Couch and others, 1996). The headwaters of the Flint River are in the Piedmont, just south of Atlanta, Georgia, and have a steeper gradient through the fall line with frequent rapids and shoals, and reaches with bedrock-constricted valleys and bedrock and cobble substrates. Downstream from the fall line, the middle Flint River has wide flood plains and sandy banks (Ward and others, 2005). The lower Flint River 
Apalachicola-Chattahoochee-Flint River Basin coarse- and fine-scale networks

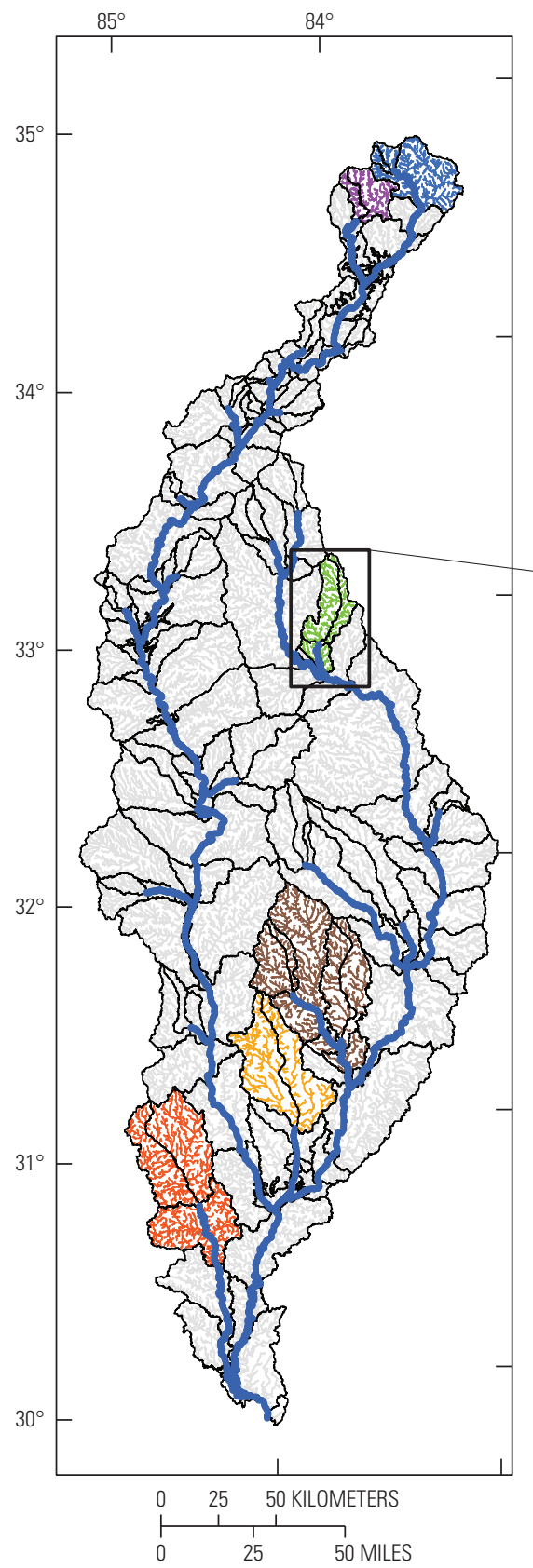

Upper Roanoke River Basin

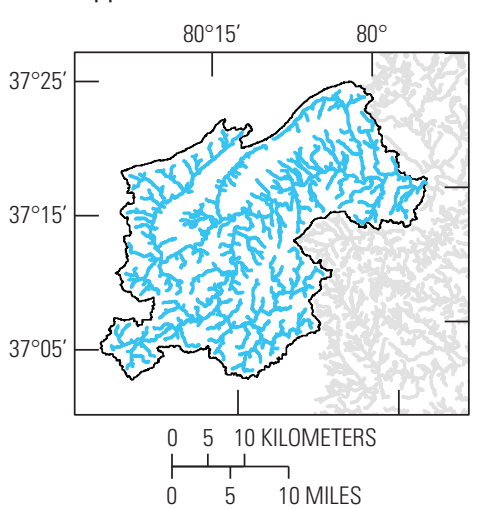

Potato Creek fine-scale model basin
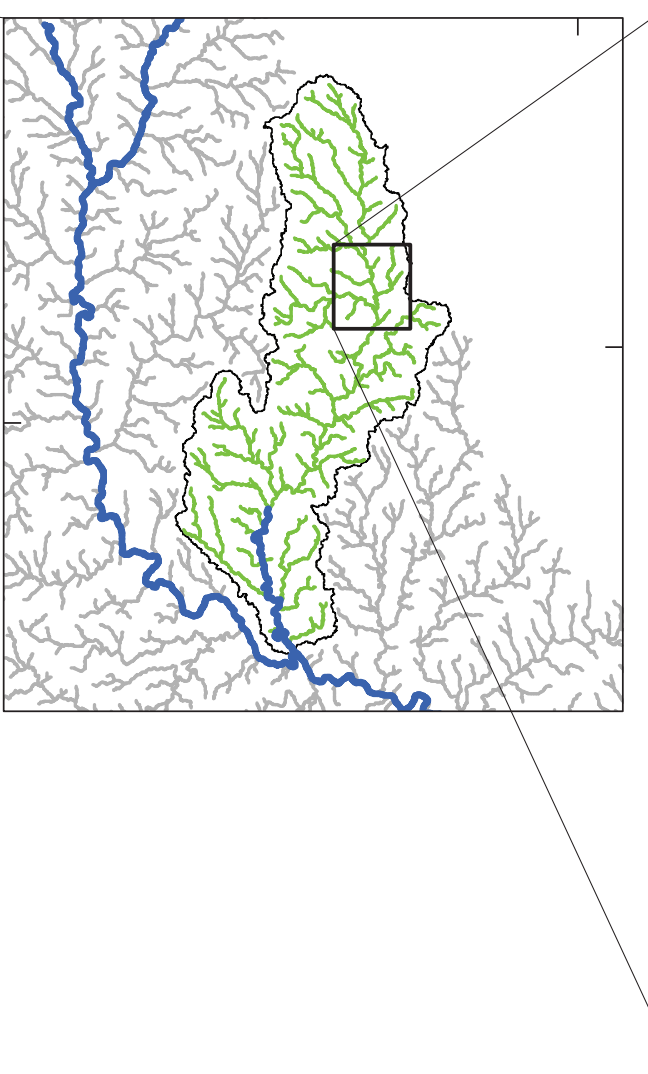

EXPLANATION

Coarse-scale model network hydrologic response unit

\section{Coarse-scale assessment hydrologic modeling} stream network

\section{Fine-scale model subbasi}

Upper Chattahoochee River Basin

Chestatee River Basin

Potato Creek Basin

Ichawaynochaway Creek Basin

Spring Creek Basin

Chipola River Ba

Upper Roanoke River Basin

Fine-scale Apalachicola-Chattahoochee-Flint River Basin network

Reach-based fine-scale network

valley floor width

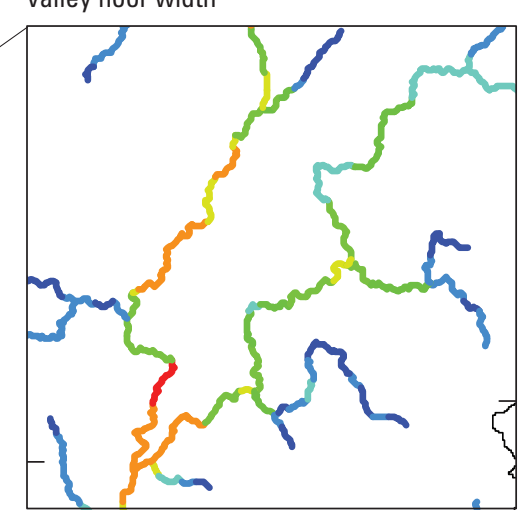

Segment-based fine-scale network valley floor width

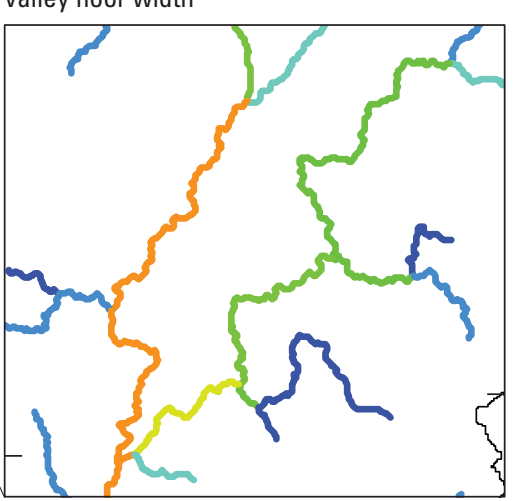

Potato Creek fine-scale model network example attribute Modeled valley floor width, in meters

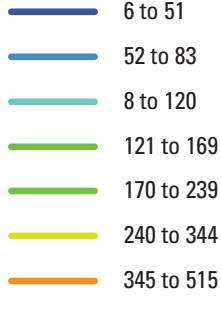

516 to 857

Figure 2. Coarse- and fine-scale stream networks for the basin, segment, and reach scales for hydrologic and aquatic biota models in the ApalachicolaChattahoochee-Flint River

Basin and the Upper

Roanoke River Basin.

Examples of reach-based and segment-based attribution with valley floor width are shown for the

Potato Creek Basin finescale network. 


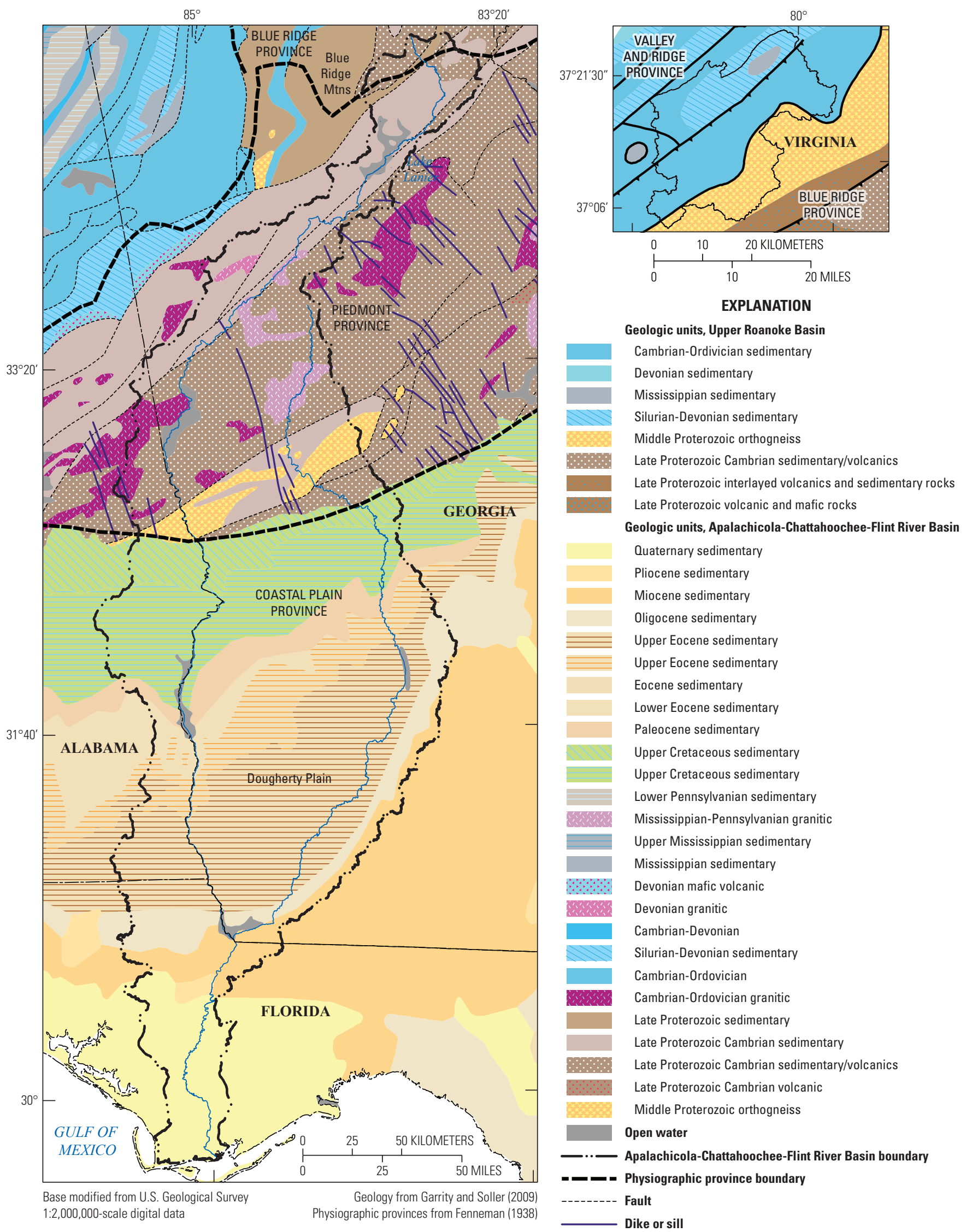

Figure 3. Regional geology of the Apalachicola-Chattahoochee-Flint River Basin in Georgia, Alabama, and Florida, and the Upper Roanoke River Basin in Virginia. 
downstream from Lake Blackshear flows through the Eocene Ocala and Suwannee Limestone in the Dougherty Plain (Ward and others, 2005) and is constrained by bedrock and has steeper gradients than the immediately upstream section. Rock shoals, snags, and boulders were removed on the Chattahoochee and Flint Rivers for steamboat navigation in the late 1800s and early 1900s (U.S. Congress, 1874, 1910; Freeman and others, 2008).

The Apalachicola River is formed by the junction of the Chattahoochee and Flint Rivers in the current (2013) location of Lake Seminole, which was impounded in 1957. Downstream from Lake Seminole and the Jim Woodruff Lock and Dam structure, the Apalachicola River is channelized and flows towards the Gulf of Mexico through a wide flood plain with backwater swamps. The Apalachicola River is highly engineered and was dredged from 1874 to 1956 to support navigation. In subsequent years, river-training structures and cutoffs were constructed and rocks were removed from the river channel in the upper reaches of the river (Light and others, 1998).

Upland land-use history in the southeastern United States has been implicated in stream disturbances since European settlement. In particular, accelerated soil erosion from historical farming practices has been identified as the cause of excessive sedimentation of flood plains and filling of impoundments (Trimble, 1974; Jacobson and Coleman, 1986; Jackson and others, 2005; Walter and Merritts, 2008). Following the effects of agricultural land uses, urbanization and increases in impervious area also have had the potential to alter runoff and sediment supply in many southeastern streams (Paul and Meyer, 2001; Riley and Jacobson, 2009; Carlisle and others, 2010). This history indicates that current (2013) stream conditions may be a product of historical or ongoing disturbances.

\section{Fine-Scale Subbasins}

In the multiscale model approach, six subbasins in the Apalachicola-Chattahoochee-Flint River Basin were chosen for hydrologic and fine-scale metapopulation models. This approach allows for cross-comparison of the effects of hydrology and climate change in basins ranging in size from $390-2,750 \mathrm{~km}^{2}$ with varying topography, degrees of urbanization, and dominant land use, building on a prototype model developed for part of the Potato Creek Basin in the Flint River drainage (Freeman and others, 2012). A seventh fine-scale model subbasin, the Upper Roanoke River Basin in the Albemarle-Pamlico Basin in the Valley and Ridge and Blue Ridge provinces in Virginia (figs. 1 and 2) was added to the modeling effort as part of a joint project with the U.S. Forest Service. The Upper Roanoke River Basin was intended to complement the SERAP modeling effort and provide a comparison to the model developed in the SERAP project for the Upper Chattahoochee Basin to address the potential temperature effects of climate change on coldwater biota in the southeastern United States, particularly eastern brook trout (Salvelinus fontinalis).
Two of the fine-scale model subbasins, the Upper Chattahoochee River and Chestatee River Basins (fig. 4), are located in the Blue Ridge and Piedmont physiographic provinces. The upstream parts of these basins largely are undeveloped and their streams flow through high-relief terrain. These two basins each have about $1,000 \mathrm{~m}$ of total relief and include some of the highest-gradient streams in the ApalachicolaChattahoochee-Flint River Basin. The Upper Roanoke River Basin is located in the Blue Ridge and Valley and Ridge provinces in Virginia. The Upper Roanoke River Basin has similar total basin relief to the Upper Chattahoochee River and Chestatee River Basins (923 m) and includes stream reaches with high gradients draining steep terrain, and lower-gradient streams draining the valleys (fig. 4).

The Potato Creek Basin is located in the Piedmont within the Flint River drainage basin. Potato Creek has a moderate amount of total basin relief, $272 \mathrm{~m}$ (fig. 4). A highresolution (1-m) Light Detection and Ranging (lidar) digital elevation model (DEM) dataset was available for the Potato Creek Basin and allowed for validation of regional hydraulic geometry relations and the statistical stream classification. The lidar dataset also allowed for an assessment of how well the 30-m DEM-derived stream network compared to on-the-ground features.

The Ichawaynochaway Creek, Spring Creek, and Chipola River Basins are in the Coastal Plain province and are generally low in relief, with total basin relief ranging from 95-148 m (fig. 5). The Ichawaynochaway Creek, Spring Creek, and the Chipola River Basins all contain karst topography in some or all of their drainage and generally are dominated by groundwater discharge (Couch and others, 1996). Water withdrawals for irrigation also are an important management issue in these basins.

\section{Approach, Methods, and Results}

Conventional datasets available in 2010-11 were used to develop a database of physical-habitat information for the stream network and classification. For instance, the National Elevation Dataset (NED) uses a variety of sources for elevation data and is updated as higher-resolution elevation information becomes available. The same base DEM-derived stream network is used at different scales for the coarse- and fine-scale PRMS hydrologic models, stream-temperature models, and species occupancy models within the SERAP project. There were iterative phases of network creation to arrive at a scale deemed appropriate for multiple uses. Most physical attributes were generated for the entire network and include multiple measures of stream size, mean reach gradient, and modeled valley width (table 1; appendix table 1-1).

The resultant stream networks were aggregated and attributed using two different criteria (segment- and reachbased) to accommodate hydrologic modeling and classification objectives. In addition, stream-network characteristics 

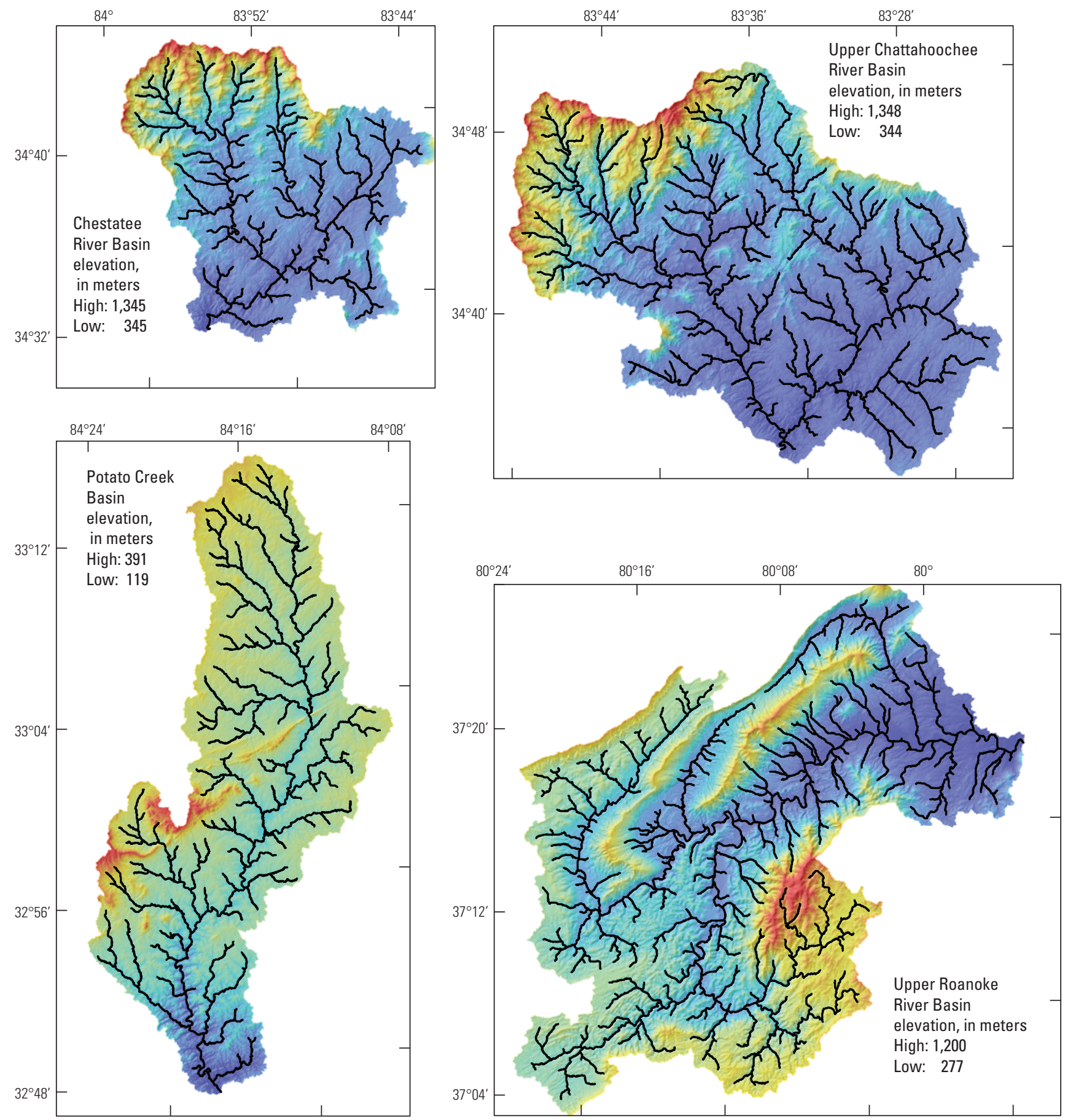

Base from U.S. Geological Survey National Elevation Data, 30-meter Digital Elevation Model Elevation is above North Americal Vertical Datum of 1988, in meters

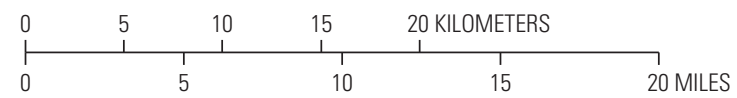

\section{EXPLANATION}

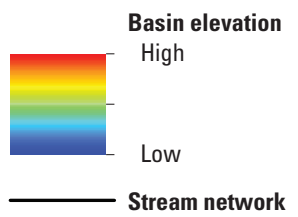

Figure 4. The digital elevation model (DEM) derived stream network for the Upper Chattahoochee River, Chestatee River, Potato Creek, and Upper Roanoke River Basins. 

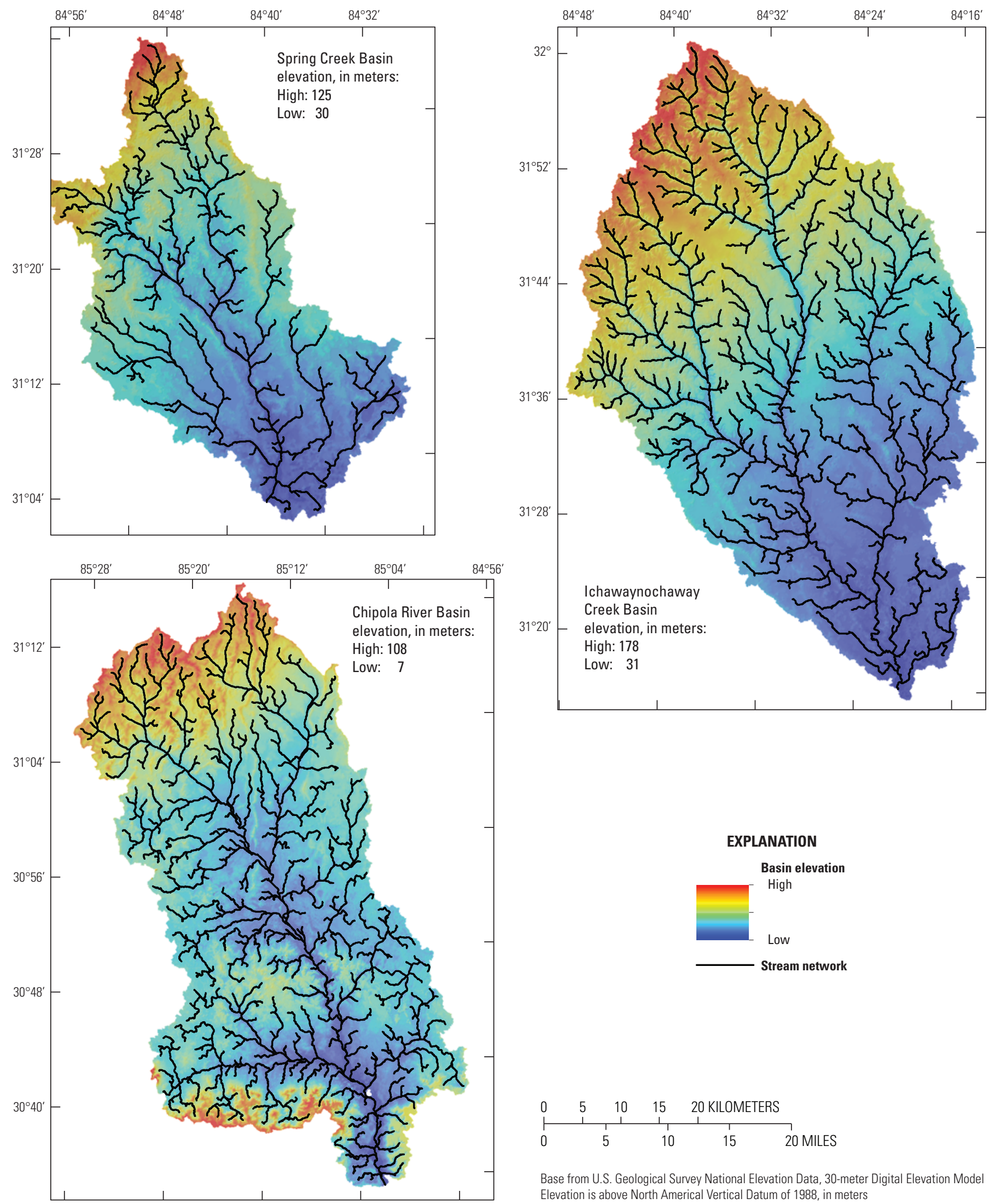

Base from U.S. Geological Survey National Elevation Data, 30-meter Digital Elevation Model Elevation is above North Americal Vertical Datum of 1988, in meters

Figure 5. The digital elevation model (DEM) derived stream network for the Spring Creek, Ichawaynochaway Creek, and Chipola River Basins. 
were aggregated and summarized for subbasins within the Apalachicola-Chattahoochee-Flint River Basin for application in the coarse-scale species-response model.

\section{Field Site Characterization}

In the fall of 2010, a field crew visited 59 stream sites within the DEM-based stream network in the ApalachicolaChattahoochee-Flint River Basin. Sites were selected before the statistical classification and were either on public land or near USGS streamgages with easy access. Streams were chosen throughout the Apalachicola-Chattahoochee-Flint River Basin and ranged in size from 1st order to 8th order streams. A two-person crew took measurements and made visual assessments over a reach approximately 10 times the bankfull channel width in length. A laser rangefinder was used to measure bankfull or active channel width, valley width (where possible), and incision from bankfull height. Over the entire reach, visual assessments were made of average channel pattern (straight, meandering, braided), presence of dikes or other channel-training structures, evidence of bank instability, and presence of large woody debris. Site specific characteristics were taken in a riffle, where present, or in the middle of the reach if no riffle was present. Site specific characteristics included dominant substrate (bedrock, boulder, cobble, gravel, and fines), bank material on the right and left bank, percent fines, and percent canopy cover. Photographs were taken using a video camera of a 360-degree view of the stream, riparian zone, and representative bed material. Field data and GISderived habitat and classification data for the 59 sites can be seen in tables 2 and 3.

\section{Stream Network Delineation and Attributes}

Two DEM analysis programs, Bldgrds and Netrace (Miller, 2008), were used to create the stream network and estimate various stream-channel and valley attributes for reaches in an Esri shapefile format (Esri, Redlands, California). Bldgrds and Netrace have adjustable parameters that were calibrated for the southeastern United States and intended use of the stream-network dataset. The 1/3 arcsecond (approximately 30-m) NED (Gesch and others, 2002; Gesch, 2007) DEM for the Apalachicola-Chattahoochee-Flint Basin was resampled to $30 \mathrm{~m}$ and converted to a floating point ASCII file. Netrace combines the flow direction, accumulation, and other information calculated by Bldgrds with the DEM using standard methods (Jenson and Domingue, 1988) to trace a stream network, and then assigns additional channel and valley attributes to individual reaches in the stream network (Miller, 2008). All attributes are populated to the stream-network shapefile.
The threshold value used to detect channel initiation was set based on subjective assessments of whether the output network was sufficiently detailed for stream classification and ecological modeling purposes in the diverse landscapes of the study area.

Netrace uses a slope-dependent drainage area threshold:

$$
a_{c r} \mathrm{~S}^{\alpha}=C
$$

where

$$
\begin{gathered}
a_{c r} \quad \begin{array}{l}
\text { is specific drainage area (defined as upslope } \\
\text { area per unit contour length) required for } \\
\text { channel initiation, }
\end{array} \\
S \quad \text { is surface gradient, } \\
\alpha \quad \text { is an exponent that varies between } 1 \text { and 2, } \\
\text { and } \\
\text { is a constant (Montgomery and Dietrich, } \\
\text { 1992; Dietrich and others, 1993). }
\end{gathered}
$$

Networks with a specific drainage area value of $100 \mathrm{~m}$ to $10,000 \mathrm{~m}$ were created for assessing the appropriate scale for the base network SERAP aquatics project fine models. Although a specific drainage area of $100 \mathrm{~m}$ was used for highand low-gradient areas in the Apalachicola-ChattahoocheeFlint River Basin for the stream network, this created a network that was too dense for hydrologic modeling in highgradient areas such as the Blue Ridge Mountains; however, greater values of specific drainage area created networks that were too sparse in the Coastal Plain for modeling the occupancy of aquatic species. As a result, the drainage network with a specific drainage area of $100 \mathrm{~m}$ was pared down by selecting and deleting first order segments with drainage basin areas less than $1 \mathrm{~km}^{2}$ or less than 1 kilometer $(\mathrm{km})$ in length.

The resultant stream network is different than the National Hydrography Dataset (NHD) stream networks (fig. 6). The DEM-derived network does not capture the low drainage density present in the karst region of the Dougherty Plain well, where subsurface flow is important. The DEMderived model network is less dense than the high-resolution (1:24,000 scale) NHD, but similar in drainage density and structure in most areas to the medium-resolution $(1: 100,000$ scale) NHD.

Drainage basin area for each stream segment was defined as the land surface area with runoff that eventually passes through the downstream end of the segment. This number is cumulative and includes the drainage all the way to the upstream-most drainage divide. The drainage areas associated with segments in the final fine-scale network ranged from $1-49,735 \mathrm{~km}^{2}$ (fig. 7). Mean drainage basin area was $371 \mathrm{~km}^{2}$ (table 1). 
Table 1. Geomorphic variables and basin summary data for the Apalachicola-Chattahoochee-Flint River Basin and six fine-scale model basins nested within the ApalachicolaChattahoochee-Flint River Basin as well as the Upper Roanoke River Basin in Virginia.

[--, no data available at this scale

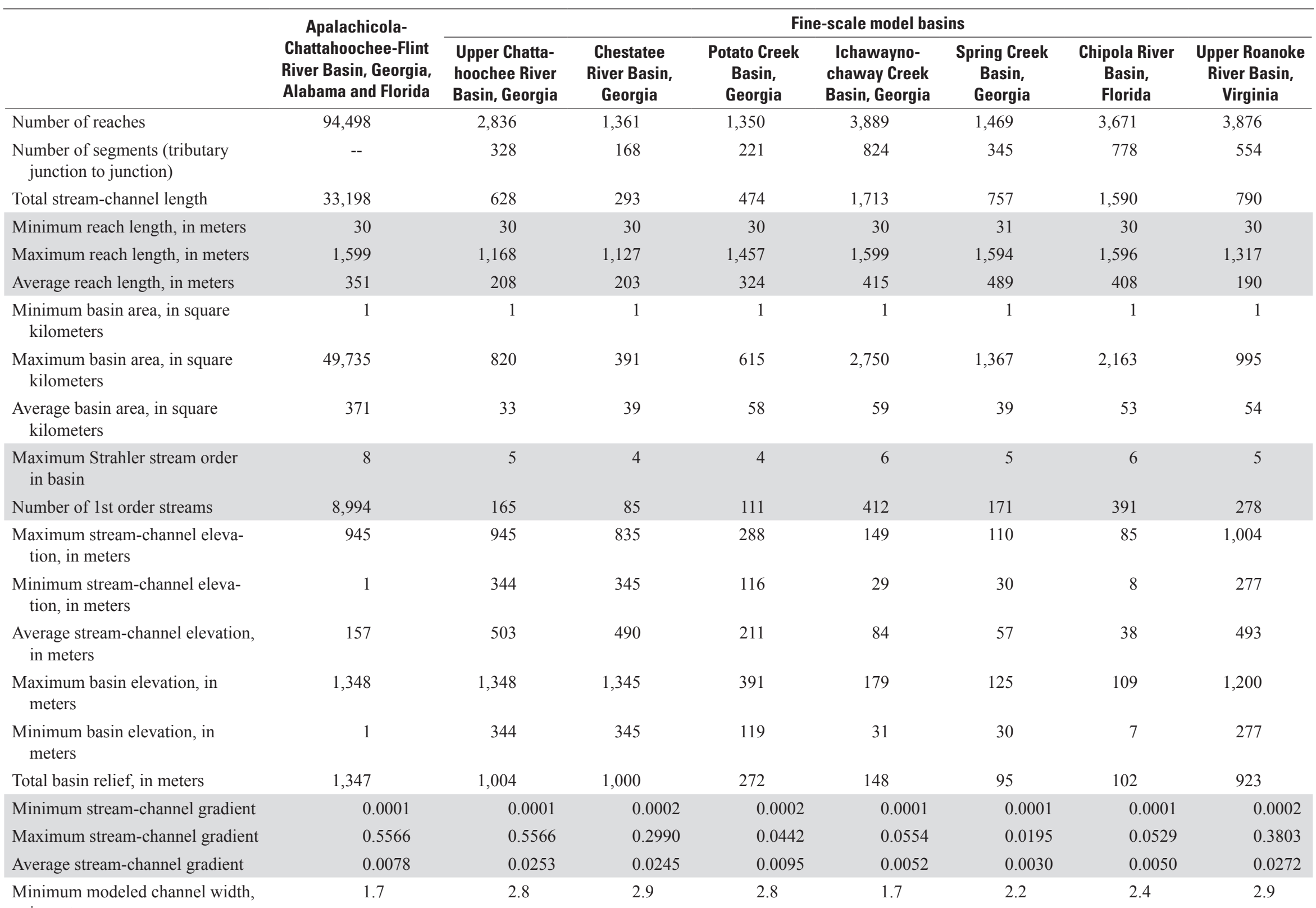


Table 1. Geomorphic variables and basin summary data for the Apalachicola-Chattahoochee-Flint River Basin and six fine-scale model basins nested within the Apalachicola-

Chattahoochee-Flint River Basin as well as the Upper Roanoke River Basin in Virginia._Continued

[--, no data available at this scale $]$

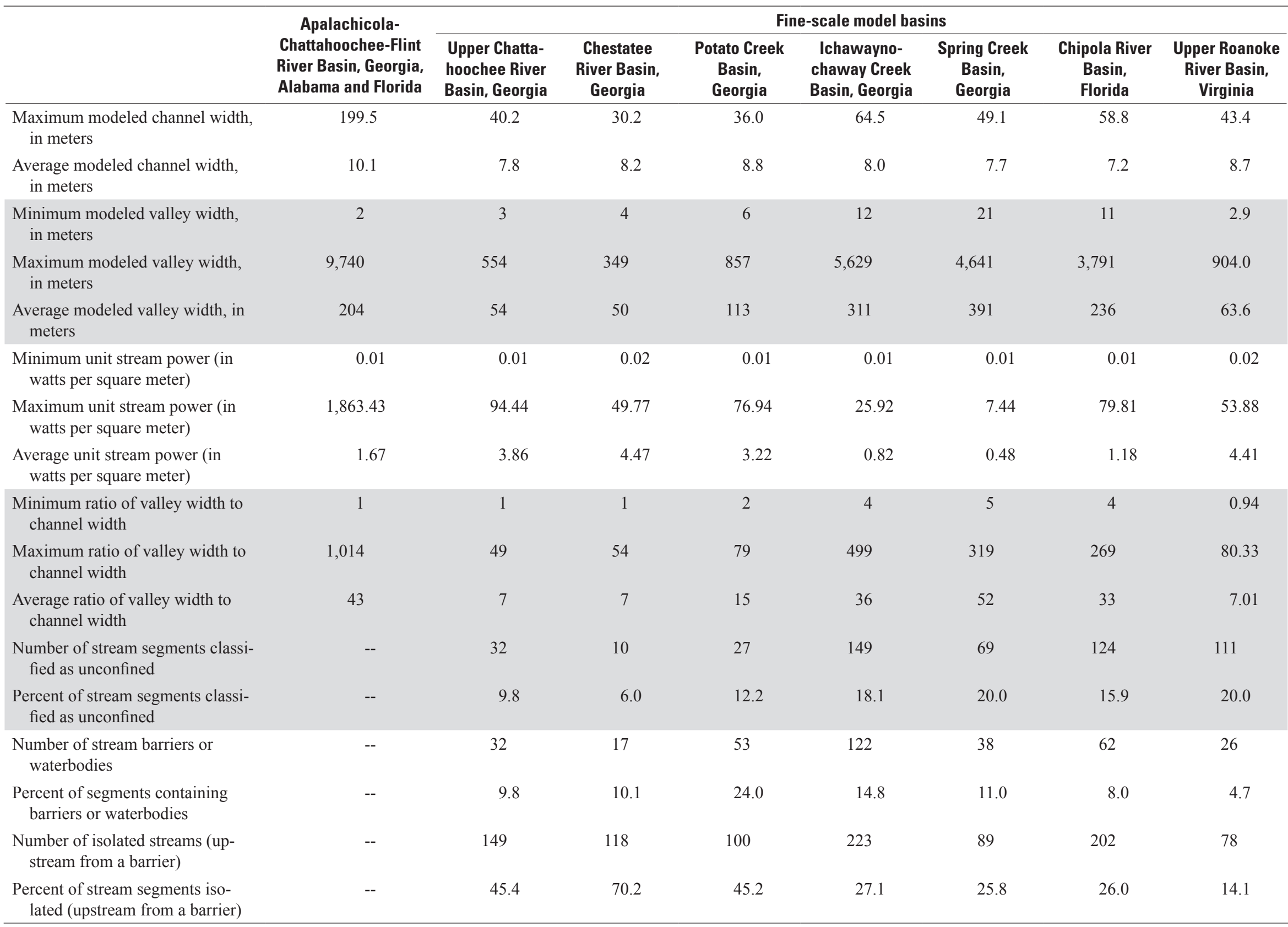


Table 2. Field measurements of selected geomorphic and habitat variables for 59 streams in the Apalachicola-Chattahoochee-Flint River Basin.

$[--$, no data available $]$

\begin{tabular}{|c|c|c|c|c|c|c|c|c|c|}
\hline \multirow{2}{*}{$\begin{array}{c}\text { Name } \\
\text { (from National } \\
\text { Hydrography Dataset) }\end{array}$} & \multicolumn{9}{|c|}{ Field data } \\
\hline & $\begin{array}{l}\text { Bankfull width, } \\
\text { in meters }\end{array}$ & $\begin{array}{l}\text { Valley width, } \\
\text { in meters }\end{array}$ & $\begin{array}{l}\text { Bank height, } \\
\text { in meters }\end{array}$ & $\begin{array}{c}\text { Dominant } \\
\text { channel pattern }\end{array}$ & $\begin{array}{l}\text { Dominant } \\
\text { substrate }\end{array}$ & $\begin{array}{c}\text { Presence of } \\
\text { bedrock }\end{array}$ & $\begin{array}{l}\text { Approximate } \\
\text { percent fines }\end{array}$ & $\begin{array}{c}\text { Right bank } \\
\text { material }\end{array}$ & $\begin{array}{c}\text { Left bank } \\
\text { material }\end{array}$ \\
\hline Smith Creek & 9.5 & 12 & 1.0 & straight & boulder & not present & 50 & boulder & boulder. \\
\hline unnamed & 4.5 & 9 & 2.0 & straight & cobble & bedrock present & 80 & & bedrock, boulder. \\
\hline Spolicane Creek & 9.9 & 75 & 0.5 & straight & cobble & not present & 50 & fines & boulder, fines. \\
\hline Chattahoochee River & 25 & 35 & 0.5 & meandering & cobble & not present & 10 & fines & fines. \\
\hline Horton Creek & 8.2 & 14.8 & 2.0 & straight & bedrock & bedrock present & 10 & bedrock & bedrock. \\
\hline Dukes Creek & 11 & 100 & 0.9 & straight & boulder & bedrock present & 30 & bedrock & bedrock. \\
\hline Ash Creek & 6 & -- & 0.5 & meandering & cobble & not present & 50 & fines & boulder, fines. \\
\hline unnamed & 6 & -- & 0.6 & straight & cobble & not present & 80 & fines & fines. \\
\hline Waters Creek & 11 & -- & 0.0 & straight & bedrock & bedrock present & 10 & bedrock & bedrock. \\
\hline unnamed & 9 & -- & 0.7 & straight & boulder & not present & 30 & boulder & boulder. \\
\hline Frogtown Creek & 10 & -- & 0.5 & meandering & cobble & bedrock present & 30 & bedrock & bedrock. \\
\hline Chestatee River & 11.5 & -- & 0.0 & straight & cobble & bedrock present & 50 & bedrock & bedrock. \\
\hline Boggs Creek & 10 & 12 & 0.0 & straight & bedrock & bedrock present & 30 & bedrock & bedrock. \\
\hline Chestatee River & 26 & -- & 2.0 & meandering & boulder & not present & 80 & boulder & boulder. \\
\hline Chattahoochee River & 87 & -- & 0.0 & straight & fines & not present & 100 & fines & fines. \\
\hline Big Creek & 17 & -- & 1.7 & straight & bedrock & bedrock present & 80 & fines & bedrock. \\
\hline Sweetwater Creek & 20 & -- & 2.4 & meandering & cobble & bedrock present & 80 & bedrock & fines. \\
\hline Sweetwater Creek & 33 & -- & 1.8 & meandering & fines & not present & 80 & fines & fines. \\
\hline Little Bear Creek & 7.5 & -- & 1.9 & meandering & gravel & not present & 90 & fines & fines. \\
\hline Bear Creek & 9 & -- & 1.8 & meandering & gravel & not present & 90 & fines & fines. \\
\hline unnamed & 5 & -- & 1.5 & meandering & gravel & not present & 90 & fines & fines. \\
\hline Cedar Branch & 6.7 & -- & 1.3 & meandering & gravel & not present & 90 & fines & fines. \\
\hline unnamed & 3.2 & -- & 0.9 & meandering & gravel & not present & 50 & fines & fines. \\
\hline unnamed & 2.5 & -- & 0.0 & meandering & cobble & not present & 50 & fines & fines. \\
\hline Bethel Creek & 1.5 & 4 & 0.0 & straight & fines & not present & 100 & fines & fines. \\
\hline Standing Boy Creek & 15.5 & -- & 0.8 & meandering & fines & not present & 90 & fines & fines. \\
\hline Hannahatchee Creek & 9.3 & -- & 0.6 & meandering & fines & not present & 100 & fines & fines. \\
\hline unnamed & 4 & -- & 2.7 & straight & fines & not present & 100 & fines & fines. \\
\hline Bussey Branch & 5.3 & -- & 2.3 & straight & fines & not present & 100 & fines & fines. \\
\hline Turner Creek & 6.2 & 50 & 40.0 & braided & fines & not present & 100 & fines & fines. \\
\hline
\end{tabular}


Table 2. Field measurements of selected geomorphic and habitat variables for 59 streams in the Apalachicola-Chattahoochee-Flint River Basin.-Continued

[--, no data available]

\begin{tabular}{|c|c|c|c|c|c|c|c|c|c|}
\hline \multirow{2}{*}{$\begin{array}{c}\text { Name } \\
\text { (from National } \\
\text { Hydrography Dataset) }\end{array}$} & \multicolumn{9}{|c|}{ Field data } \\
\hline & $\begin{array}{l}\text { Bankfull width, } \\
\text { in meters }\end{array}$ & $\begin{array}{l}\text { Valley width, } \\
\text { in meters }\end{array}$ & $\begin{array}{l}\text { Bank height, } \\
\text { in meters }\end{array}$ & $\begin{array}{c}\text { Dominant } \\
\text { channel pattern }\end{array}$ & $\begin{array}{l}\text { Dominant } \\
\text { substrate }\end{array}$ & $\begin{array}{l}\text { Presence of } \\
\text { bedrock }\end{array}$ & $\begin{array}{l}\text { Approximate } \\
\text { percent fines }\end{array}$ & $\begin{array}{c}\text { Right bank } \\
\text { material }\end{array}$ & $\begin{array}{l}\text { Left bank } \\
\text { material }\end{array}$ \\
\hline South Fork Cowikee Creek & 23 & -- & 4.6 & meandering & fines & bedrock present & 80 & fines & bedrock, fines. \\
\hline Pataula Creek & 20 & -- & 0.0 & straight & fines & not present & 100 & fines & fines. \\
\hline Chatahoochee River & 116 & -- & 0.0 & straight & bedrock & bedrock present & 80 & bedrock, fines & bedrock, fines. \\
\hline Flat Creek & 7 & -- & 0.0 & meandering & fines & not present & 50 & fines & fines. \\
\hline unnamed & 2.5 & -- & 0.0 & meandering & fines & not present & 100 & fines & fines. \\
\hline Flint River & 43 & -- & 1.1 & straight & fines & bedrock present & 100 & fines & fines. \\
\hline White Oak Creek & 24 & -- & 1.1 & meandering & fines & not present & 100 & fines & fines. \\
\hline unnamed & 0 & -- & 0.0 & meandering & fines & not present & 90 & gravel, fines & gravel, fines. \\
\hline Flint River & 85 & -- & 2.9 & meandering & cobble & bedrock present & 10 & fines & fines. \\
\hline unnamed & 6.6 & -- & 0.7 & meandering & cobble & bedrock present & 50 & fines & fines. \\
\hline Whitewater Creek & 14 & -- & 0.0 & meandering & fines & not present & 100 & fines & fines. \\
\hline Flint River & 66 & -- & 0.9 & meandering & fines & not present & 90 & fines & fines. \\
\hline Flint River & 70 & -- & 0.8 & meandering & fines & not present & 100 & fines & fines. \\
\hline unnamed & 0 & -- & 0.0 & straight & fines & not present & 100 & fines & fines. \\
\hline Flint River & 90 & -- & 5.5 & meandering & bedrock & bedrock present & 80 & bedrock, fines & bedrock, fines. \\
\hline $\begin{array}{l}\text { West Chickasawhatchee } \\
\text { Creek }\end{array}$ & 11 & -- & 0.0 & meandering & fines & not present & 100 & fines & fines. \\
\hline Kiokee Creek & 5 & -- & 0.0 & wetland & fines & not present & 100 & fines & fines. \\
\hline Keel Creek & 6.5 & -- & 0.0 & straight & fines & bedrock present & 100 & fines & fines. \\
\hline Ichawaynochaway Creek & 35 & -- & 1.1 & straight & cobble & bedrock present & 80 & bedrock, fines & bedrock, fines. \\
\hline Ichawaynochaway Creek & 33 & -- & 0.0 & meandering & boulder & bedrock present & 80 & bedrock, fines & bedrock, fines. \\
\hline Big Cypress Creek & 8 & -- & 0.2 & meandering & bedrock & bedrock present & 80 & bedrock, fines & bedrock, fines. \\
\hline Rock Creek & 10 & -- & 1.1 & meandering & fines & not present & 90 & fines & fines. \\
\hline Apalachicola River & 260 & -- & 1.8 & meandering & fines & not present & 100 & boulder, fines & fines. \\
\hline Marshall Creek & 5 & -- & 0.0 & braided & fines & not present & 100 & fines & fines. \\
\hline Spring Branch & 18 & -- & 0.0 & straight & fines & not present & 100 & fines & fines. \\
\hline Chipola River & 27 & -- & 0.0 & meandering & fines & bedrock & 100 & fines & fines. \\
\hline Muddy Branch & 10 & -- & 0.0 & $\begin{array}{l}\text { meandering/ } \\
\text { braided }\end{array}$ & fines & not present & 100 & fines & fines. \\
\hline Chipola River & 26 & -- & 0.6 & meandering & bedrock & bedrock & 20 & bedrock, fines & fines. \\
\hline Chipola River & 75 & -- & 0.0 & braided & fines & not present & 100 & fines & fines. \\
\hline
\end{tabular}


Table 3. Geographic information system (GIS) derived estimates of selected geomorphic variables for 59 streams in the Apalachicola-Chattahoochee-Flint River Basin, based on 30-meter digital elevation model (DEM) data, and channel classification assignments based on 6- and 12-cluster classification models.

[Confined column: 0, unconfined channel; 1, confined channel. Confined channels are defined as channels either less than 10 meters wide or with a ratio of valley width to modeled channel width less than 4]

\begin{tabular}{|c|c|c|c|c|c|c|c|c|c|c|c|c|c|}
\hline \multirow[b]{2}{*}{$\begin{array}{c}\text { Name } \\
\text { (from National } \\
\text { Hydrologic Dataset) }\end{array}$} & \multicolumn{13}{|c|}{ GIS data } \\
\hline & $\begin{array}{c}\text { Area, } \\
\text { in square } \\
\text { kilometers }\end{array}$ & $\begin{array}{l}\text { Elevation, } \\
\text { in meters }\end{array}$ & $\begin{array}{c}\text { Mean reach } \\
\text { gradient, } \\
\text { meters per } \\
\text { meter }\end{array}$ & $\begin{array}{l}\text { Channel } \\
\text { width, } \\
\text { in meters }\end{array}$ & $\begin{array}{c}\text { Valley } \\
\text { width, } \\
\text { in meters }\end{array}$ & $\begin{array}{l}\text { Strahler } \\
\text { stream } \\
\text { order }\end{array}$ & $\begin{array}{c}\text { Shreve } \\
\text { stream } \\
\text { order }\end{array}$ & $\begin{array}{l}\text { Unit stream } \\
\text { power, } \\
\text { in watts } \\
\text { per meter }\end{array}$ & Sinuosity & $\begin{array}{c}\text { Ratio of } \\
\text { valley width } \\
\text { to channel } \\
\text { width }\end{array}$ & Confined & $\begin{array}{l}\text { 6-cluster } \\
\text { class }\end{array}$ & $\begin{array}{l}\text { 12-cluster } \\
\text { class }\end{array}$ \\
\hline Smith Creek & 16.4 & 499.2 & 0.0256 & 8.7 & 14.7 & 2 & 3 & 6.87 & 1.32 & 1.7 & 1 & 1 & 11 \\
\hline unnamed & 2.4 & 503.3 & 0.0495 & 4.1 & 25.5 & 1 & 1 & 4.18 & 1.19 & 6.2 & 1 & 1 & 11 \\
\hline Spolicane Creek & 3.4 & 270.9 & 0.0036 & 4.7 & 80.1 & 1 & 1 & 0.38 & 1.30 & 17.0 & 1 & 1 & 5 \\
\hline Chattahoochee River & 110.1 & 438 & 0.0063 & 18.4 & 67.2 & 3 & 25 & 5.26 & 1.34 & 3.7 & 1 & 1 & 1 \\
\hline Horton Creek & 3.7 & 438.6 & 0.046 & 4.9 & 21.5 & 1 & 1 & 5.00 & 1.34 & 4.4 & 1 & 1 & 11 \\
\hline Dukes Creek & 31.7 & 470.2 & 0.0161 & 11.3 & 80.4 & 3 & 9 & 6.40 & 1.28 & 7.1 & 0 & 1 & 11 \\
\hline Ash Creek & 1.3 & 519.3 & 0.0223 & 3.3 & 30.5 & 1 & 1 & 1.33 & 1.41 & 9.2 & 1 & 1 & 11 \\
\hline unnamed & 1.6 & 342.2 & 0.0126 & 3.5 & 38.7 & 1 & 1 & 0.86 & 1.41 & 11.1 & 1 & 1 & 11 \\
\hline Waters Creek & 13.8 & 515.3 & 0.0238 & 8.2 & 26.0 & 2 & 3 & 5.74 & 1.97 & 3.2 & 1 & 1 & 11 \\
\hline unnamed & 7.4 & 631.4 & 0.0276 & 6.4 & 51.4 & 2 & 3 & 4.61 & 1.26 & 8.0 & 1 & 1 & 11 \\
\hline Frogtown Creek & 9.7 & 611.6 & 0.0342 & 7.1 & 52.7 & 2 & 3 & 6.72 & 1.26 & 7.4 & 1 & 1 & 11 \\
\hline Chestatee River & 42.1 & 481.7 & 0.0088 & 12.6 & 53.9 & 3 & 10 & 4.15 & 1.97 & 4.3 & 1 & 1 & 1 \\
\hline Boggs Creek & 18.5 & 486.7 & 0.0295 & 9.2 & 35.2 & 2 & 4 & 8.45 & 1.34 & 3.8 & 1 & 1 & 11 \\
\hline Chestatee River & 390.3 & 346.3 & 0.0027 & 30.1 & 182.9 & 4 & 85 & 4.82 & 1.71 & 6.1 & 0 & 4 & 4 \\
\hline Chattahoochee River & $3,183.8$ & 260.8 & 0.0011 & 68.3 & 355.8 & 5 & 617 & 6.90 & 1.56 & 5.2 & 0 & 3 & 3 \\
\hline Big Creek & 266.6 & 283.2 & 0.0094 & 26.0 & 98.3 & 4 & 58 & 13.33 & 2.33 & 3.8 & 1 & 4 & 4 \\
\hline Sweetwater Creek & 682.3 & 225.6 & 0.0002 & 37.5 & 258.0 & 5 & 110 & 0.50 & 1.31 & 6.9 & 0 & 4 & 4 \\
\hline Sweetwater Creek & 683.3 & 225.6 & 0.0002 & 37.5 & 689.6 & 5 & 110 & 0.50 & 1.31 & 18.4 & 0 & 4 & 4 \\
\hline Little Bear Creek & 17.0 & 239.6 & 0.0111 & 8.9 & 108.9 & 2 & 3 & 3.03 & 1.15 & 12.2 & 1 & 1 & 5 \\
\hline Bear Creek & 55.3 & 237.8 & 0.0005 & 14.1 & 438.8 & 3 & 12 & 0.28 & 1.07 & 31.1 & 0 & 1 & 1 \\
\hline unnamed & 7.6 & 241 & 0.0037 & 6.5 & 88.3 & 2 & 2 & 0.63 & 1.20 & 13.6 & 1 & 1 & 5 \\
\hline Cedar Branch & 8.0 & 239.4 & 0.0049 & 6.6 & 90.7 & 2 & 2 & 0.86 & 1.15 & 13.7 & 1 & 1 & 5 \\
\hline unnamed & 1.1 & 206.5 & 0.003 & 3.0 & 75.6 & 1 & 1 & 0.17 & 1.20 & 25.2 & 1 & 1 & 5 \\
\hline unnamed & 1.1 & 263.3 & 0.0179 & 3.0 & 25.5 & 1 & 1 & 0.98 & 1.21 & 8.5 & 1 & 1 & 11 \\
\hline Bethel Creek & 1.1 & 312.9 & 0.0257 & 3.0 & 13.3 & 1 & 1 & 1.35 & 1.16 & 4.4 & 1 & 1 & 11 \\
\hline Standing Boy Creek & 118.4 & 103.2 & 0.0002 & 18.9 & 262.1 & 4 & 21 & 0.17 & 1.38 & 13.9 & 0 & 4 & 1 \\
\hline Hannahatchee Creek & 49.2 & 103.5 & 0.0015 & 13.4 & 148.2 & 3 & 10 & 0.78 & 1.26 & 11.1 & 0 & 1 & 1 \\
\hline unnamed & 6.7 & 104.3 & 0.0013 & 6.1 & 83.1 & 2 & 2 & 0.21 & 1.19 & 13.6 & 1 & 1 & 5 \\
\hline Bussey Branch & 8.6 & 103.9 & 0.0081 & 6.8 & 78.0 & 1 & 1 & 1.48 & 1.20 & 11.5 & 1 & 1 & 5 \\
\hline Turner Creek & 2.3 & 107.8 & 0.0105 & 4.0 & 95.1 & 1 & 1 & 0.86 & 1.15 & 23.8 & 1 & 1 & 5 \\
\hline
\end{tabular}


Table 3. Geographic information system (GIS) derived estimates of selected geomorphic variables for 59 streams in the Apalachicola-Chattahoochee-Flint River Basin, based on 30-meter digital elevation model (DEM) data, and channel classification assignments based on 6- and 12-cluster classification models.-Continued

[Confined column: 0, unconfined channel; 1, confined channel. Confined channels are defined as channels either less than 10 meters wide or with a ratio of valley width to modeled channel width less than 4]

\begin{tabular}{|c|c|c|c|c|c|c|c|c|c|c|c|c|c|}
\hline \multirow[b]{2}{*}{$\begin{array}{c}\text { Name } \\
\text { (from National } \\
\text { Hydrologic Dataset) }\end{array}$} & \multicolumn{13}{|c|}{ GIS data } \\
\hline & $\begin{array}{c}\text { Area, } \\
\text { in square } \\
\text { kilometers }\end{array}$ & $\begin{array}{l}\text { Elevation, } \\
\text { in meters }\end{array}$ & $\begin{array}{l}\text { Mean reach } \\
\text { gradient, } \\
\text { meters per } \\
\text { meter }\end{array}$ & $\begin{array}{l}\text { Channel } \\
\text { width, } \\
\text { in meters }\end{array}$ & $\begin{array}{c}\text { Valley } \\
\text { width, } \\
\text { in meters }\end{array}$ & $\begin{array}{l}\text { Strahler } \\
\text { stream } \\
\text { order }\end{array}$ & $\begin{array}{l}\text { Shreve } \\
\text { stream } \\
\text { order }\end{array}$ & $\begin{array}{l}\text { Unit stream } \\
\text { power, } \\
\text { in watts } \\
\text { per meter }\end{array}$ & Sinuosity & $\begin{array}{l}\text { Ratio of } \\
\text { valley width } \\
\text { to channel } \\
\text { width }\end{array}$ & Confined & $\begin{array}{l}\text { 6-cluster } \\
\text { class }\end{array}$ & $\begin{array}{l}\text { 12-cluster } \\
\text { class }\end{array}$ \\
\hline $\begin{array}{l}\text { South Fork Cowikee } \\
\text { Creek }\end{array}$ & 69.0 & 59.5 & 0.0086 & 15.3 & 65.3 & 4 & 16 & 5.44 & 1.24 & 4.3 & 1 & 1 & 1 \\
\hline Pataula Creek & 176.9 & 88.1 & 0.0015 & 22.1 & 521.7 & 4 & 34 & 1.67 & 1.30 & 23.6 & 0 & 4 & 1 \\
\hline Chatahoochee River & $21,230.4$ & 23 & 0.0002 & 143.2 & $1,157.5$ & 7 & 4,208 & 3.91 & 1.11 & 8.1 & 0 & 2 & 2 \\
\hline Flat Creek & 50.9 & 230 & 0.0049 & 13.6 & 411.9 & 2 & 10 & 2.58 & 1.30 & 30.3 & 0 & 1 & 1 \\
\hline unnamed & 2.7 & 230 & 0.0046 & 4.3 & 158.2 & 1 & 1 & 0.41 & 1.30 & 36.8 & 1 & 1 & 5 \\
\hline Flint River & $1,936.5$ & 212.5 & 0.0004 & 56.3 & 792.0 & 6 & 342 & 1.86 & 1.11 & 14.1 & 0 & 3 & 3 \\
\hline White Oak Creek & 392.0 & 215.3 & 0.0026 & 30.2 & 582.3 & 4 & 71 & 4.65 & 1.25 & 19.3 & 0 & 4 & 4 \\
\hline unnamed & 9.3 & 213.5 & 0.0072 & 7.0 & 84.9 & 2 & 3 & 1.37 & 1.22 & 12.1 & 1 & 1 & 5 \\
\hline Flint River & $3,124.9$ & 169.9 & 0.0007 & 67.8 & 232.2 & 6 & 590 & 4.34 & 1.36 & 3.4 & 1 & 3 & 3 \\
\hline unnamed & 2.8 & 121.3 & 0.0152 & 4.3 & 76.4 & 1 & 1 & 1.42 & 1.26 & 17.8 & 1 & 1 & 11 \\
\hline Whitewater Creek & 181.4 & 111.3 & 0.0017 & 22.3 & 224.4 & 4 & 36 & 1.92 & 1.24 & 10.1 & 0 & 4 & 1 \\
\hline Flint River & $6,812.5$ & 82.1 & 0.0002 & 91.9 & $1,292.0$ & 6 & 1,278 & 1.98 & 1.17 & 14.1 & 0 & 3 & 3 \\
\hline Flint River & $8,237.9$ & 75.6 & 0.0002 & 99.0 & $2,356.6$ & 6 & 1,560 & 2.22 & 1.59 & 23.8 & 0 & 3 & 8 \\
\hline unnamed & 14.8 & 30.4 & 0.0171 & 8.4 & 257.5 & 3 & 4 & 4.29 & 1.27 & 30.7 & 1 & 1 & 11 \\
\hline Flint River & $14,988.8$ & 30.4 & 0.0002 & 125.0 & $2,250.7$ & 7 & 2,687 & 3.17 & 1.27 & 18.0 & 0 & 2 & 8 \\
\hline $\begin{array}{l}\text { West Chicka- } \\
\text { sawhatchee Creek }\end{array}$ & 394.0 & 56.4 & 0.0013 & 30.2 & $1,724.8$ & 4 & 60 & 2.33 & 1.25 & 57.1 & 0 & 4 & 6 \\
\hline Kiokee Creek & 149.2 & 55.9 & 0.0024 & 20.7 & $1,117.2$ & 4 & 18 & 2.41 & 1.35 & 54.0 & 0 & 4 & 1 \\
\hline Keel Creek & 48.6 & 51.9 & 0.0016 & 13.0 & $1,491.1$ & 2 & 4 & 0.84 & 1.22 & 114.7 & 0 & 4 & 6 \\
\hline $\begin{array}{l}\text { Ichawaynochaway } \\
\text { Creek }\end{array}$ & $2,570.5$ & 35 & 0.003 & 62.8 & 866.1 & 6 & 391 & 16.56 & 1.54 & 13.8 & 0 & 3 & 3 \\
\hline $\begin{array}{l}\text { Ichawaynochaway } \\
\text { Creek }\end{array}$ & $2,751.4$ & 29 & 0.0002 & 64.5 & 751.7 & 6 & 412 & 1.15 & 1.60 & 11.7 & 0 & 3 & 3 \\
\hline Big Cypress Creek & 166.9 & 32 & 0.0081 & 21.6 & 147.1 & 3 & 15 & 8.70 & 1.35 & 6.8 & 0 & 4 & 1 \\
\hline Rock Creek & 14.4 & 20 & 0.0114 & 8.3 & 59.6 & 2 & 6 & 2.83 & 1.36 & 7.2 & 1 & 1 & 5 \\
\hline Apalachicola River & $45,436.5$ & 9.8 & 0.0008 & 192.6 & 911.3 & 8 & 8,296 & 24.66 & 2.93 & 4.7 & 1 & 2 & 7 \\
\hline Marshall Creek & 483.5 & 27 & 0.0014 & 32.8 & $1,254.4$ & 4 & 82 & 2.83 & 1.12 & 38.2 & 0 & 4 & 4 \\
\hline Spring Branch & 50.3 & 27 & 0.0002 & 13.5 & 806.5 & 3 & 8 & 0.10 & 1.12 & 59.7 & 0 & 1 & 1 \\
\hline Chipola River & $1,239.8$ & 22 & 0.003 & 47.3 & 485.9 & 5 & 211 & 10.69 & 1.23 & 10.3 & 0 & 4 & 4 \\
\hline Muddy Branch & 1.2 & 55 & 0.0101 & 3.2 & 38.5 & 1 & 1 & 0.56 & 1.14 & 12.0 & 1 & 1 & 5 \\
\hline Chipola River & $1,362.6$ & 21 & 0.0046 & 49.1 & 511.3 & 5 & 232 & 17.33 & 1.33 & 10.4 & 0 & 4 & 4 \\
\hline Chipola River & $3,241.4$ & 1.4 & 0.0002 & 68.8 & $9,000.0$ & 6 & 568 & 1.27 & 1.09 & 130.8 & 0 & 6 & 10 \\
\hline
\end{tabular}



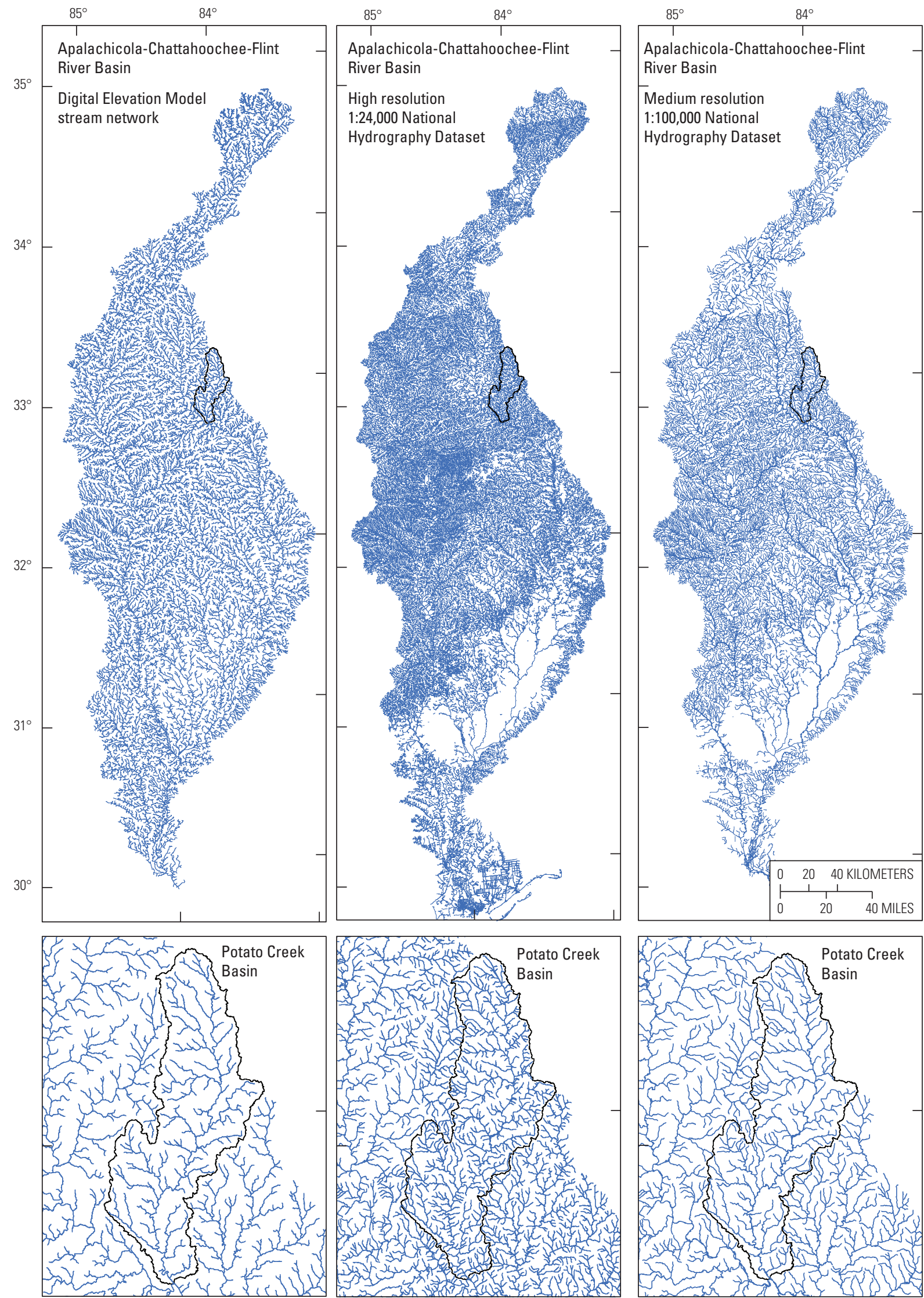

Figure 6. The digital elevation model (DEM) stream network and the high- and medium-resolution National Hydrography Datasets (NHD) for the Apalachicola-

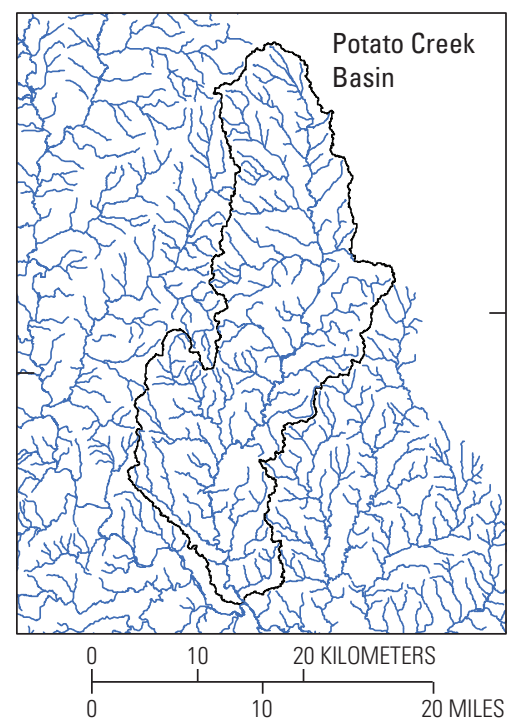
Chattahoochee-Flint River Basin and the Potato Creek Basin. 

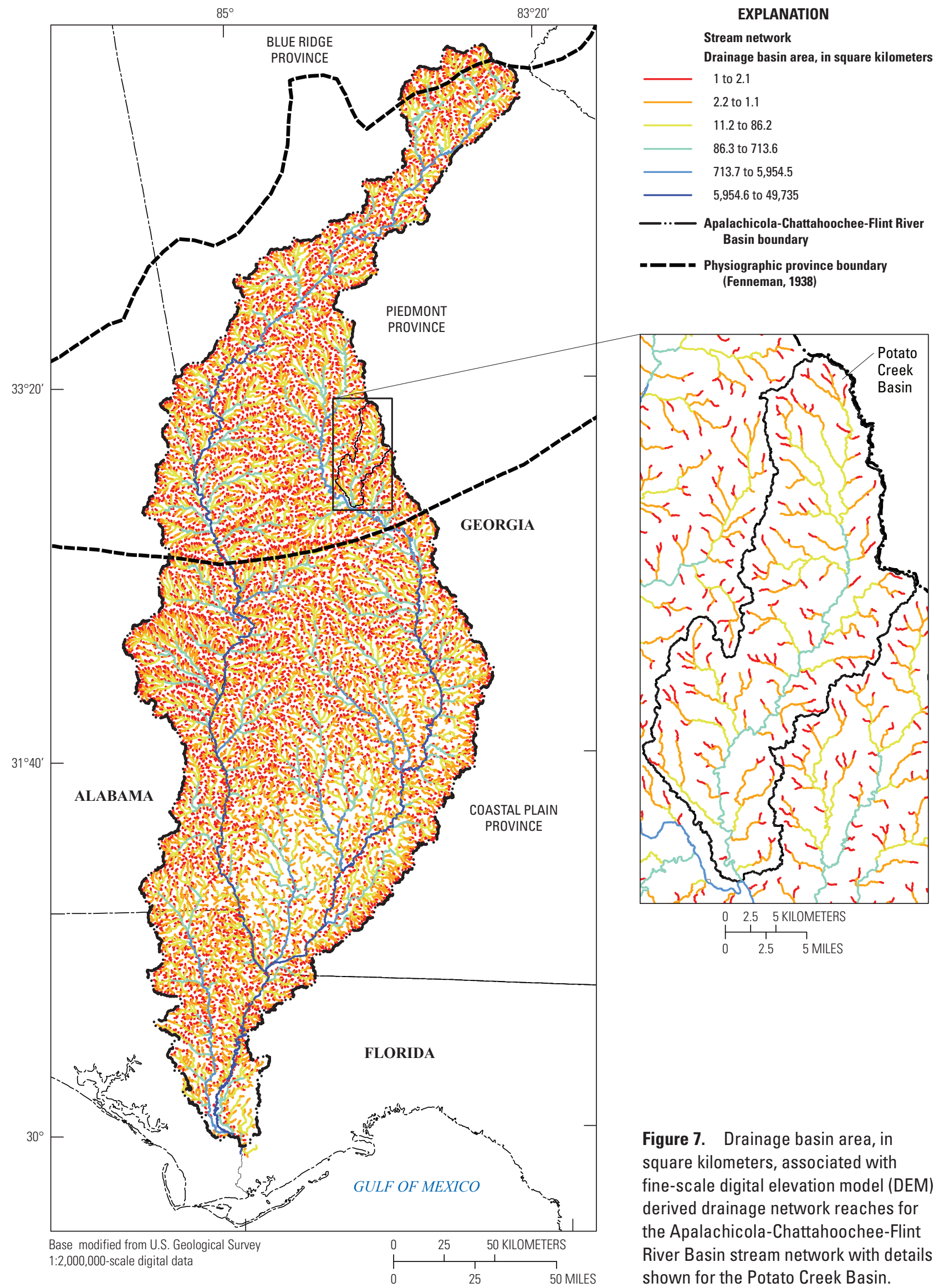

Figure 7. Drainage basin area, in square kilometers, associated with fine-scale digital elevation model (DEM) derived drainage network reaches for the Apalachicola-Chattahoochee-Flint River Basin stream network with details shown for the Potato Creek Basin. 


\section{Scale}

Organization of stream segments into reaches typically attempts to lump sections with homogenous hydrology, geomorphology, and geology at a scale that encompasses multiple channel unit (riffle-pool) sequences (Frissell and others, 1986). In this sense, stream segments break with changes in geology and at junctions of tributaries large enough to cause changes in hydrology. Reaches are within the larger stream segments. In our Apalachicola-Chattahoochee-Flint stream network, segments break at every tributary junction and reaches break within segments with threshold changes in stream-channel slope or valley width (fig. 2). This segmentation was desirable to satisfy fine-scale metapopulation model needs yet maintain higher-resolution reach-scale data.

The segment- and reach-based stream networks developed for this study are composed of identical line representations; however, the fine-resolution stream network is made up of reaches within segments. Reaches are attributed with drainage basin area, channel width, valley floor width, and slope and the geomorphic classification is for the reach scale (fig. 2, appendix table 1-1). Reach-scale data are aggregated to the segment scale with breaks occurring only at tributary junctions in the seven fine-scale model basins used for fish-occupancy modeling (fig. 2, appendix table 2-1). The field GRID_CODE provides the link between the PRMS models and fine-scale occupancy models in the seven fine-scale basins (appendix table 2-1). Segments retain the minimum, maximum, and mean values of each attribute from the reach scale (fig. 2). Each segment has a unique identifier (GridCode) that can link the segment to watershed hydrology, stream temperature, and biologic models. Each segment in the segment-based network also was attributed with an analysis of barriers, stream isolation, and modeled percent urban area from 2000-2100 for the upstream adjacent watershed. The coarse-scale PRMS watershed model and coarse-scale species-response models use a pruned version of the full network and can be viewed using the attribute COARSE=1 (fig. 2, appendix table 1-1).

\section{Channel Width}

Channel width was calculated from regional regression curves relating stream-channel parameters to drainage area. Regional regression curves are available from literature for the southeastern United States (Harman and others, 1999; Doll and others, 2002; Mohamoud and Parmar, 2006; Glickauf and others, 2007; Johnson and Fecko, 2008). Channel width regression curves are of the following form:

$$
w=a A_{d}^{b}
$$

where
$w$ is bankfull width in meters,
$A_{\mathrm{d}} \quad$ is drainage area in square kilometers, and
$a$ and $b \quad$ are empirically derived exponents.

Coefficient values developed for the Piedmont were applied to derive channel widths for stream segments in the Apalachicola-Chattahoochee-Flint ( $a=2.94$ and $b=0.39$ ), an assumption that simplifies some physiographic variability in channel width (Harman and others, 1999; Johnson and Fecko, 2008). Although Johnson and Fecko (2008) recommend province-specific coefficients for the Blue Ridge, Piedmont, and Coastal Plain, one value for the ApalachicolaChattahoochee-Flint River Basin was desired to prevent large changes in width at province boundaries and was considered appropriate for this network-wide approach.

Field measurements, a lidar dataset for the Potato Creek Basin, and a regional streamgage analysis were used to evaluate regional channel geometry derived channel widths based on drainage area. To evaluate channel dimensions using a lidar dataset in the Potato Creek Basin, we randomly selected 85 reaches from the DEM-derived network in the Potato Creek Basin. In each reach, a cross section transect representative of the channel in the reach was drawn perpendicular to the channel and valley, and intersected with the 1-m DEM generated from bare earth lidar data (fig. 8). Estimates of bankfull width and depth, and valley width were measured from the cross sections of the lidar data.

To further assess the accuracy of the regression-based channel width estimates, we analyzed regional channel geometry for 30 USGS streamflow gage locations throughout the Apalachicola-Chattahoochee-Flint River Basin. Building on a dataset created for the Piedmont province (Riley and Jacobson, 2009), gage measurement records from the Blue Ridge and Coastal Plain were analyzed to determine bankfull channel width and depth from field discharge measurements at approximately the 2-year recurrence discharge calculated from peak flow files.

The estimates of channel width from field measurements, lidar measurements, and stream-gaging measurements were joined in a GIS with the drainage-area-based widths generated from regional regression relations. The drainage-area-based predictions of channel widths agree reasonably well with other estimates of bankfull channel width (fig. 9). They generally are consistent with field measurements over the range of drainage areas but underestimate channel measurements made with lidar at drainage areas less than $50 \mathrm{~km}^{2}$ (fig. 9). The drainage-area-derived widths also are somewhat smaller than the channel widths derived from measurements at USGS streamflow-gaging stations (fig. 9). Drainage-area-based streamchannel widths ranged from 1.7-200 $\mathrm{m}$ in the ApalachicolaChattahoochee-Flint River Basin, varying with drainage area and physiography (fig. 10). There is a gap in the distribution of channel widths between 150 and $190 \mathrm{~m}$. This jump occurs where the Chattahoochee and Flint Rivers converge together to form the Apalachicola River. 

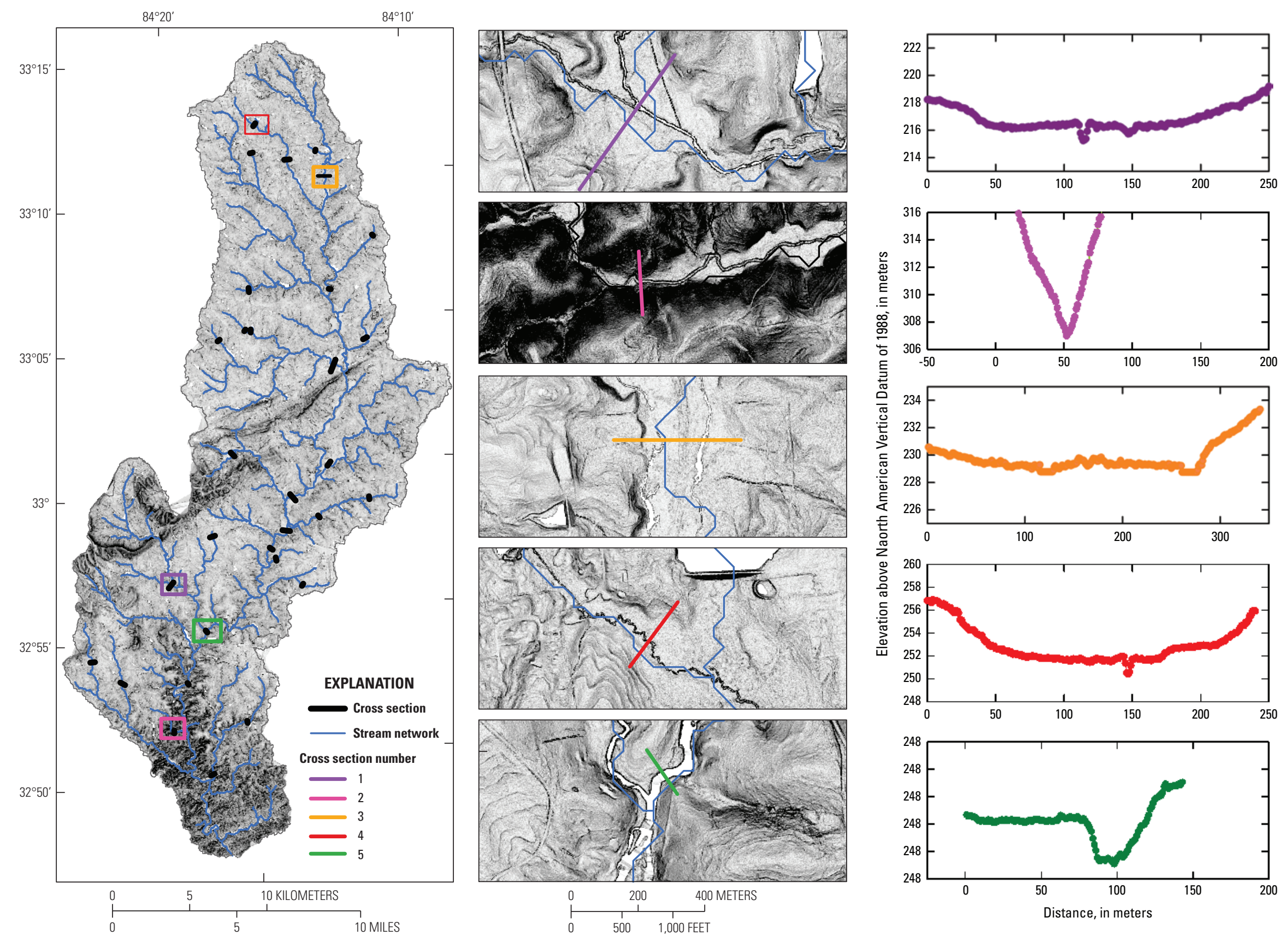

Figure 8. Cross-section geometry derived from the light detection and ranging (lidar) digital elevation model dataset for the Potato Creek Basin. 


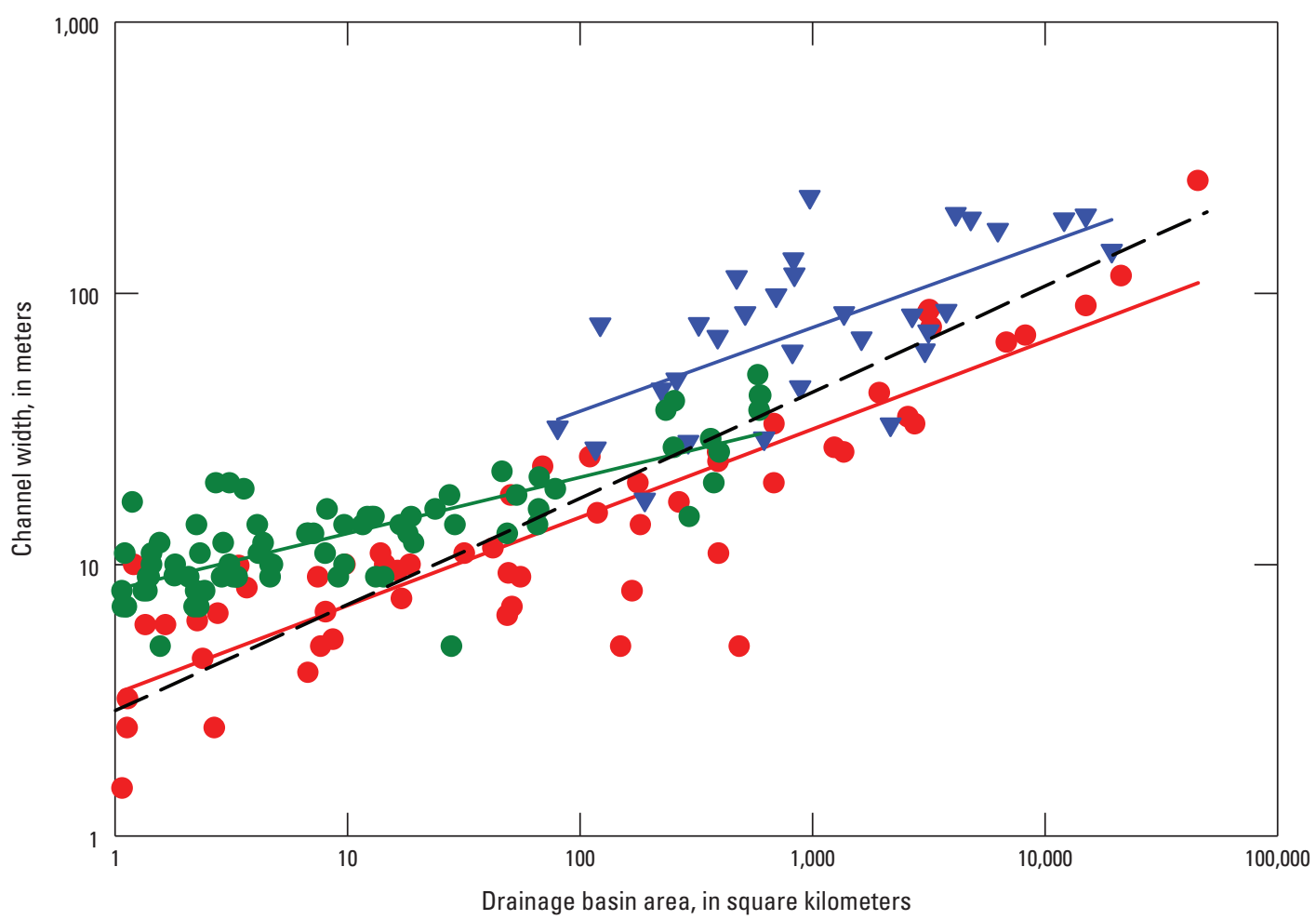

EXPLANATION

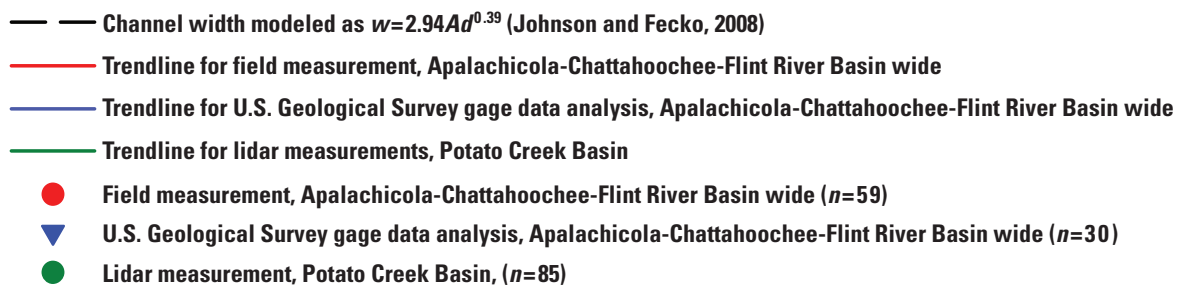

Figure 9. Modeled bankfull channel width comparison from field estimates, U.S. Geological Survey streamflow gaging station data analysis for the Apalachicola-Chattahoochee-Flint River Basin, and light detection and ranging (lidar) digital elevation model measurements for the Potato Creek Basin.

\section{Reach Gradient}

Reach gradient was calculated using a polynomial fit of stream elevations over a 100-m long centered window, which was averaged for each reach (Miller, 2008). Mean reach gradient was 0.0078 and gradient ranged from $0-0.557$ (fig. 11). Stream gradients were higher in the high-relief terrain of the Blue Ridge and where resistant Proterozoic Orthogneiss outcrops in the Piedmont near the fall line (fig. 3). An example of a region of higher stream relief can be seen in the lower reaches of the main-stem channel in the Potato Creek Basin (fig. 11). Low gradient streams are prevalent in the Coastal Plain, large rivers, and reservoirs.

\section{Valley Width}

The valley, or flood-plain, width was calculated using the inundation flow path method in Netrace (Miller, 2008). This method measures valley width by intersecting all cells adjacent to the stream segment in the DEM within a specified elevation of each channel cell. The height above the channel can be set to a multiple of bankfull channel depths, which were derived from regional relations. For calculating valley width, the regional bankfull depth relation was modified:

$$
d x=a A_{d}^{b}
$$




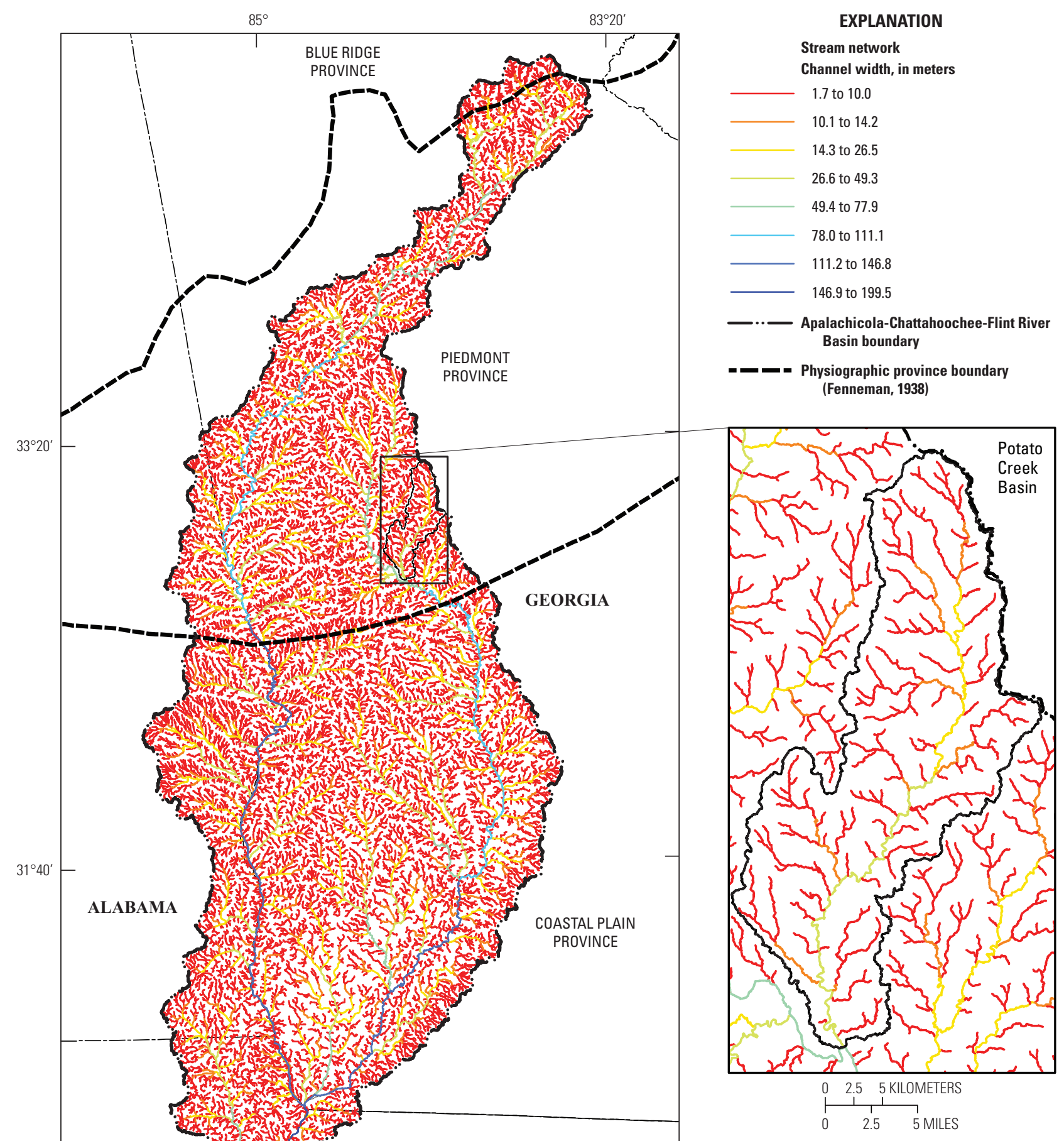

Figure 10. Modeled channel width, in meters, for the ApalachicolaChattahoochee-Flint River Basin stream network with details shown for the Potato Creek Basin. 


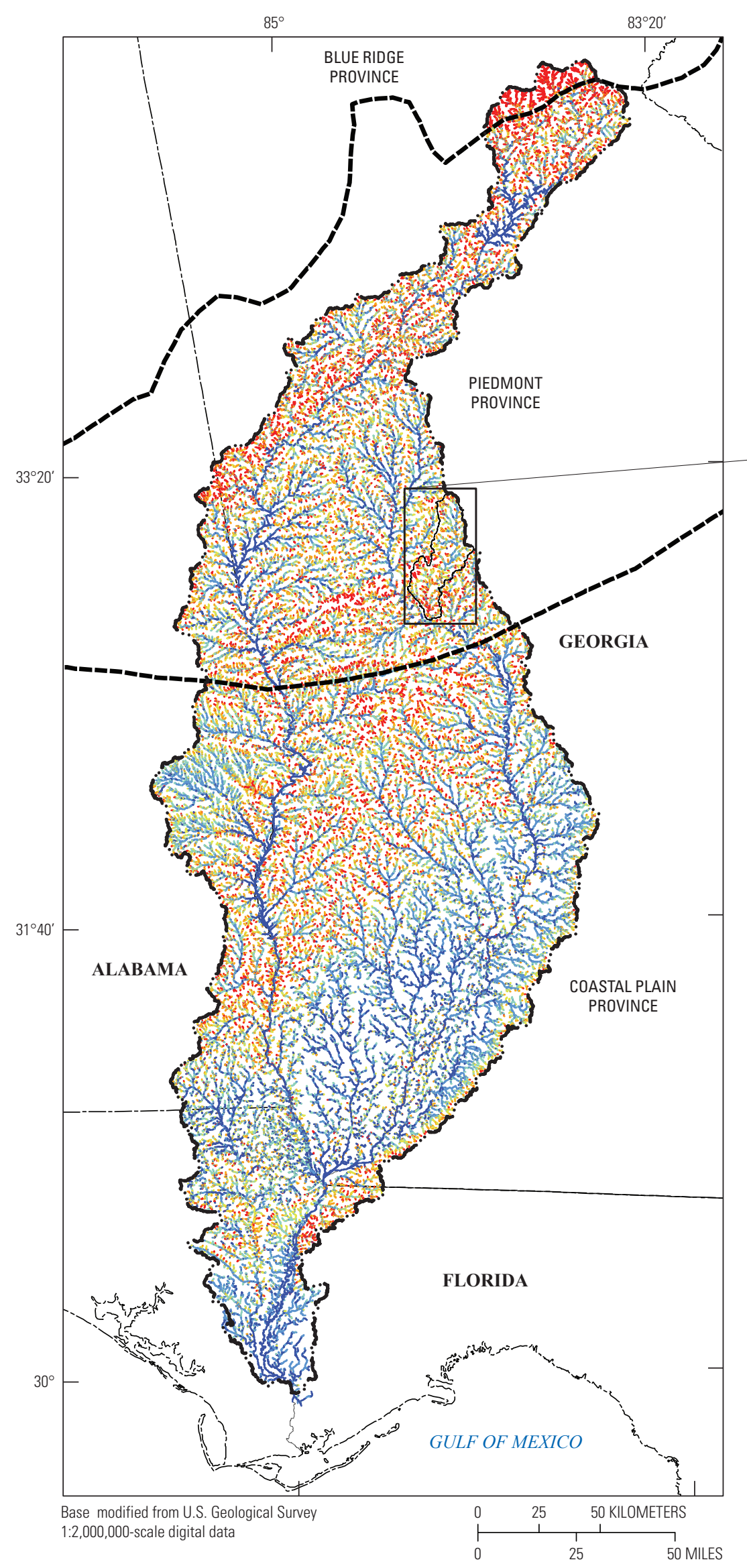

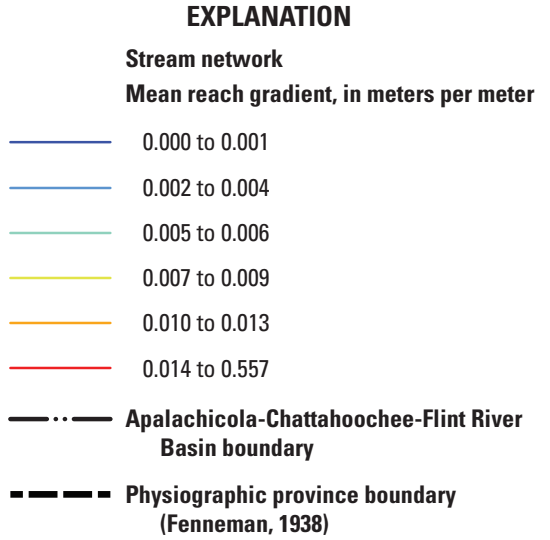

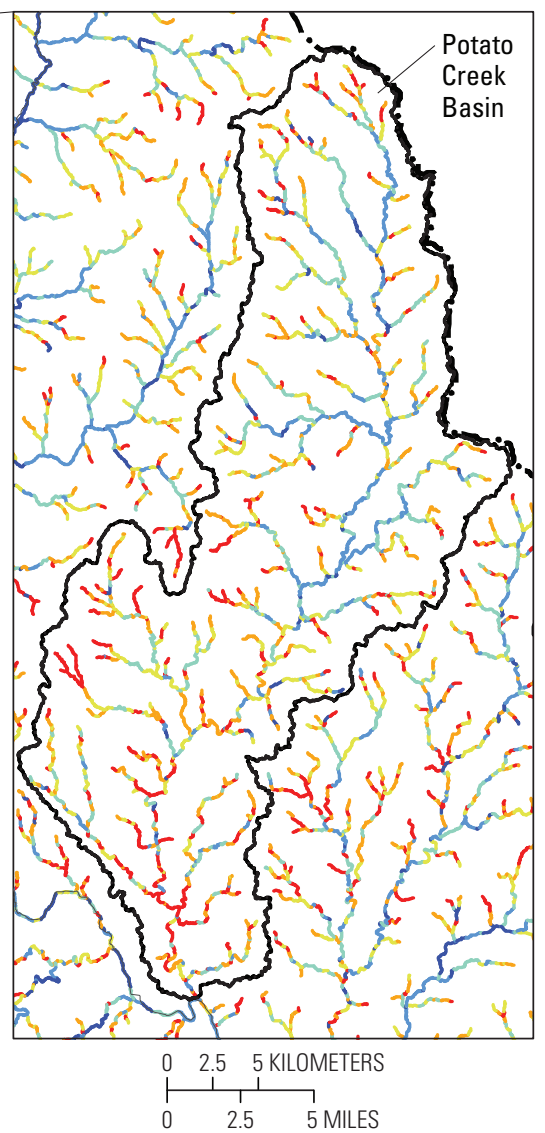

Figure 11. Mean reach gradient for the Apalachicola-Chattahoochee-Flint River Basin stream network with details shown for the Potato Creek Basin. 
where

$$
\begin{aligned}
d \text { is } & \text { bankfull depth in meters, } \\
x & \text { is a multiplier for channel depth, } \\
A_{d} & \text { is drainage area in square kilometers, and } \\
a \text { and } b & \text { are empirically derived coefficients. }
\end{aligned}
$$

Parameter values were selected to be consistent with values determined for the Piedmont ( $a=0.46$ and $b=0.32$ ) (Harman and others, 1999).

Multiplier values for $x$ from 2-3 times channel depth were used to map valley width and results were then compared to lidar-derived valley widths (see example of channel width, fig. 8). The multiplier used to convert bankfull channel depth into the elevation offset was set based on the judgment of the authors. Although resultant valley widths were insensitive to this multiplier for segments with small drainage areas, the multiplier's impact increased with increasing drainage area (fig. 12). A value of two times the bankfull depth produced valley width estimates that closely fit the lidar-derived valley widths in the Potato Creek Basin, although our predicted valley widths generally over predict. Valley width was measured at nine field sites in the Apalachicola-Chattahoochee-Flint River Basin, and the predicted valley width also generally fit field measurements using an elevation criterion of two times the bankfull channel depth (fig. 12). The resulting valley widths calculated for the Apalachicola-Chattahoochee-Flint River Basin ranged from 3-9,740 m (fig. 13). Narrow valleys generally are found in headwater streams throughout the Apalachicola-Chattahoochee-Flint River Basin, particularly in the high-gradient terrain of the Blue Ridge and Piedmont provinces. Wide valleys are prevalent in the Coastal Plain, larger rivers, and in reaches with reservoirs.

\section{Stream Order}

Several measures of stream order were calculated for each segment in the Apalachicola-Chattahoochee-Flint River network using RivEX, a stream-network analysis extension for ArcGIS 9.3 (Hornby, 2010). Strahler stream order designates headwater streams as first order and an increase in stream order of +1 occurs at each entry of a stream the same magnitude (Strahler, 1952). Strahler stream order ranged from
Figure 12. Modeled valley width comparison with field measurements throughout the Apalachicola-ChattahoocheeFlint River Basin, digital-elevation model, and light detection and ranging (lidar) digital elevation model measurements in the Potato Creek Basin. Valley width was modeled using 2, 2.5, and 3 times the lidar-derived bankfull channel depths and 30-meter digital elevation model data.

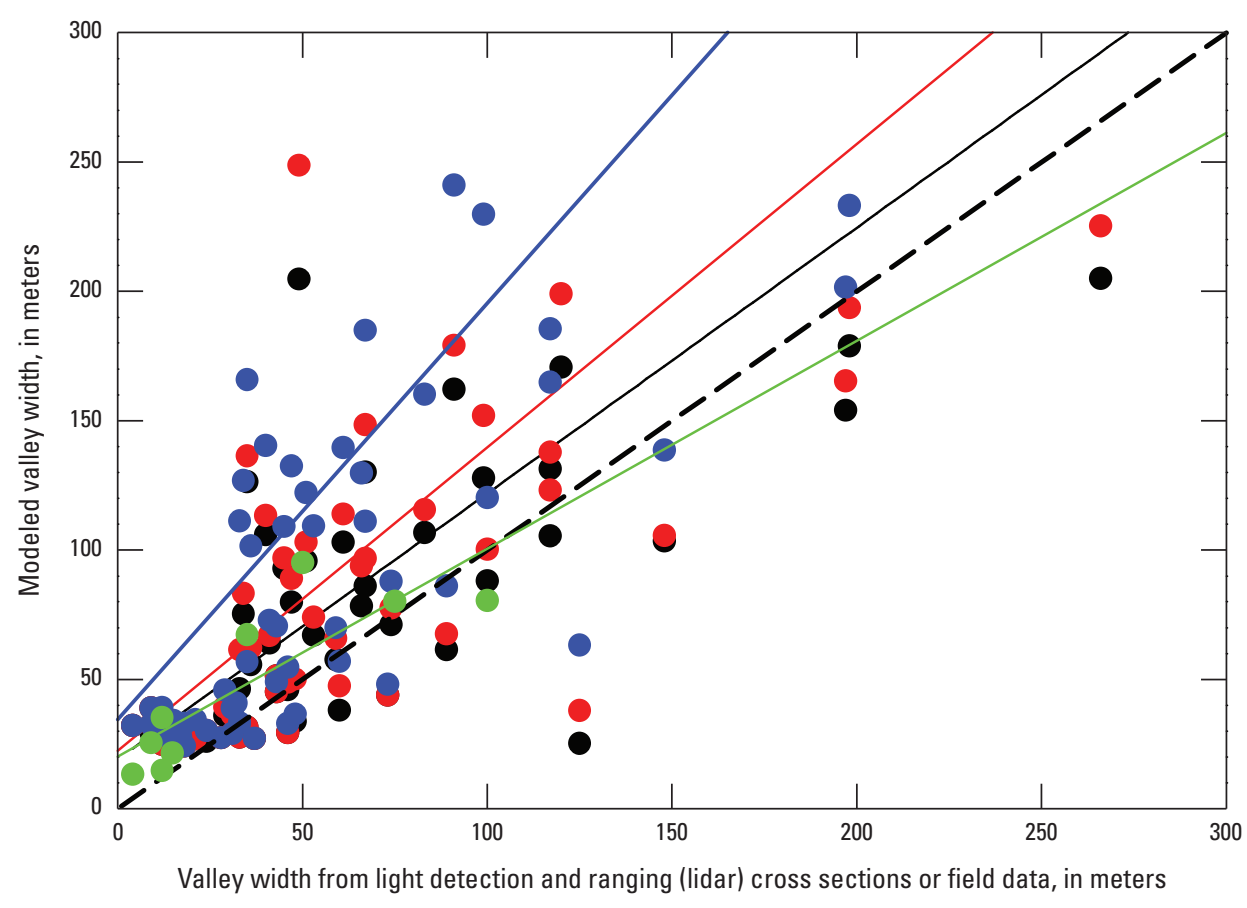

\section{EXPLANATION}

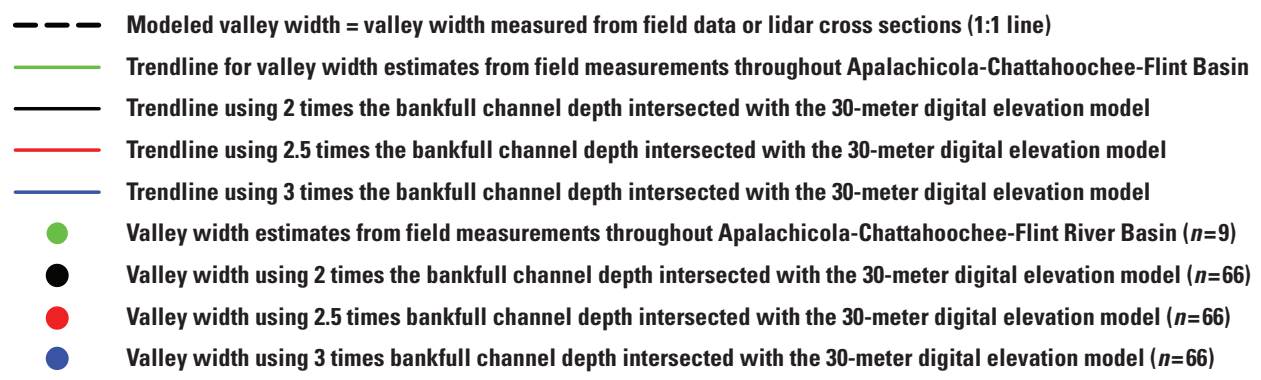




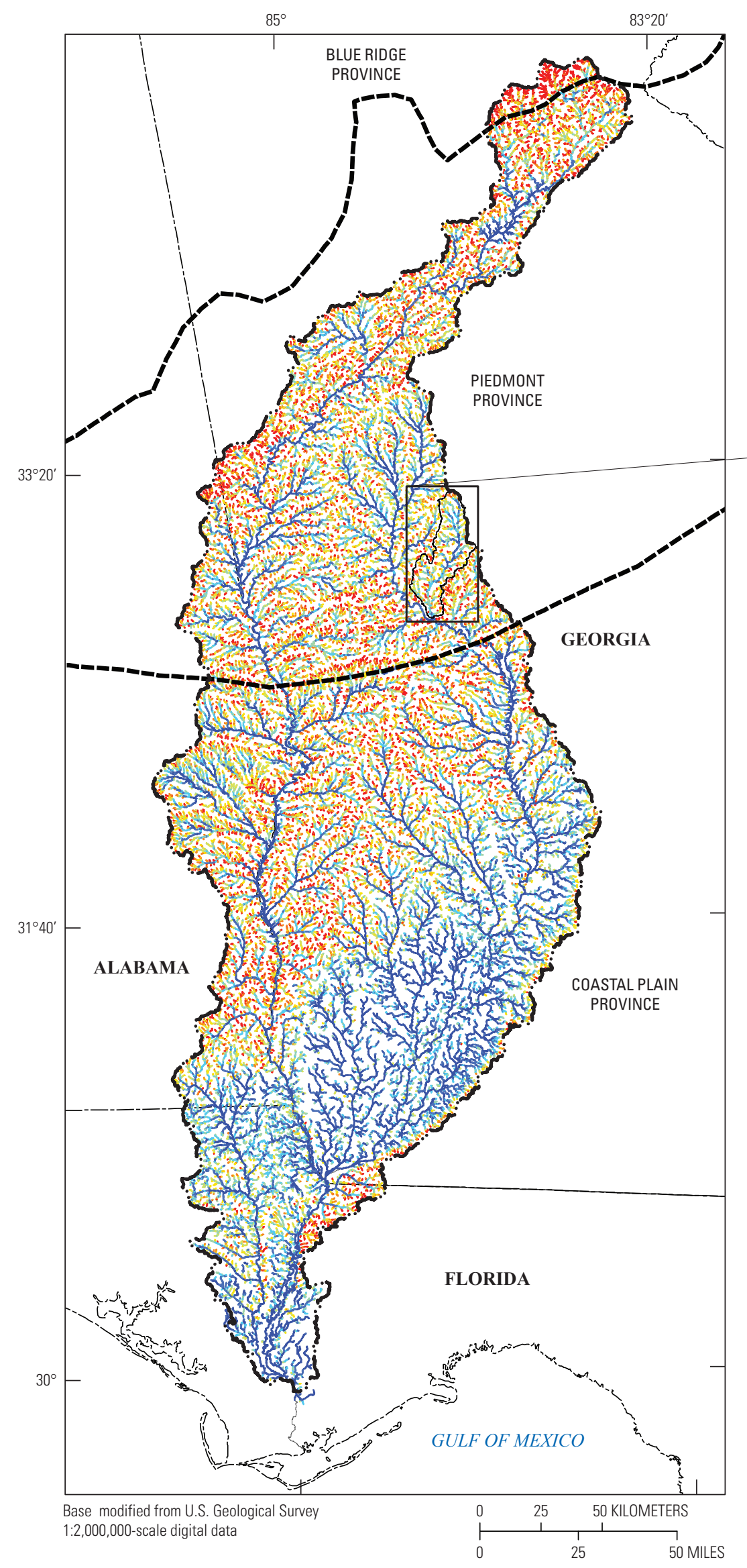

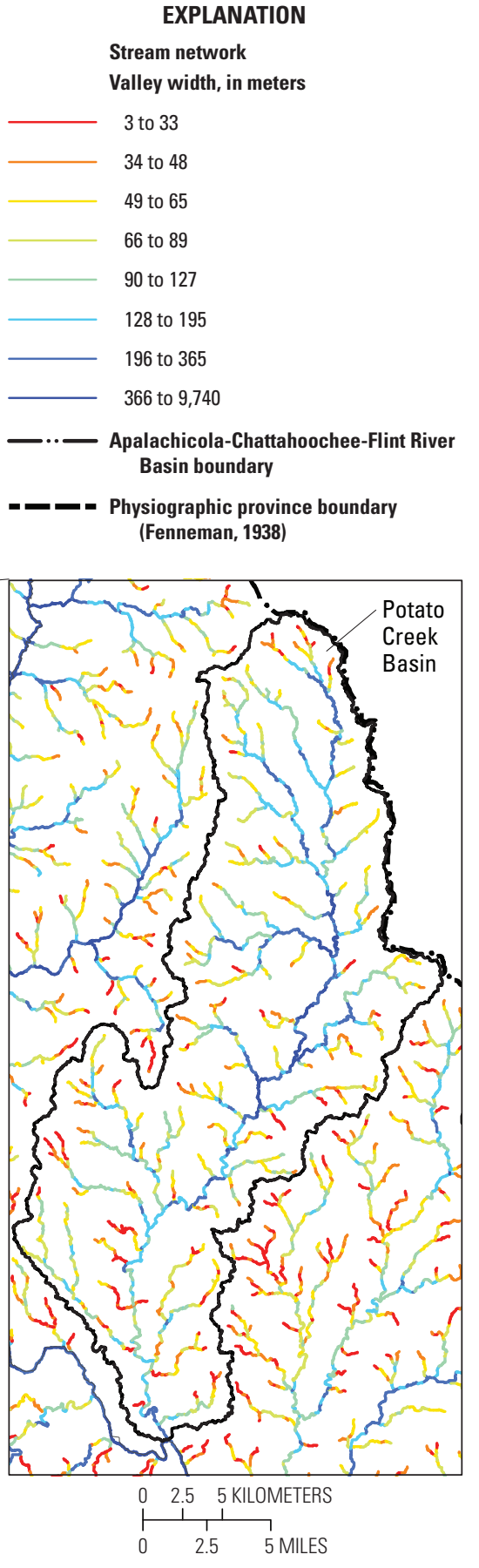

Figure 13. Modeled valley width, in meters, for the ApalachicolaChattahoochee-Flint River Basin stream network with details shown for the Potato Creek Basin. 
1 to 8 in the Apalachicola-Chattahoochee-Flint River Basin (fig. 14). Shreve stream order, or link magnitude, increases as each drainage enters a stream network and also indicates the cumulative number of first order drainages upstream from a given stream segment (measured tributary junction to tributary junction) (Shreve, 1967). The downstream link magnitude (D-Link), or link magnitude of the next segment downstream, was calculated at the segment scale for input into the finescale fish metapopulation models. To calculate D-Link, tables were joined in ArcGIS associating the "to node" number for a segment with the "from node" for the segment immediately downstream and attributing the segment-level fine-network file with the downstream-segment Strahler and Shreve stream orders.

\section{Stream Power Calculations}

Stream power, or the rate of potential energy dissipation against the bed and banks of a river (Knighton, 1998), is a measure that integrates stream-channel gradient and discharge into a quantitative value of power per length of channel.

Stream power is often used as a measure of a stream's capacity to transport sediment or erode its banks:

$$
\Omega=\rho g Q S
$$

where

$$
\begin{aligned}
& \Omega \quad \text { is stream power (watts/meter), } \\
& \rho \quad \text { is the density of water (1,000 kilograms per } \\
& \text { cubic meter), } \\
& g \quad \text { is the acceleration because of gravity } \\
& \text { (9.8 meters per second squared), } \\
& Q \quad \text { is a characteristic discharge (in cubic meters } \\
& \text { per second), and } \\
& S \quad \text { is the channel slope. }
\end{aligned}
$$

The characteristic discharge typically is a mean annual or bankfull value. For the current (2013) attribution of stream power, we calculated mean annual discharge from a regional relation of the form:

$$
Q=a A_{d}^{b}
$$

where

$$
\begin{aligned}
Q & \text { is mean annual streamflow in } \mathrm{m}^{3} / \mathrm{s}, \\
A_{d} & \text { is drainage area in } \mathrm{km}^{2} \text {, and } \\
a \text { and } b & \text { are empirically derived exponents. }
\end{aligned}
$$

Published exponent values for the Piedmont Province $(\mathrm{a}=0.015$ and $\mathrm{b}=0.99)$ were used to estimate mean annual discharge in the Apalachicola-Chattahoochee-Flint River Basin (Mohamoud and Parmar, 2006).

Unit stream power is stream power normalized by stream-channel width, in watts per square meter, and is defined with the following equation:

$$
\varpi=\frac{\rho g Q S}{W}
$$

where

$$
\begin{aligned}
& \omega \quad \text { is unit stream power (watts/meter), } \\
& \rho \quad \text { is the density of water (1,000 kilograms per } \\
& \text { cubic meter), } \\
& g \quad \text { is the acceleration because of gravity } \\
& \text { (9.8 meters per second squared), } \\
& Q \quad \text { is a characteristic discharge (in cubic meters } \\
& \text { per second), and } \\
& S \quad \text { is the channel slope, and } w \text { is stream-channel } \\
& \text { width, in meters. }
\end{aligned}
$$

Unit stream power values for the ApalachicolaChattahoochee-Flint River Basin vary, with higher stream power in the steep reaches in high-gradient terrain such as the Blue Ridge and in bedrock-controlled regions of the Piedmont, where high slopes compensate for low mean annual discharge (fig. 15). Large rivers, such as the lower Flint, Chattahoochee, and Apalachicola Rivers, also have high stream power because of the greater discharge. Reaches with lower stream power are present in the low-gradient streams of the Coastal Plain, and in reservoir reaches. To demonstrate the potential to integrate watershed modeling results with geomorphic variables, we calculated mean annual discharge from regional relations.

Values derived from fine- or coarse-scale watershed modeling results and climate change scenarios could be used to calculate stream power. Full integration with watershed modeling results was beyond the scope of this report.

\section{Sinuosity}

Reach sinuosity was calculated using reach length and the straight-line distance between reach endpoints:

$$
S=\frac{D}{D_{s}}
$$

where

$$
\begin{aligned}
& D \text { is the length of the reach or segment, and } \\
& D_{s} \text { is the straight-line distance between the } \\
& \text { endpoints. } \\
& D_{s} \text { is calculated with the following equation: } \\
& D_{s}=\sqrt{\left(x_{1}-x_{2}\right)^{2}+\left(y_{1}-y_{2}\right)^{2}}
\end{aligned}
$$

where

$x_{1}, y_{1}$ and $x_{2}, y_{2}$ are reach or segment endpoint coordinates.

Variations in reach length and the cell size of the DEM $(30 \mathrm{~m})$ may result in sinuosity values that will differ from those developed from aerial photography, field, or lidar data.

Sinuosity values calculated for meandering low-order streams, particularly in low-gradient flood plains, are likely to be underestimated using a 30-m DEM-derived network because the network generating algorithm does not capture actual total stream length. Sinuosity measurements developed 


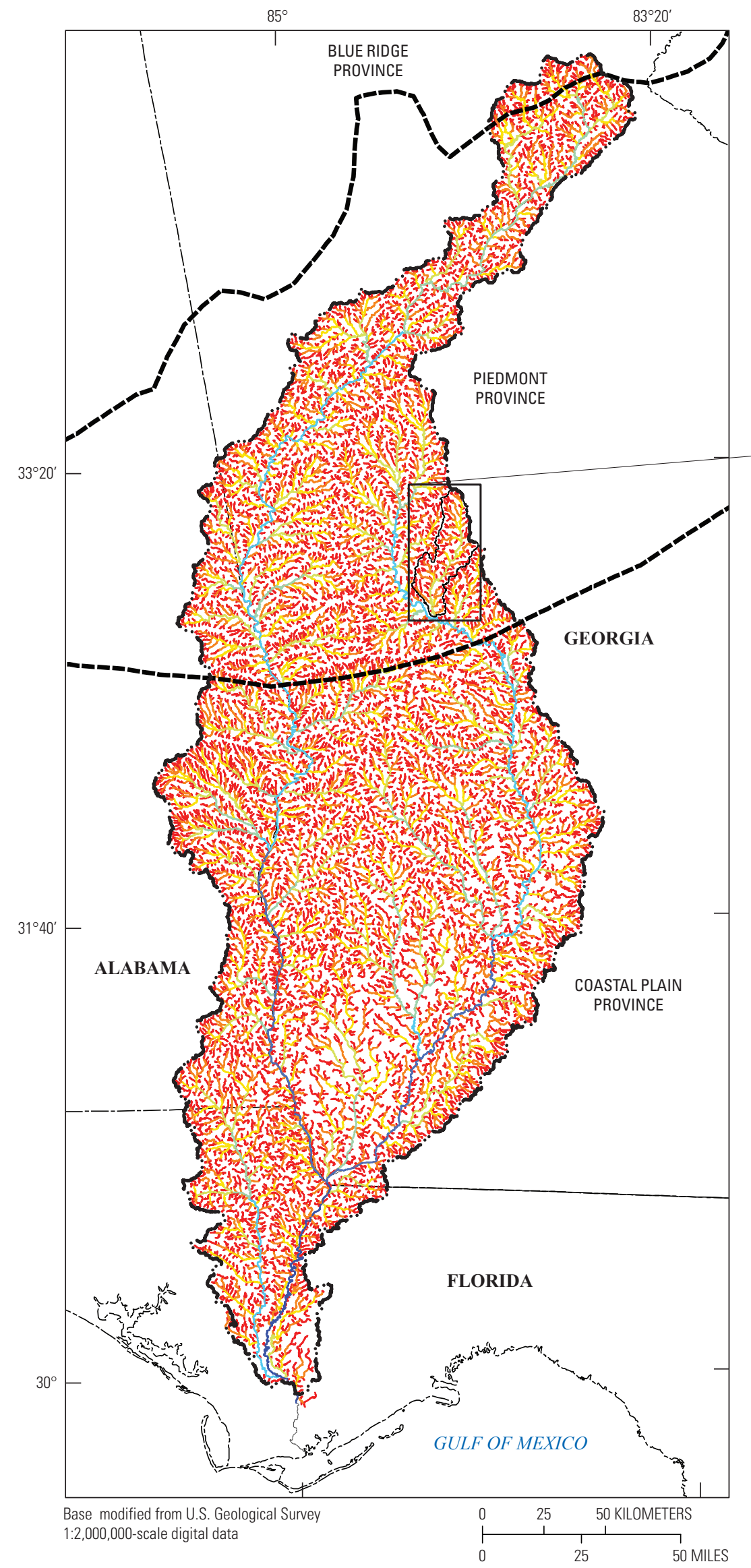

EXPLANATION

Stream network

Strahler stream order (Strahler, 1952)

$-1$

\begin{tabular}{ll}
\hline & 2 \\
& 3 \\
& 4 \\
& 5 \\
& 5 \\
& 6 \\
& 7 \\
\hline & 8
\end{tabular}

-..- Apalachicola-Chattahoochee-Flint River Basin boundary

- - - Physiographic province boundary (Fenneman, 1938)

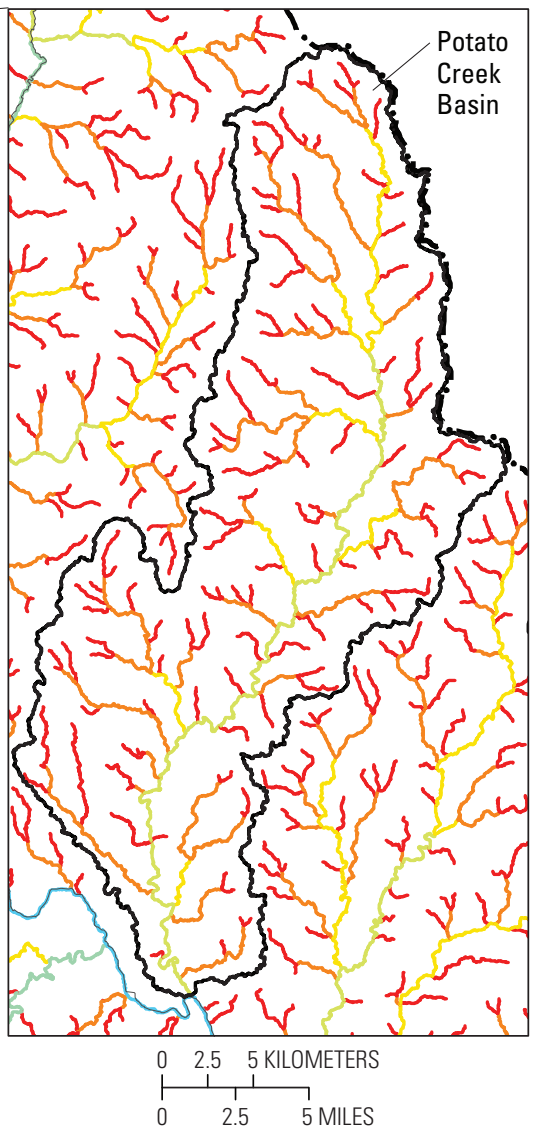

Figure 14. Strahler stream order for the Apalachicola-Chattahoochee-Flint River Basin stream network with details shown for the Potato Creek Basin. 


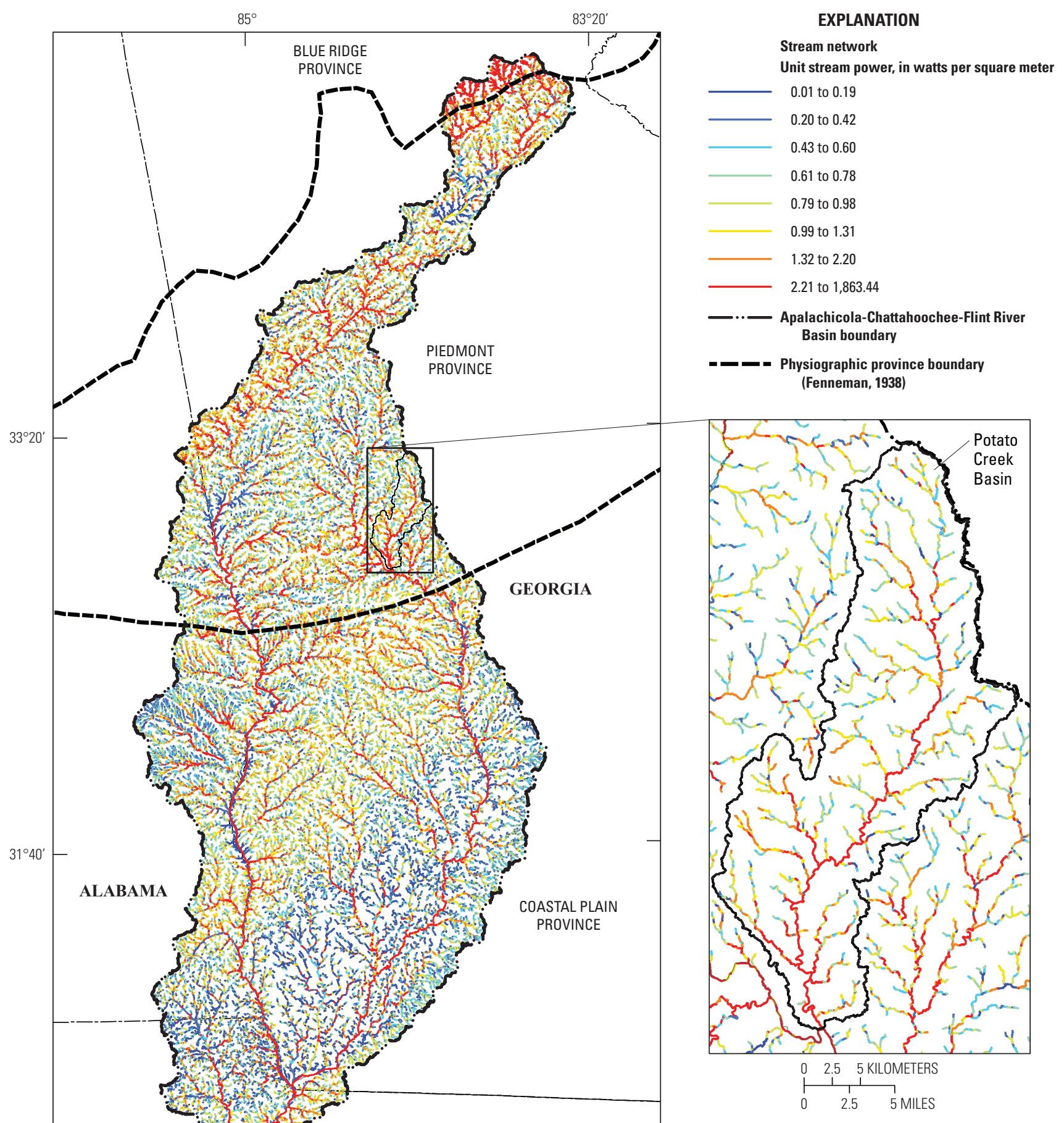

Figure 15. Unit stream power for the Apalachicola-Chattahoochee-Flint River Basin stream network with details shown for the Potato Creek Basin. 
from aerial photography, field, or lidar data would be more accurate than the measurements derived for this study; the DEM-derived stream network was used to calculate sinuosity because it provided a systematic, consistent set of measurements throughout the Apalachicola-Chattahoochee-Flint River Basin. Sinuosity measurements vary with scale as well, and a river with a sinuous network pattern may represent a narrow bedrock stream in a sinuous narrow valley, or a sinuous stream in a river flood plain, such as the Apalachicola River in the Coastal Plain. Sinuosity, when combined with stream confinement ratio, can provide a measure of a stream's ability to move within its flood plain.

In the Apalachicola-Chattahoochee-Flint Basin, stream sinuosity values ranged between 1-4.49 (fig. 16). A threshold of 1.5 was chosen to classify between straight and meandering streams (Leopold and Wolman, 1957). Streams with a sinuosity of greater than two were classified as very sinuous. Straight streams are dominant in the basin; however, there are a number of reaches that have higher sinuosity, particularly in the wider valleys of the Coastal Plain.

\section{Stream Confinement}

We quantified stream confinement as the ratio of floodplain width to channel width. This approach has been used in the Pacific Northwest to differentiate streams able to develop multithread or sinuous channel patterns (Beechie and others, 2006; Reidy Liermann and others, 2011). Beechie and others (2006) hypothesized that narrow streams may not easily erode their banks until they reach a threshold stream-channel width and depth below the typical rooting depth of flood-plain vegetation; that channel migration occurs when channels reach a threshold bankfull width of between $15-20 \mathrm{~m}$ in forested flood plains. In addition, streams with a flood-plain width to channel width ratio of less than four were determined to lack sufficient space to develop a meandering channel pattern with a sinuosity greater than 1.5 (Beechie and others, 2006). "Confined" streams for the Apalachicola-Chattahoochee-Flint River Basin were therefore defined as streams less than $10 \mathrm{~m}$ wide or with a ratio of valley width to modeled channel width less than four.

Confined streams are present throughout the Apalachicola-Chattahoochee-Flint River Basin and generally are small headwater streams or bedrock reaches of larger streams (fig. 17). An example of a bedrock reach of a larger stream can be seen in the downstream reaches of the main stem of Potato Creek (fig. 18), where Proterozoic Orthogneiss outcrops and creates a steep, narrow, bedrock canyon reach.

\section{Barrier and Stream Isolation}

Because dams of various scales, culverts, impoundments, and other structures can fragment stream networks and present barriers to the movement of aquatic species, characterizations of these barriers were derived at the segment scale in the seven fine-model basins. Quantifying the location and degree to which an impoundment or structure impedes fish movement is a challenge and often requires compilation of data from multiple sources. In the case of culverts, small dams, or flood-plain waterbodies near the stream-channel, identification usually requires field investigations.

Multiple data sources were used to determine the location of likely barriers to fish passage for the seven fine-scale model basins. The National Inventory of Dams (NID) (U.S. Army Corps of Engineers, 2010) was consulted to determine the locations of major barriers that are contained within that database. Dams in the NID meet at least one of the following criteria: high or significant hazard classification, equal to or exceeding 25 feet in height and exceeding 15 acre-feet in storage, or equal to or exceeding 40 acre-feet storage and exceeding 6 feet in height. Smaller, private, or more recent impoundments are not contained in the NID. Soil maps (Soil Survey Staff, 2012), the NHD, National Wetlands Inventory (NWI) (U.S. Fish and Wildlife Service, 1994-2000), and an Apalachicola-Chattahoochee-Flint River Basin water-body layer compiled from Landsat 5 Thematic Mapper imagery (Viger and others, 2011; LaFontaine and others, 2013), were all consulted to identify the locations of barriers or waterbodies not included in NID. The NHD and the NWI include polygons mapped as lakes, ponds, or reservoirs. Soil polygons mapped with the attribute "water" in the Soil Survey Geographic database (SSURGO) also were overlain with photography and other impoundment layers to identify the locations of impoundments that intersect the stream. Small run-of the river dams, culverts, and other small features were not assessed for this project.

Barriers were mapped from 1-2-m pixel size National Aerial Inventory Program aerial photography (NAIP) from 2009-10 taken during the growing season. Visual verification and judgment was used to determine if the lake, pond, or reservoir appeared to intersect the stream on the NAIP photography because occasionally sites mapped as water, especially in the Coastal Plain, were natural flood-plain wetlands or offchannel lakes. The Apalachicola-Chattahoochee-Flint River Basin water-body layer also was consulted and was particularly useful in the upstream-most parts of the watershed where the stream itself was too narrow to be classified as water.

Point representations of barriers were intersected with the stream network within a GIS. Stream segment attributes indicate if it contains a barrier. Stream segment attributes also indicate if a segment is upstream from a barrier and is therefore isolated from aquatic species migrations (figs. 19-20). Further attributes indicate if a segment is more than 10 segments upstream from a barrier. This condition occurred only in the Upper Chattahoochee and Chestatee River Basins. In the seven model basins, the percent of stream segments with barriers ranged between 4.7 and 24.0 percent (fig. 21, table 1). The Upper Roanoke Basin has the lowest percentage of stream segments with barriers, and Potato Creek Basin has the highest percentage. 


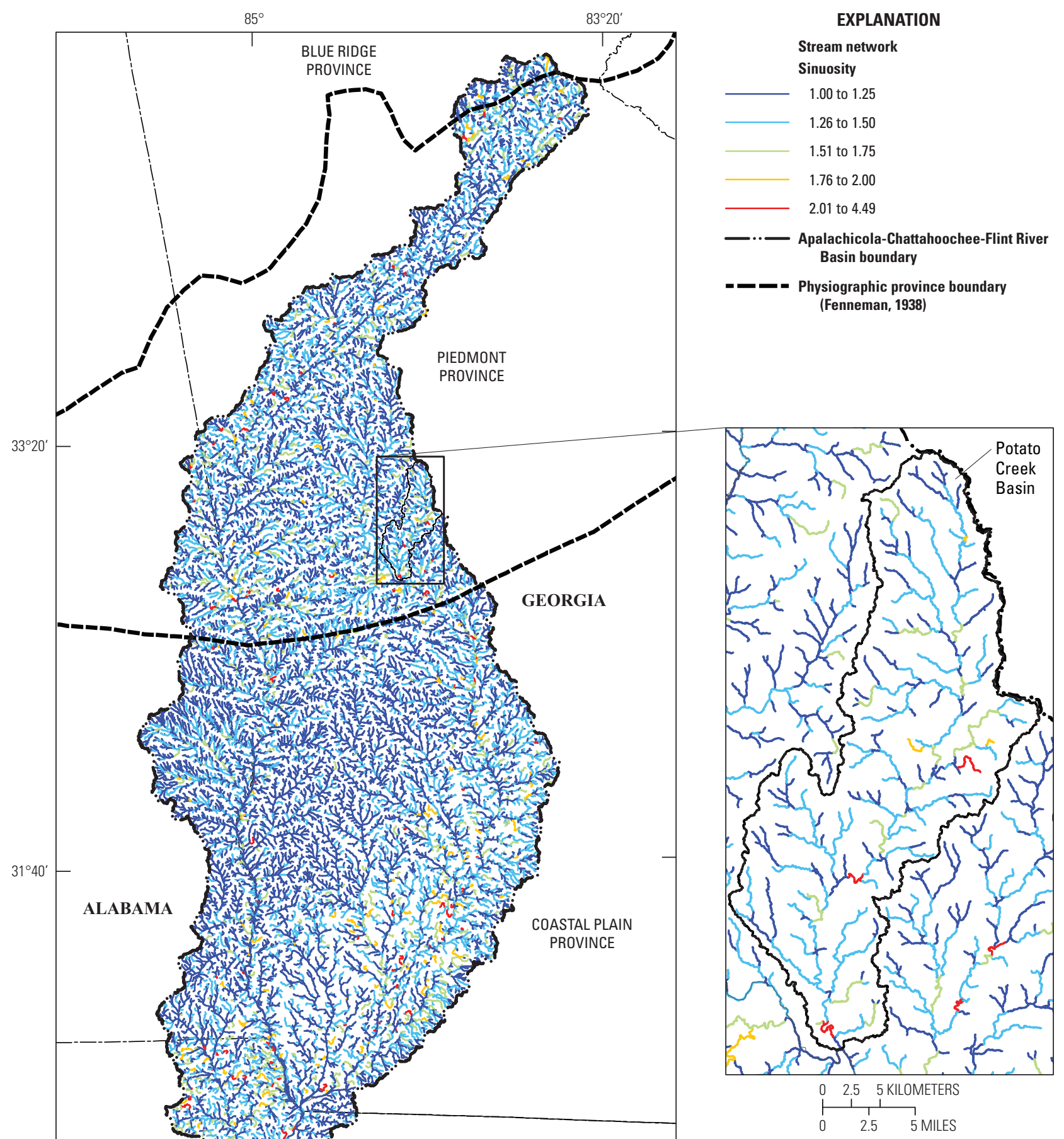

Figure 16. Sinuosity for the Apalachicola-Chattahoochee-Flint River Basin stream network with details shown for the Potato Creek Basin. 

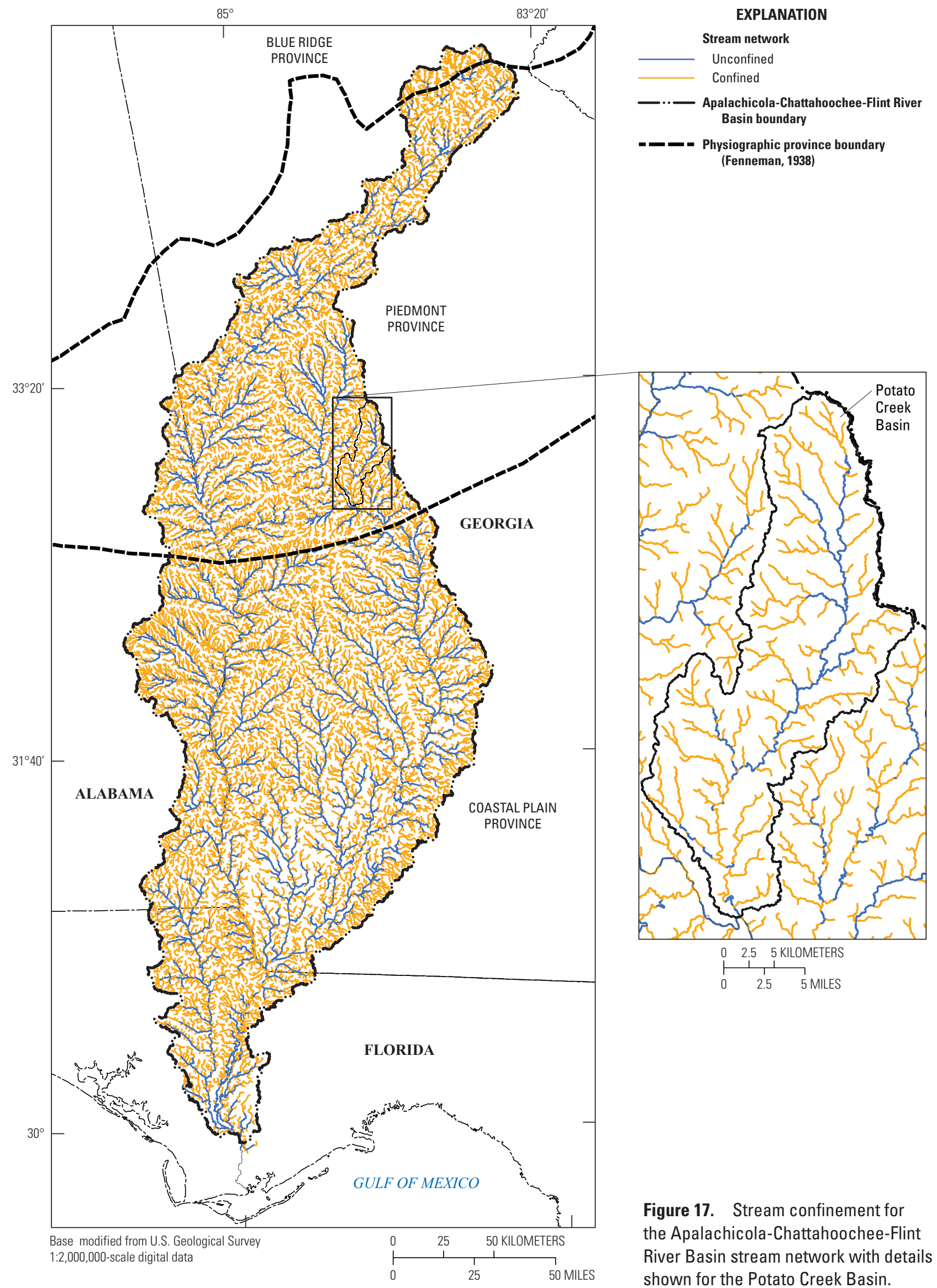

Figure 17. Stream confinement for the Apalachicola-Chattahoochee-Flint River Basin stream network with details shown for the Potato Creek Basin. 
The location of a barrier in a stream network can have a substantial effect on the degree of biotic accessibility of the network segments and on biotic processes. Although the Chestatee River Basin has a relatively low number of barriers (10 percent of segments containing barriers), 70 percent of its segments are isolated because impoundments are located in the downstream part of the basin. This is contrasted by the Potato Creek Basin, which has the highest percent of segments with barriers ( 24 percent), but a lower percent of isolated reaches (45 percent) than the Chestatee River Basin, because barriers are located in the upstream end of the basin. There were fewer isolated stream segments in the Coastal Plain basins (Ichawaynochaway Creek, Spring Creek, and the Chipola River Basins) and the Upper Roanoke River than in the Piedmont and Blue Ridge basins (Upper Chattahoochee River, Chestatee River, and Potato Creek River Basins).

\section{Urbanization}

Urbanization is a likely stressor on stream biotic communities because of potential effects on runoff, water quality, water temperature, sediment yield, and direct stream-channel disturbance. To assess potential for urban effects, each segment in the segment-scale stream network was attributed with the percent urban area in the immediately adjacent land surface, or hydrologic response unit (HRU). Percent urban area was determined from probability models developed by SERAP collaborators with the USGS and North Carolina State University using SLEUTH-3r (Jantz and Goetz, 2005). The SLEUTH model uses slope, hillshade, excluded areas (such as protected areas and parks), urban and transportation inputs, and economic growth cycles to develop probability surfaces for urban growth scenarios. Urbanization was modeled annually at a 60-m resolution for 2000-2100 and aggregated on a decadal basis (Belyea and Terando, 2012) (fig. 22). For application to the stream network and species-response models, pixels with greater than 50 percent probability of urbanization were classified as urban for each decade from 2010 to 2100 . The gridded surfaces were intersected with HRUs corresponding to each stream segment in the seven fine-scale model basins, and percent urban area was calculated for each HRU at each time step (Viger and others, 2010). Segments in the seven coarse basins are populated with attribute information about the percent of associated HRU area with a probability of greater than 50 percent urban for each decade from 2010 to 2100 .

Urban growth trends vary spatially (fig. 23). In 2010, the Upper Roanoke Basin had the highest total percent urban (19 percent), but little growth is predicted from 2020 to 2100; however, the percent urban of the Potato Creek Basin is projected to increase from 11 to 61 percent from 2010 to 2100. Higher probabilities of urban growth are associated with basins in close proximity to a major metropolitan area, such as Potato Creek, which is adjacent to Atlanta, Georgia (fig. 22).
Basins with a high percentage of steep terrain, especially in conjunction with protected areas such as National Forest lands seen in the Upper Roanoke, Upper Chattahoochee, and Chestatee River Basins, have relatively flat growth trends or grow to a point and flatten out as low-lying available land within the basin is populated (figs. 22 and 23).

\section{Coarse-Scale Species-Response Model Inputs}

The coarse-scale species-response model was designed to assess the effects of climate and landscape change on freshwater biota using a spatially explicit expert-opinion based model for the 2000-2100 time period, focusing on stream fishes and mussels (Rieman and others, 2001; Peterson and others, 2010). The coarse-scale species-response model uses the spatial resolution of HRUs and a Bayesian Belief Network associated with the coarse-watershed model and that simulates daily streamflows for the Apalachicola-Chattahoochee-Flint River Basin (LaFontaine and others, 2013). In the watershed model, the Apalachicola-Chattahoochee-Flint Basin was divided into $258 \mathrm{HRUs}$ with a mean area of $200 \mathrm{~km}^{2}$ (fig. 2). The Upper Roanoke Basin is not part of the coarse-scale model. Each HRU in the coarse-scale species-response model is characterized by the range of conditions within an HRU such that model predictions apply to the scale of an HRU, not an individual stream-network segment (Freeman and others, 2011). The coarse-scale species-response model uses inputs of species traits and life history strategy, current population status, colonization potential, and habitat potential to predict future population status based on varying scenarios of land use and climate change. We developed physical-habitat inputs for predicting habitat potential: stream-size distribution, sediment supply, and stream confinement. These inputs were intended to provide a template for future modeling of stream-channel conditions under climate and land-use change scenarios. Stream attributes such as confinement and size distributions from the fine-scale models were summarized at the HRU level for the coarse-scale species-response modeling process. A model of soil erosion in the Apalachicola-Chattahoochee-Flint River Basin was built to address sediment supply in the coarse-scale species-response model.

\section{Soil Erosion}

Soil erosion was modeled using the Revised Universal Soil Loss Equation (RUSLE) in a GIS (Wischmeier and Smith, 1978). The RUSLE is a widely applied model that is modified from the Universal Soil Loss Equation (USLE) (Wischmeier and Smith, 1978) and uses the following equation to predict soil erosion:

$$
A=R^{*} K^{*} L S^{*} C^{*} P
$$




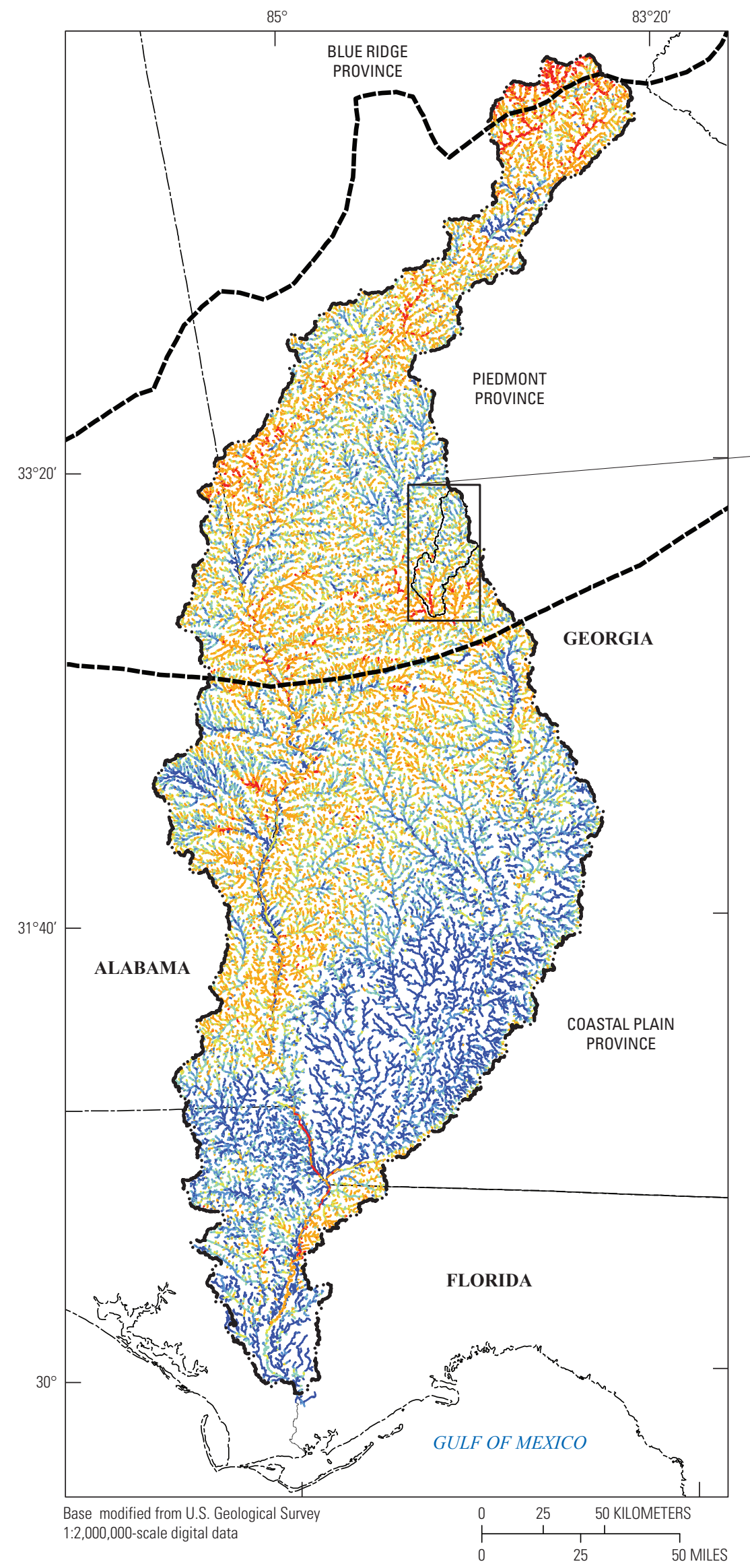

EXPLANATION

Stream network

Ratio of valley width to channel width

1 to 4

5 to 11

12 to 15

16 to 21

22 to 33

34 to 1,015

-..- Apalachicola-Chattahoochee-Flint River Basin boundary

- - - Physiographic province boundary (Fenneman, 1938)

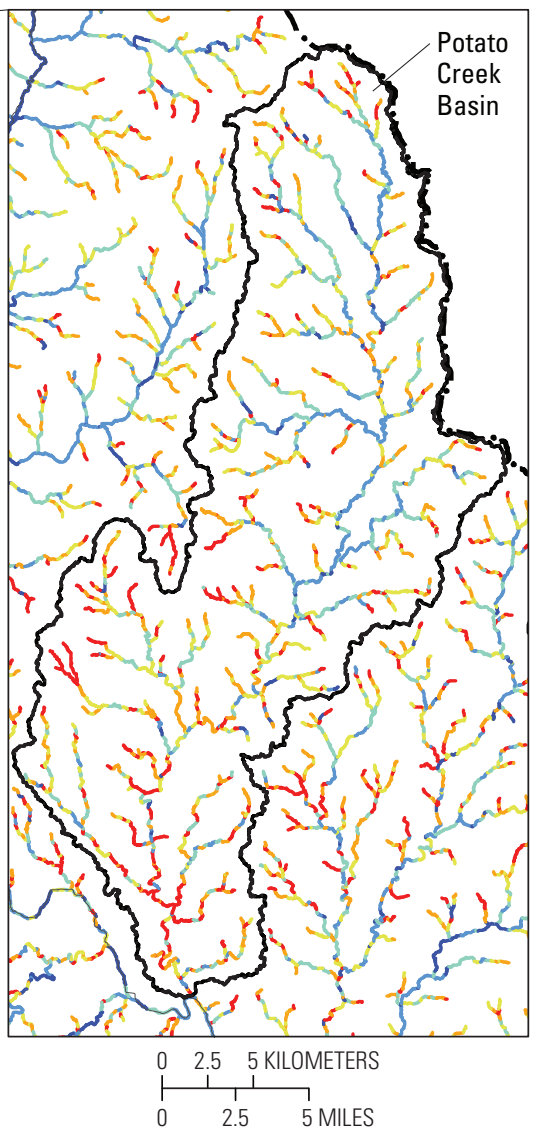

Figure 18. Ratio of valley width to channel width for the ApalachicolaChattahoochee-Flint River Basin stream network with details shown for the Potato Creek Basin. 

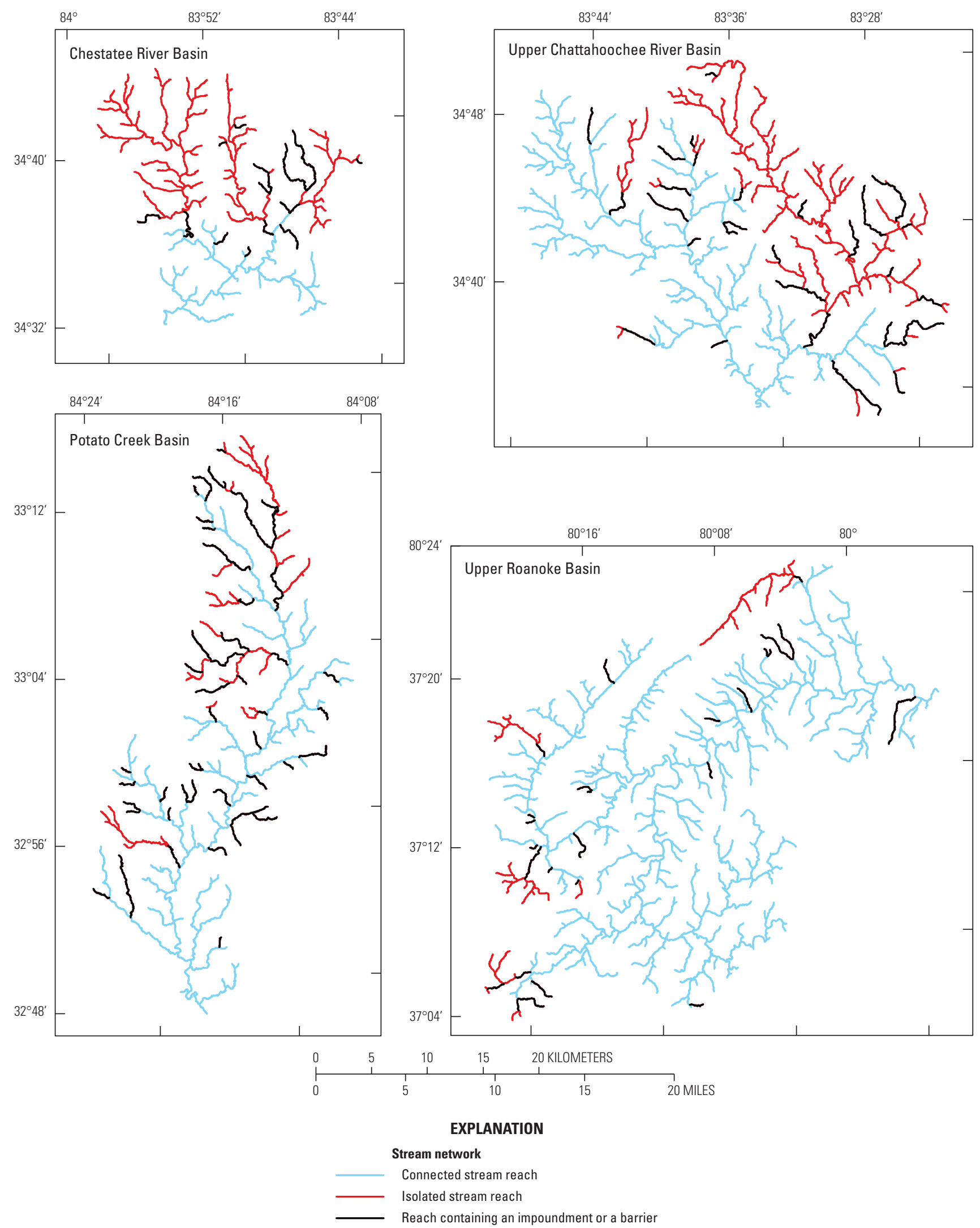

Figure 19. Stream-segment barriers and isolation for the Upper Chattahoochee River, Chestatee River, Potato Creek, and Upper Roanoke River Basins. 

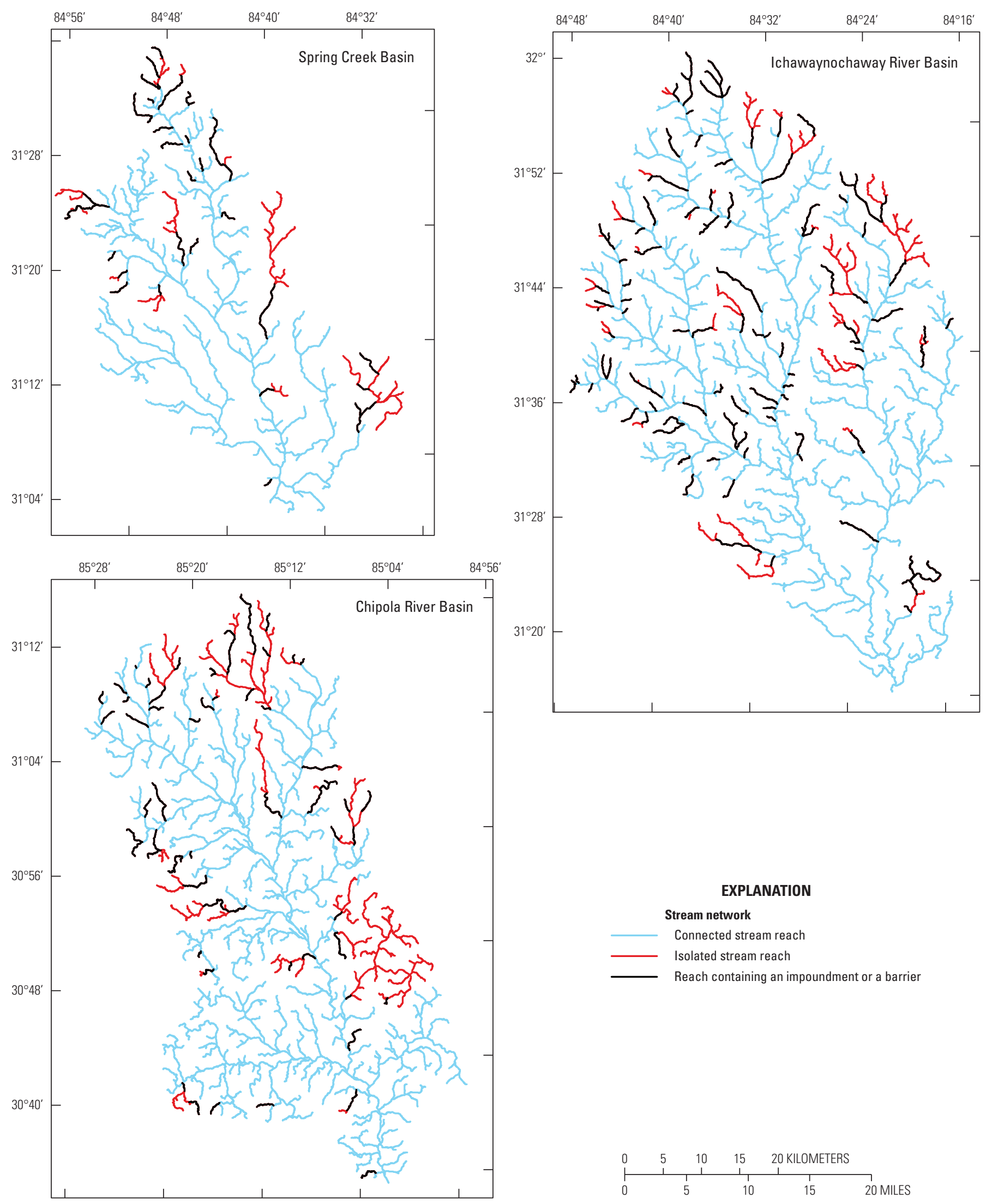

EXPLANATION

Stream network

Connected stream reach

Isolated stream reach

Reach containing an impoundment or a barrier

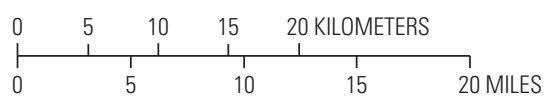

Figure 20. Stream-segment barriers and isolation for the Spring Creek, Ichawaynochaway Creek, and Chipola River Basins. 
Figure 21. Comparisons in the percent of stream segments that contain barriers or are isolated for the seven fine-scale model basins.
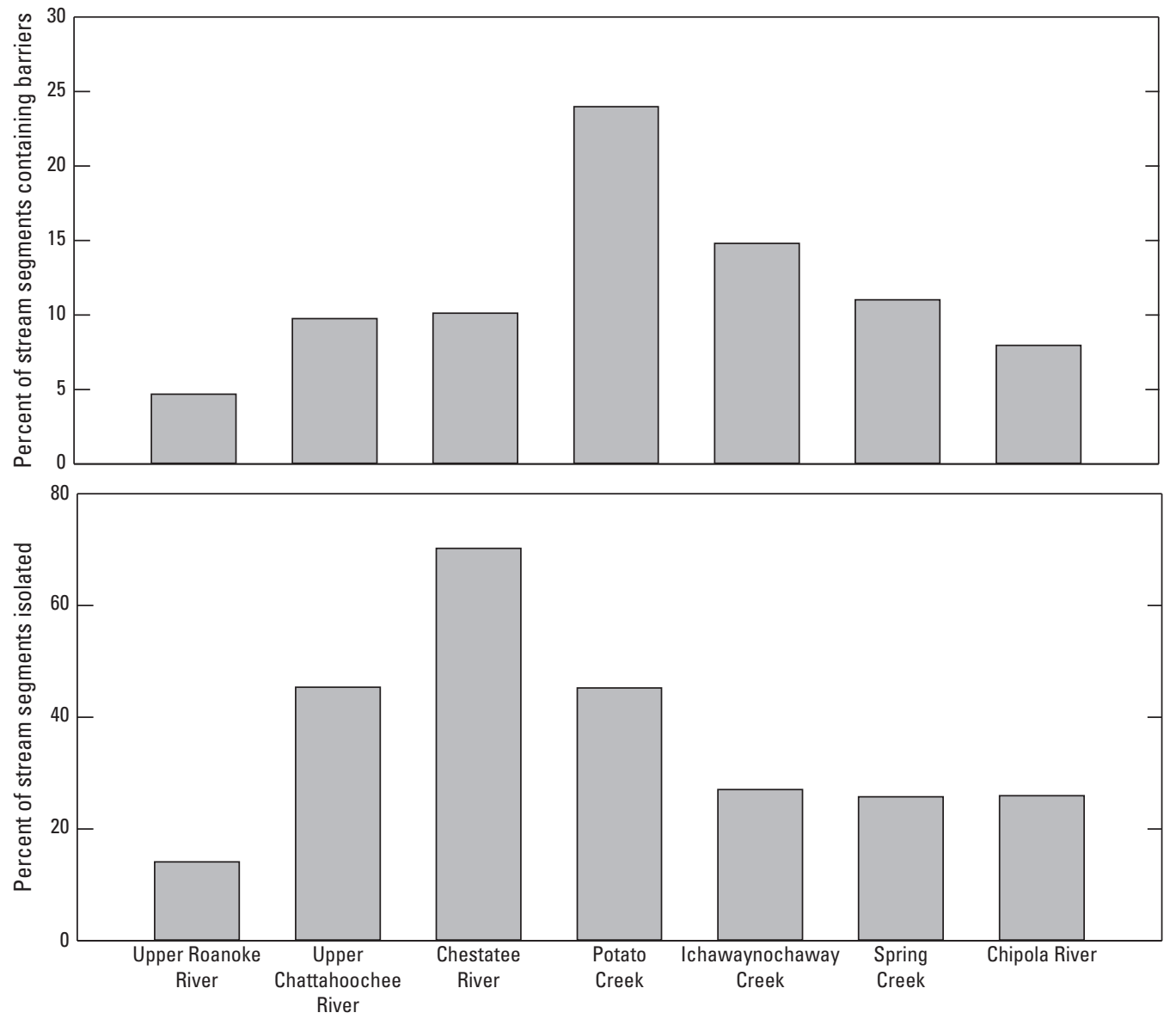

where

$\begin{aligned} A & \text { is the average annual soil loss, in tons per acre } \\ & \text { per year; } \\ R & \text { is the rainfall/runoff intensity; } \\ K & \text { is the soil erodibility; } \\ L S & \text { is the hillslope length and steepness; } \\ C & \text { is the cover and management; and } \\ P & \text { is the support practice. }\end{aligned}$

Soil loss was calculated on a $30-\mathrm{m}$ cell basis and the average value of soil loss in tons per acre per year for a HRU was calculated. The $P$, or support practice factor, was held constant in this application of the RUSLE. This implementation of RUSLE assesses soil erosion at a hillslope scale and does not account for routing of sediment downstream, transport distance, deposition, or stream-channel erosion.

\section{Rainfall Intensity}

The rainfall-runoff erosivity factor represents the effect of rainfall on erosion at a particular location. It is calculated from the total storm kinetic energy times the maximum 30-minute storm intensity. $R$ values were obtained at the county level based on 22-year average annual values and were obtained from a data table for Georgia published by the Georgia Soil and Water Conservation Commission (Georgia Soil and Water Conservation Commission, 2000). The county $R$ values were gridded and resampled to a 30-m grid for the ApalachicolaChattahoochee-Flint River Basin (fig. 24).

\section{Soil Erodibility}

The soil erodibility, or $K$ factor, represents the susceptibility of the soil to erosion and the runoff rate. The $\mathrm{K}$ factor values were obtained from the STATSGO2 soil database (Soil Survey Staff, 2006). In the STATSGO2 database, urban areas have no data, which caused the RUSLE equation to have zero values. For the purposes of calculating soil loss, the soil erodibility for urban areas was populated with the $K$ values for adjacent areas. The modified polygons of STATSGO2 $K$ values were converted into a grid for the entire ApalachicolaChattahoochee-Flint River Basin with 30-m cells and summarized per HRU (fig. 24).

\section{Length Slope Factor}

The terrain or length-slope $(L S)$ factor represents the effect of topography through hillslope length and slope 


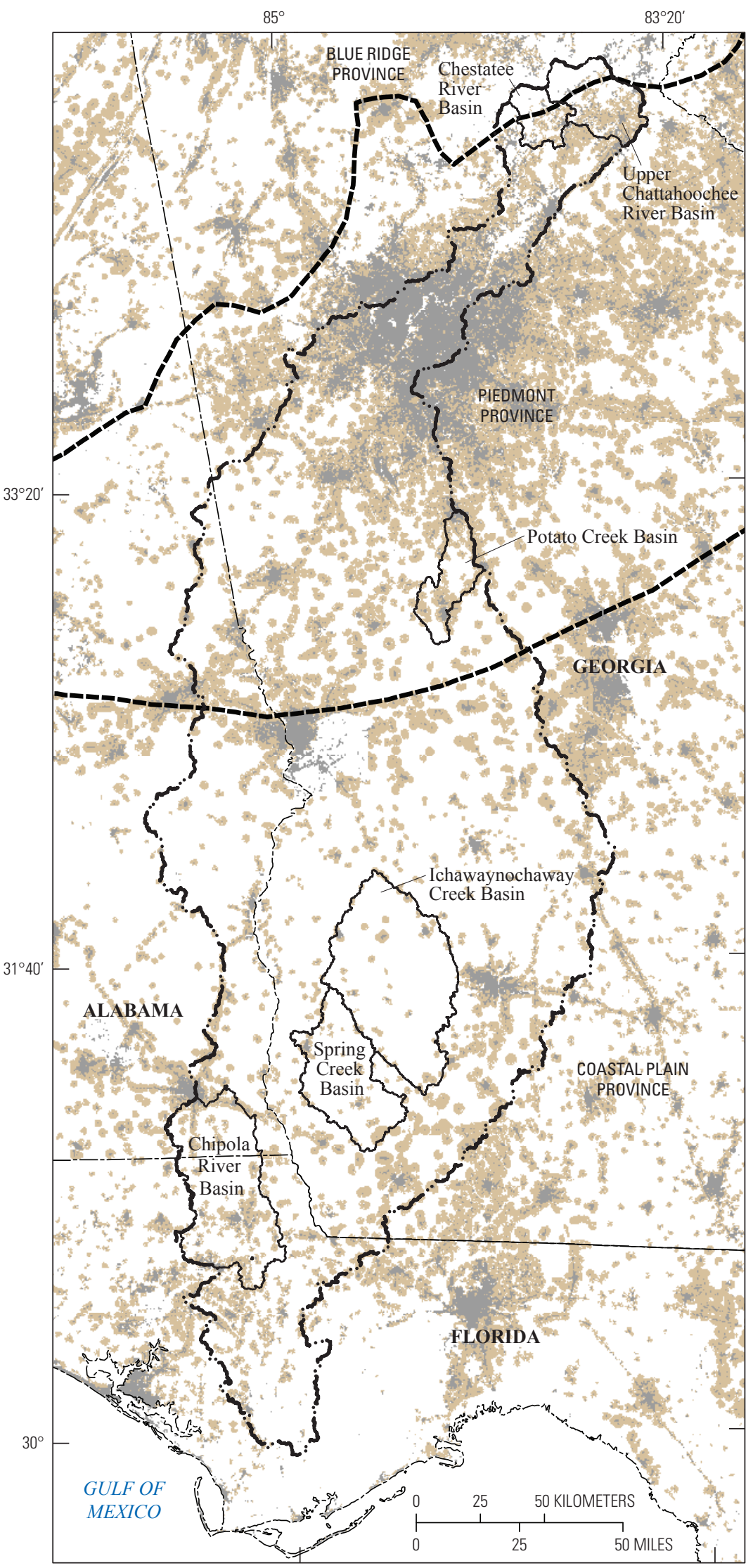

Base modified from U.S. Geological Survey digital data, 1:2,000,000
EXPLANATION
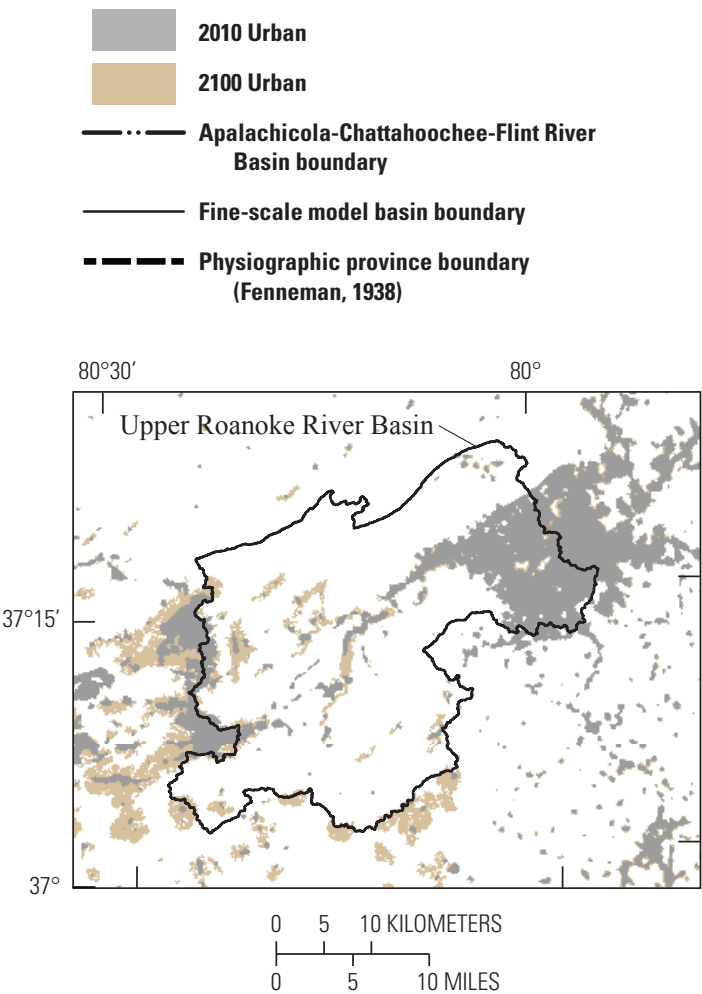

Figure 22. The Apalachicola-ChattahoocheeFlint River Basin with urban land classified with a probability of greater than 50 percent in 2010 and 2100 (Belyea and Terando, 2012). 


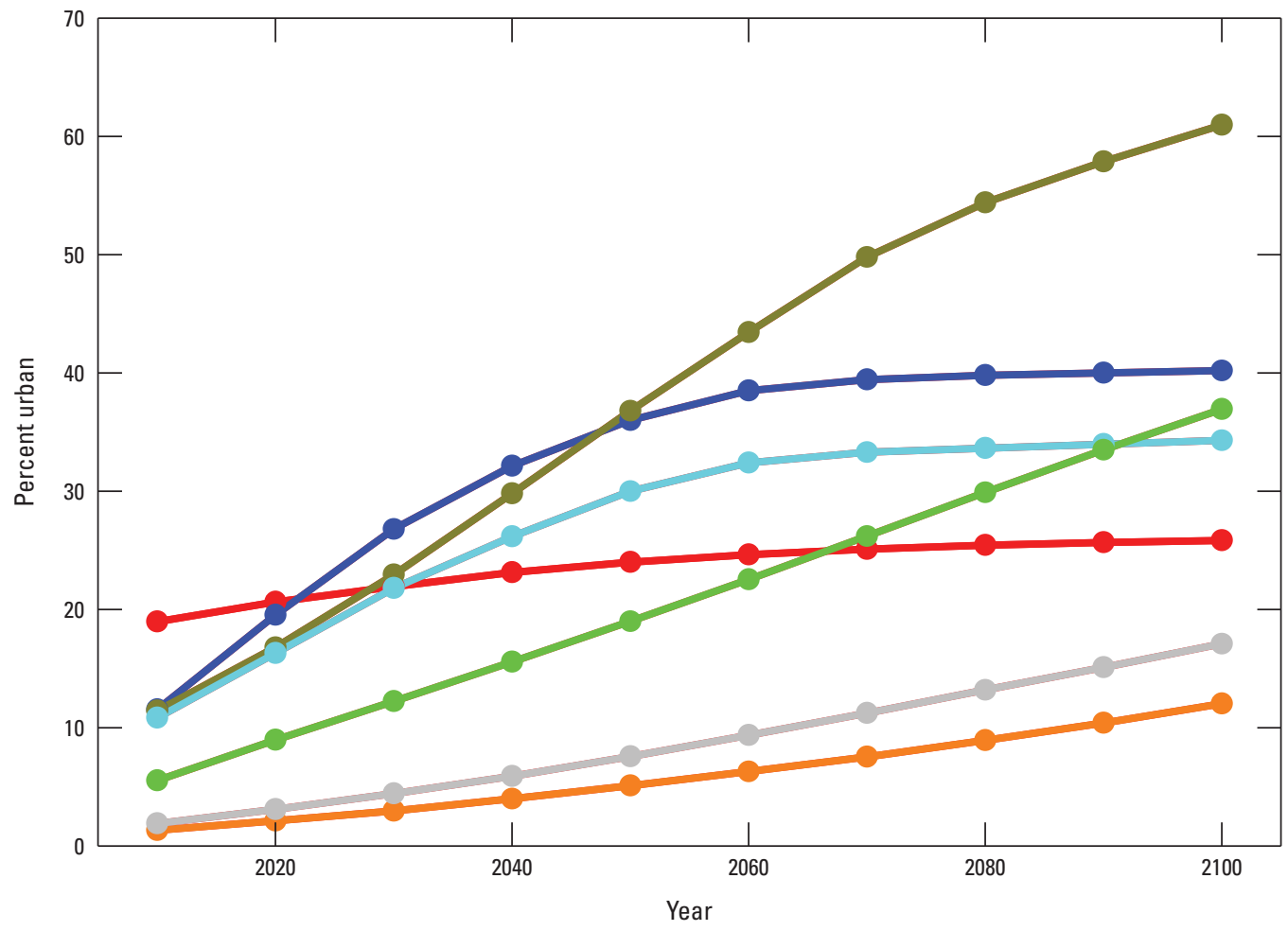

EXPLANATION

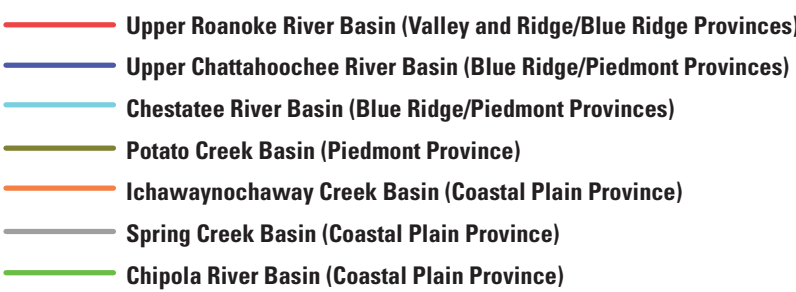

Figure 23. Trends in percent of land classified as urban for the seven fine-scale model basins from 2010 to 2100 (Belyea and Terando, 2012).

steepness on rates of soil erosion. The LS factor was calculated from a 30-m DEM following the methodology of Mitsova and others, 1996. The following equation represents the LS factor at a point, $r$, on a hillslope (Mitsova and others, 1996; Mitsova and Mitas, 1999).

$$
L S(r)=(m+1)\left[A(r) / a_{0}\right]^{m}\left[\sin b(r) / b_{0}\right]^{\mathrm{n}}
$$

where

$A \quad$ is the contributing area upslope per unit contour width, in meters;

$b \quad$ is the slope, in degrees;

$m$ and $n \quad$ are dimensionless parameters 0.6 and 1.3; and $a_{0}$ and $b_{0} \quad$ are the USLE plot standard length and slop values of $22.13 \mathrm{~m}$ and 9 percent or 5.16 degrees, respectively.
The equation above was parameterized as suggested by Mitsova and Mitas (2001) and entered in the ArcGIS map calculator using the 30-m DEM-derived flow accumulation and slope grids for the Apalachicola-Chattahoochee-Flint River Basin (fig. 24).

\section{Land Use}

The $C$ factor represents the effects of surface cover, roughness, biomass, and disruption activities on erosion (Renard and others, 1997). The $C$ factor is the ratio of soil loss from land under existing conditions to the corresponding soil loss from clean-tilled continuously fallow land under identical conditions, and is an average annual value (Wischmeier and Smith, 1978). An increase in $C$ factor represents an increase in erosion rate represented by a decrease in vegetative protection. Canopy cover, vegetative cover, and rooting structure are 
integrated in the $C$ factor. The $C$ factor was estimated from the 2001 National Landcover Dataset (NLCD) and Southeast GAP Analysis Project's Ecological System Classification descriptions using canopy cover and height estimates and the Natural Resources Conservation Service table of $C$ factors for permanent pasture, rangeland, idle land, and grazed woodlands (Renard and others, 1997; Homer and others, 2004; NatureServe, 2007) (fig. 24, appendix table 3-1).

\section{Soil Erosion Context}

Calculated soil erosion for the Apalachicola-Chattahoochee-Flint River Basin ranged from 0 to 200 tons per acre per year (tons/acre/year), equivalent to denudation rates of 0-15 millimeters per year ( $\mathrm{mm} / \mathrm{yr}$ ) (fig. 24). To assign values to classes, erosion rates from the literature in forested, geologic, and agricultural settings were examined (table 4) (Patric and others, 1984; Montgomery, 2007). Erosion rates of $0-0.25$ tons/acre/year $(0-0.02 \mathrm{~mm} / \mathrm{yr})$ were assigned as "low" and are characteristic of eastern forests and stable continental cratons in natural settings and within the low range of conservation agricultural practices (Patric and others, 1984; Montgomery, 2007). A medium class was assigned to erosion rates of $0.25-1$ tons/acre/year $(0.02-0.08 \mathrm{~mm} / \mathrm{yr})$ within the range of Eastern forests, moderate gradient hillslopes, and conservation agriculture. A high class was assigned to erosion rates greater than 1 ton/acre/year $(0.08 \mathrm{~mm} / \mathrm{yr})$. Mean rates of soil erosion per HRU also were calculated (fig. 25).

\section{Statistical Classification}

A clustering analysis was used for the reach-scale stream classification of the entire Apalachicola-Chattahoochee-Flint River Basin. Although reach- and segment-scale habitat variables were generated for the Upper Roanoke Basin for the metapopulation models, the stream classification was only generated for the Apalachicola-Chattahoochee-Flint River Basin. The main focus of the project was the ApalachicolaChattahoochee-Flint River Basin, and water temperature likely is the most important factor for trout persistence in the Upper Roanoke Basin. The classification contains 6 or 12 groups to provide a choice of resolution depending on the application of the classification. Statistical clustering analyses used Systat software version 11 (Systat Software, 2012).

\section{Cluster Analysis}

To minimize dependence among spatially adjacent stream reaches, a subset of approximately 20 percent of the stream reaches in the network $(20,217$ reaches out of 94,498$)$ was selected randomly for the statistical classification. This initial selection yielded a sample in which higher order streams (greater than the 5th Strahler order) were underrepresented. To ensure a more representative distribution of stream orders in the sample, 20 percent of the streams from the 1 st to the -4 th order were randomly selected and 50 percent of the streams from the 5 th to the 8 th order were selected randomly for initial clustering. This selection method does not necessarily remove adjacency effects, but the reduced number of features resulted in greater physical separation of stream reaches.

Exploratory statistical analyses on the selected dataset used drainage basin area, valley width, channel width, gradient, unit stream power, the ratio of valley width to channel width, and sinuosity as variables. A principal component analysis (fig. 26) indicated basin area, channel width, and valley width loading positively on factor 1 (or principal component 1). These variables all relate to basin size and there is a strong correlation between channel width and drainage basin area. This relation is expected because channel width was modeled as a function of basin area. Valley width also is correlated with drainage basin area and the ratio of valley width to channel width. Sinuosity was not indicated to be directly related to any other variables and is positively loaded on factor 3 . Sinuosity ultimately was judged inappropriate for use in the classification because it was generated from varying reach-length scales from a $30-\mathrm{m}$ DEM and is not necessarily reflective of true stream sinuosity. Gradient indicates a negative loading on factor 1, inverse to drainage basin area, which is expected because gradient at the scale of a large basin generally decreases with increases in drainage basin area. Stream power and gradient indicate positive loading of factor 2, suggesting this factor relates to stream energy. Scatterplots and histograms of the randomly selected stream reaches demonstrate relations between the variables and distributions (fig. 27).

Channel width, stream gradient, and valley width were selected as relatively independent variables for clustering and were standardized to their range. Channel width captures differences in stream size, which have been linked to ecosystem changes and aquatic communities (Vannote and others, 1980). Gradient captures differences in bedrock and surficial geology, stream size, and to some degree, land-use history. Valley width inherently captures differences in bedrock lithology and has been demonstrated to affect stream-channel form, such as the locations of riffles (White and others, 2010). These three variables were judged to be relatively independent of one another and indicative of stream characteristics. Scatterplots and histograms indicate the relations between these variables (fig. 28).

A K-means cluster analysis begins with a user-defined number of clusters $(K)$ and maximizes between-group variation to divide the data into $K$ mutually exclusive groups (Wilkinson and others, 2004). A K-means cluster analysis was run 19 times on the standardized, randomly selected dataset stipulating 2-20 clusters and an increment of 1 cluster. A plot of the between-group and among-group sum of square differences indicated a break at approximately 6 clusters and a flattening around 12 clusters (fig. 29). The clustering results from each run of the K-means classification also were examined (fig. 30). For instance, a small cluster of steep stream reaches in the Blue Ridge, represented by cluster 5 of the 6-cluster classification and cluster 12 of the 12-cluster classification, separated into two classes with just a few members 

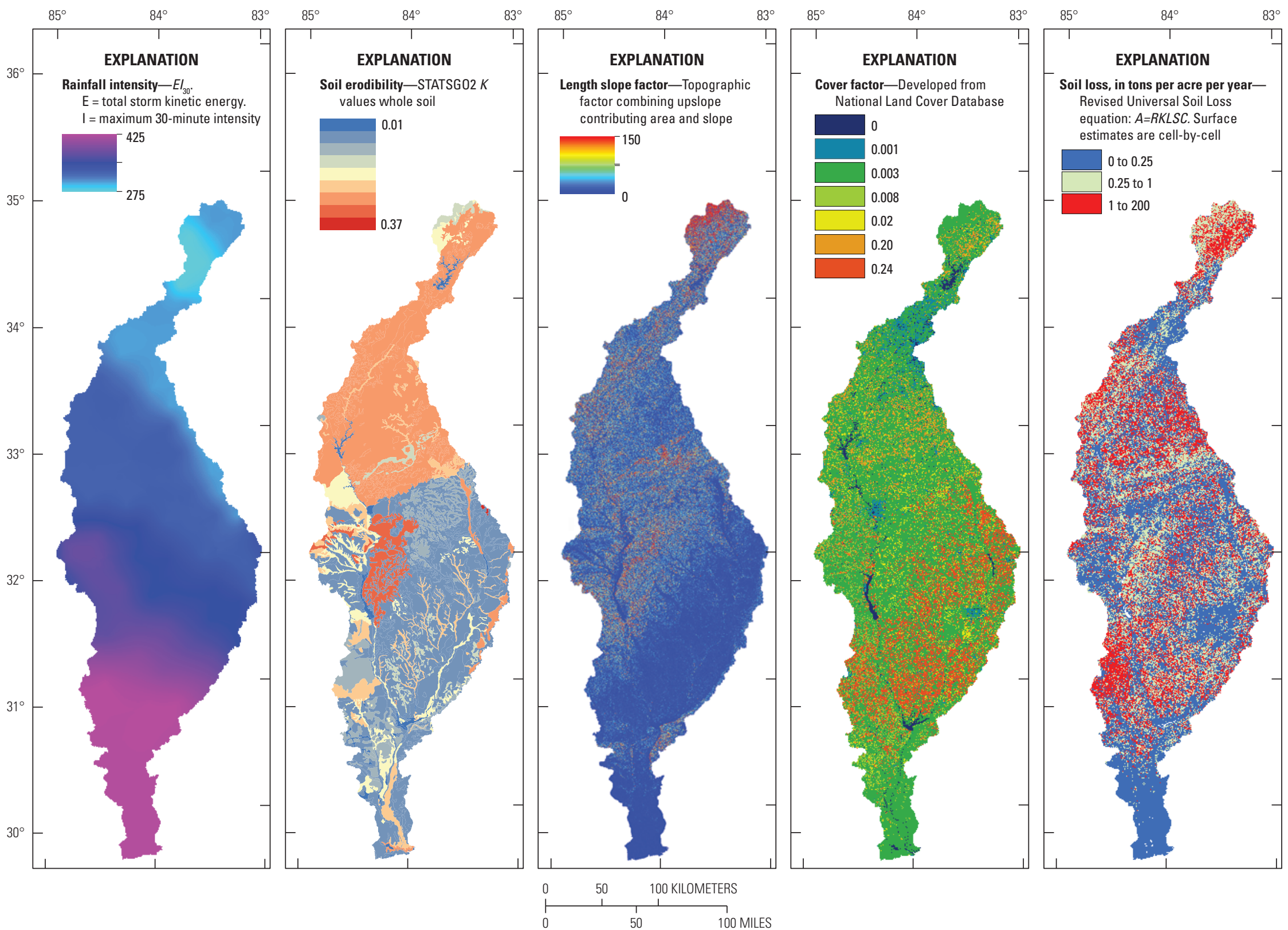

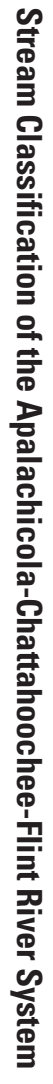

Figure 24. Revised Universal Soil Loss Equation (RUSLE) soil-erosion geographic information system model developed for the Apalachicola-Chattahoochee-Flint River Basin. 


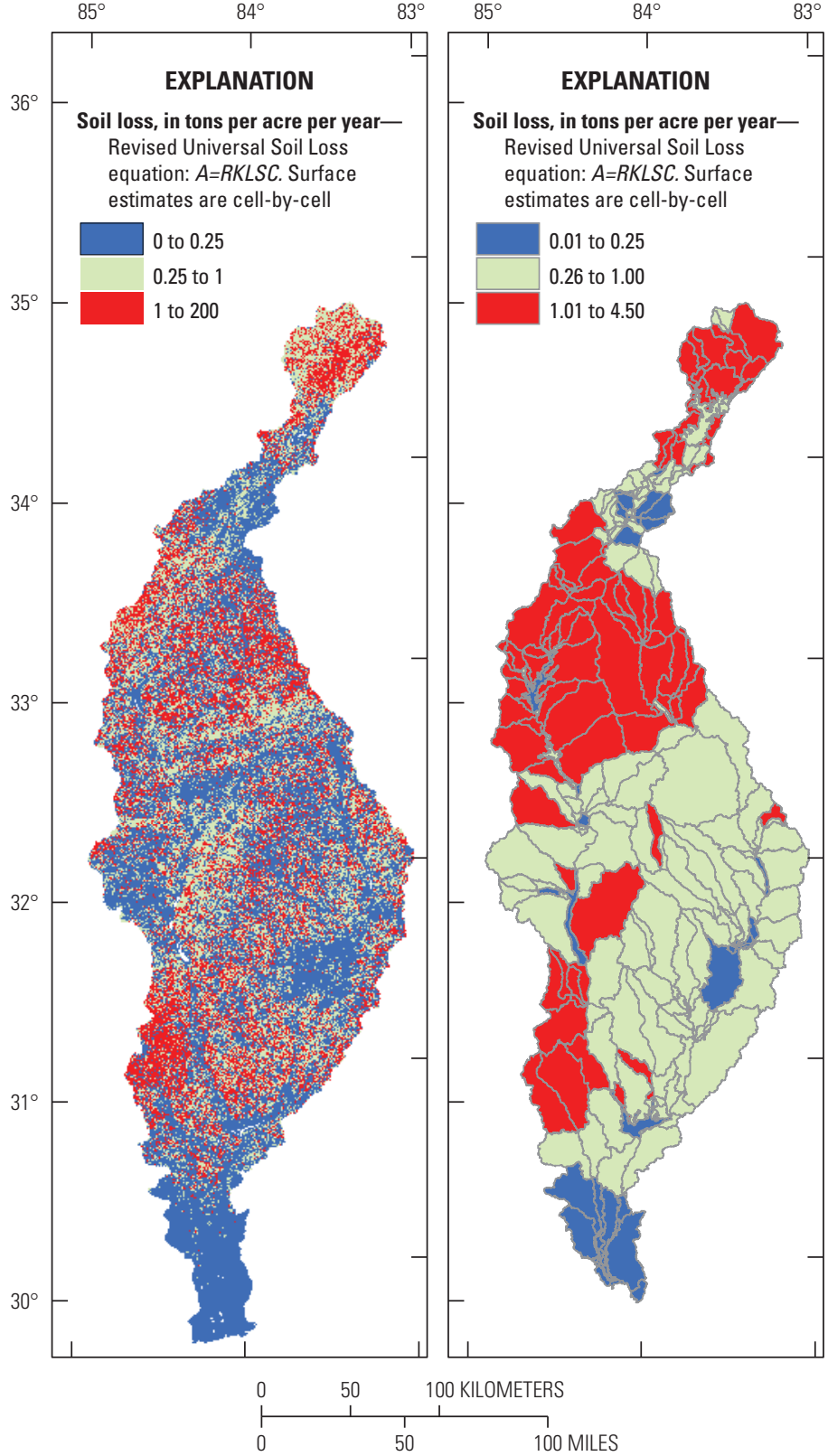

Figure 25. Revised Universal Soil Loss Equation (RUSLE) estimates of potential soil loss summarized by hydrologic response unit (HRU) in the Apalachicola-Chattahoochee-Flint River Basin.

at 16 groups. A classification that separated a cluster of a small number of streams into multiple groups was undesirable for a basin-wide classification. The K-means results also were joined back with their geospatial line segments in a GIS and the various clusterings were visualized spatially.

Ultimately, clusters produced based on two K values, 6 and 12 , were selected and discriminant analysis was used to determine the groupings for the entire dataset. Discriminant analysis plots indicate groupings from the clustering process

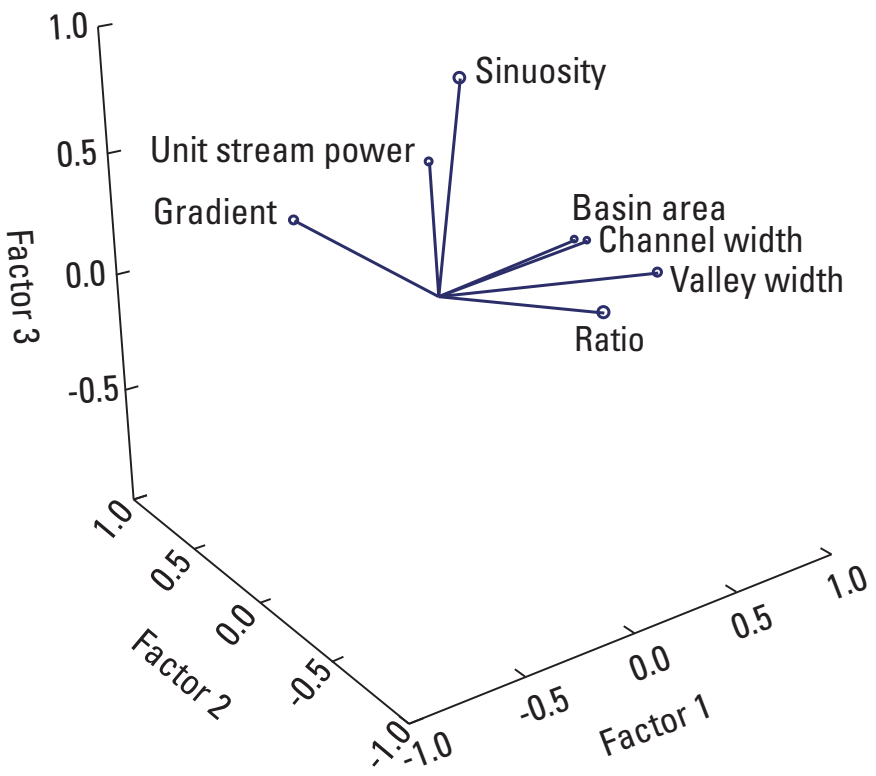

Figure 26. Principal component analysis (PCA) for seven geomorphic characteristics of a randomly selected subset of stream reaches in the Apalachicola-Chattahoochee-Flint River Basin.

and the relative sizes of the groups (figs. 31, 32). For example, in the 6-cluster classification, cluster 1 is dominant for the 6-cluster classification and cluster 5 is the smallest grouping but indicates the largest spread in cluster values because of the wide range of high gradients within this cluster with low channel and valley widths (fig. 31). There is some overlap in classification ellipses between clusters 6 and 4 in streams with intermediate channel and valley widths and low gradients (fig. 31). The classification was cross-validated by a jackknife procedure indicating that 92.7-100 percent of all reaches were classified correctly (table 5). Clusters 5 and 11 of the 12-cluster classification were misclassified, with the most misclassification being 7.3 and 3.8 percent, respectively. Both clusters predominately are composed of small streams with narrow valleys, with low gradients generally occurring in cluster 5 and low-to-medium gradients in cluster 11 (fig. 30). The rest of the classes were classified correctly 96.2100 percent of the time (table 5).

The final 6-cluster and 12-cluster classifications demonstrate the locations and distribution of classes across the Apalachicola-Chattahoochee-Flint River Basin and their relative sizes (figs. 33, 34). Box and whisker plots illustrate the distribution of the extended set of geomorphic variables in the 6- and 12-cluster classifications (figs. 35, 36, table 6). General cluster statistics are in table 6 and descriptions for individual clusters in table 7. With the exception of a few rare clusters, the field reconnaissance sites are representative of the 6-cluster and 12-cluster classifications (figs. 37, 38; table 6). 
Table 4. Measured erosion rates and ranges for native vegetation, geologic regions, and agricultural practices.

[tons/acre/year, tons per acre per year; mm/yr, millimeters per year; min, minimum; max, maximum; NRCS, Natural Resources Conservation Service; ,-- no data]

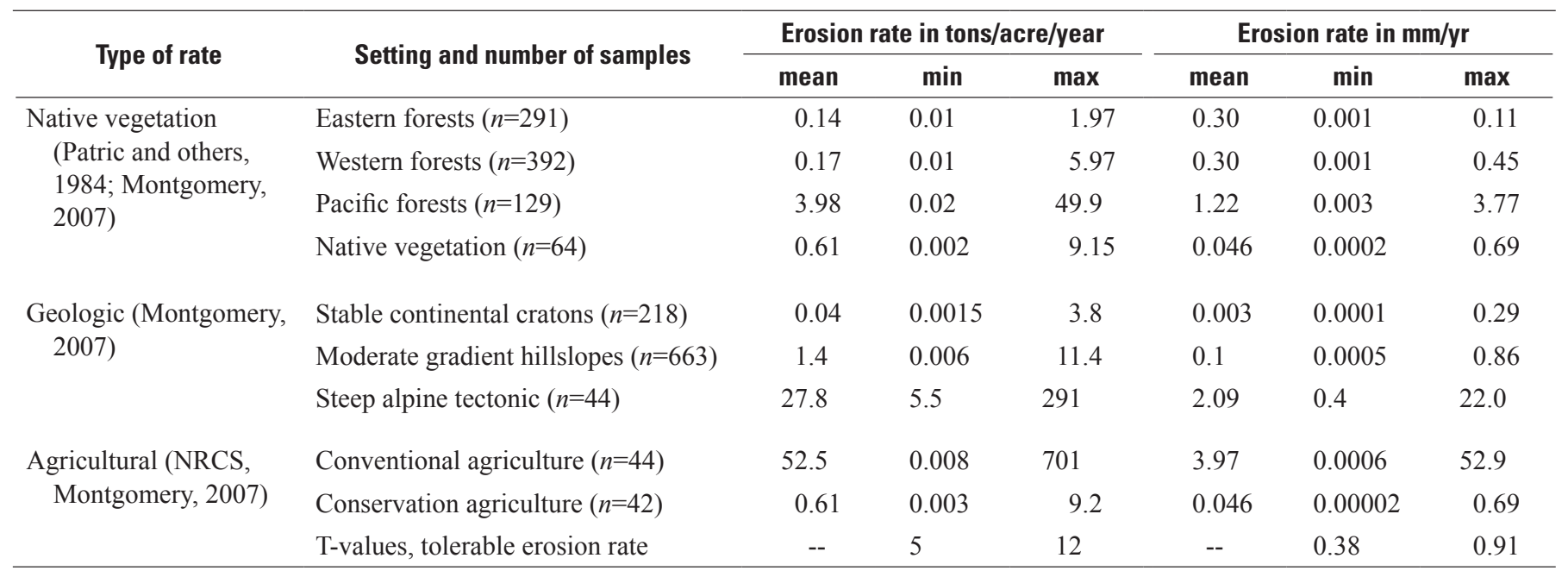

\section{Biological Validation of Stream Classification}

Stream classifications are useful if they can accurately predict outcomes of interest, which in this case extend from physical habitat to biotic responses, including the probability that a particular stream segment may contain species of interest. Stream geomorphology, especially measures of stream size, has been documented to be biologically relevant in predicting aquatic species distributions (Rieman and others, 2001; Wenger and others, 2008; Anderson and others, 2012) and other aspects of aquatic assemblages (Walters and others, 2003; Peterson and others, 2009). As one test of the biological relevance of the Apalachicola-Chattahoochee-Flint River Basin classification, we compared models using segment cluster assignments with models using individual geomorphic variables for predicting the occurrence of widely distributed species of fish within the Apalachicola-Chattahoochee-Flint River Basin.

\section{Species Evaluated}

Using data provided by the Georgia Museum of Natural History, we evaluated the relevance of the classification to observed occurrences of two species of fishes endemic to the Apalachicola-Chattahoochee-Flint River Basin. The Halloween darter (Percina crypta) recently was described as a new species endemic to the Apalachicola-ChattahoocheeFlint River Basin, where it lives in shoal and riffle habitats in four regions of the basin separated by main-stem reservoirs
(Freeman and others, 2008). The Halloween darter co-occurs with a closely related species, the blackbanded darter (Percina nigrofasciata), which is widely distributed in a range of stream habitats in southeast Atlantic Coast and Gulf Coast drainages (Crawford, 1956; Guillory, 1976; Freeman and others, 2008). Similarly, the bluestripe shiner (Cyprinella callitaenia) is known only from the Apalachicola-Chattahoochee-Flint River Basin, where it commonly co-occurs with the blacktail shiner (Cyprinella venusta), a species with a broad distribution in Gulf Coast drainages from Georgia and Florida to the lower Mississippi Basin and Texas (Warren and others, 2000). Datasets comprising all collection records of these darter and minnow species in the Apalachicola-Chattahoochee-Flint River Basin were used to (1) evaluate the ability of the ApalachicolaChattahoochee-Flint River Basin stream classification to predict occurrences of the endemic species, and (2) tabulate the occurrences of each species in relation to prevalence of stream classes in the datasets of sampled streams. For each dataset, observed fish locations were joined spatially to the classified and attributed stream network to associate each fish location with the stream-network, classification unit, and associated habitat values. Fish locations had varying degrees of spatial accuracy and were excluded from the analysis if they fell too far away from the stream network to be assigned to a stream segment or intersected with layers identifying waterbodies and reservoirs. 


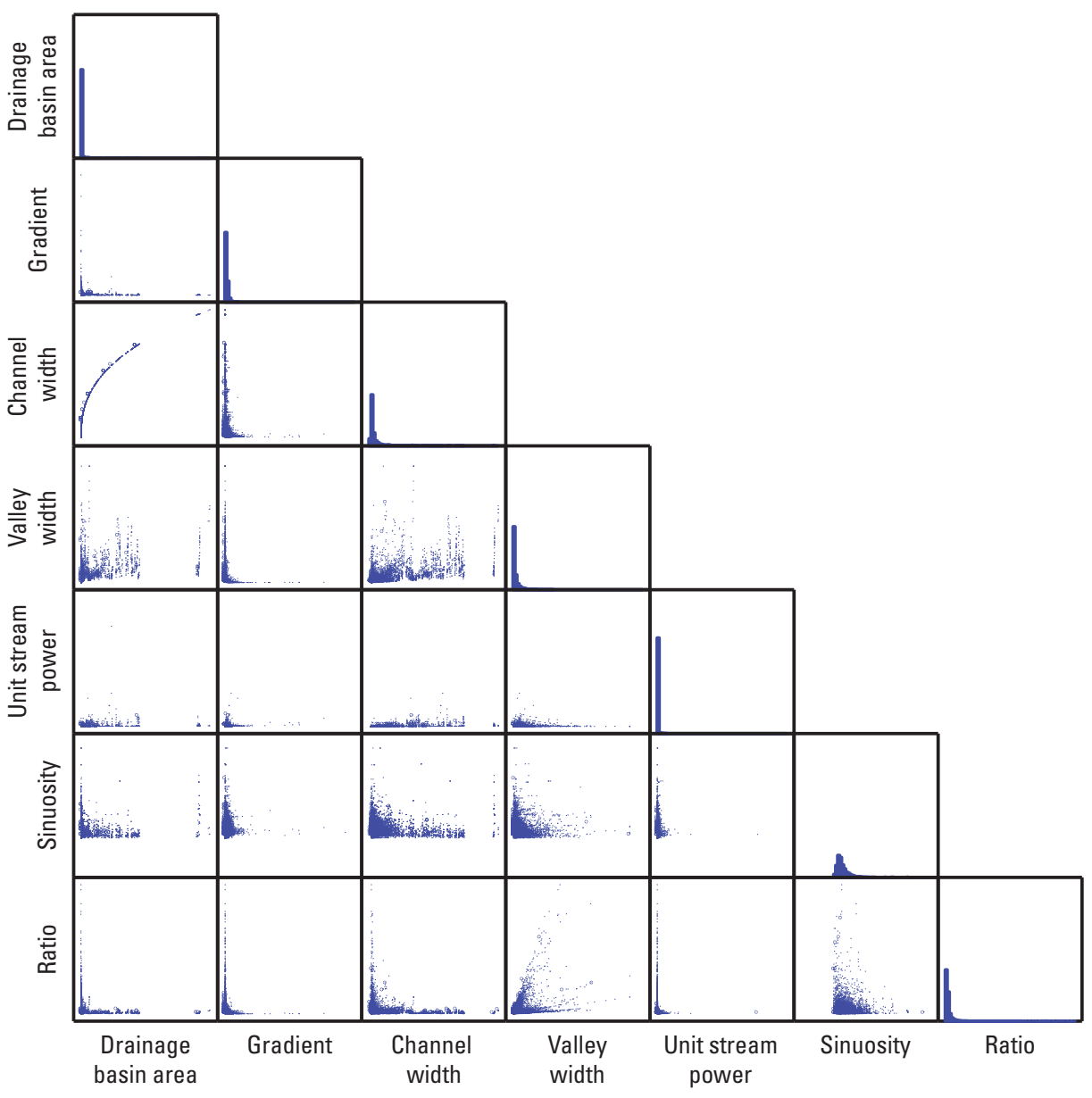

Figure 27. Scatterplots and histograms of seven geomorphic characteristics for a randomly selected subset of stream reaches in the Apalachicola-Chattahoochee-Flint River Basin.

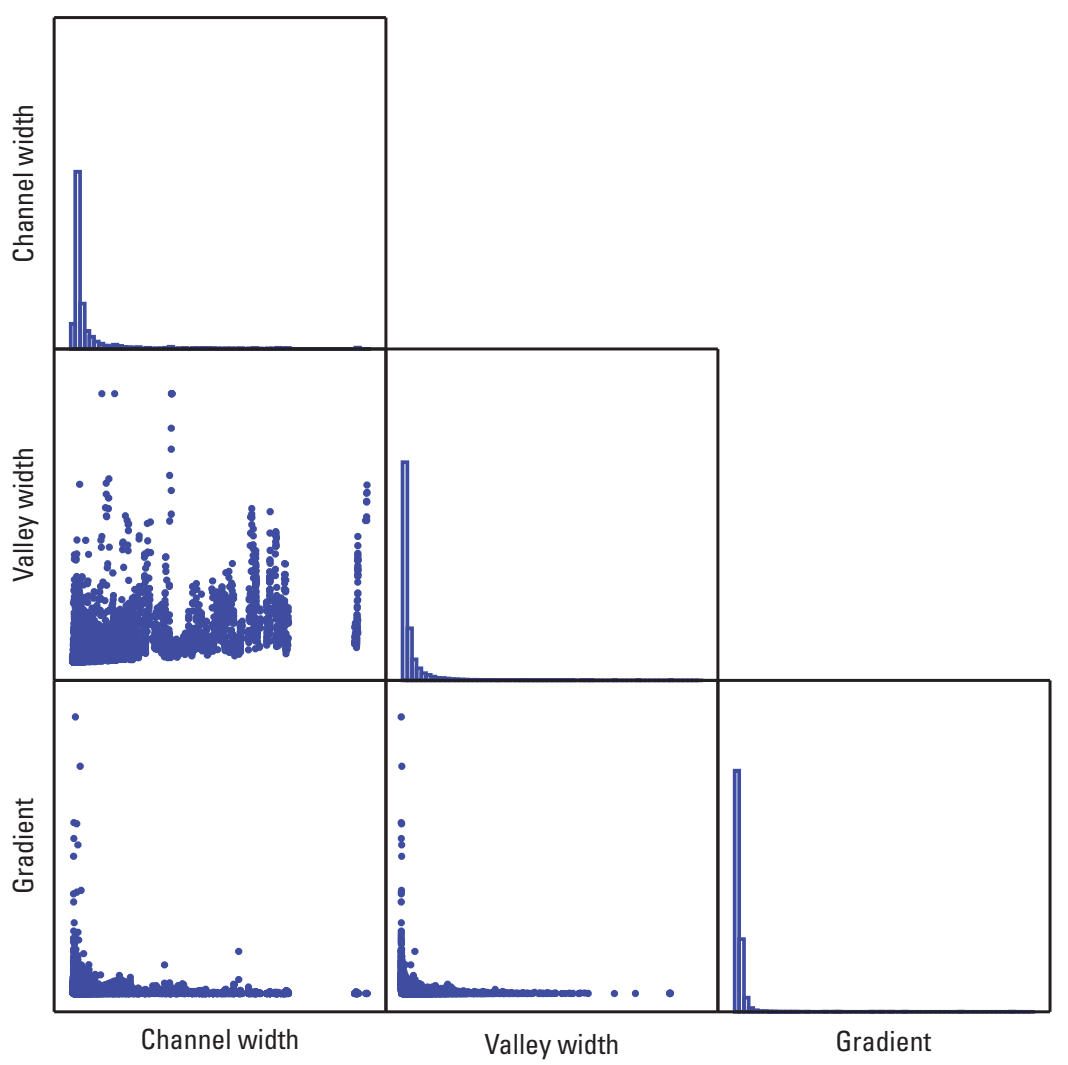

Figure 28. Scatterplots and histograms for channel width, valley width, and gradient measured at a randomly selected subset of stream reaches in the ApalachicolaChattahoochee-Flint River Basin. 


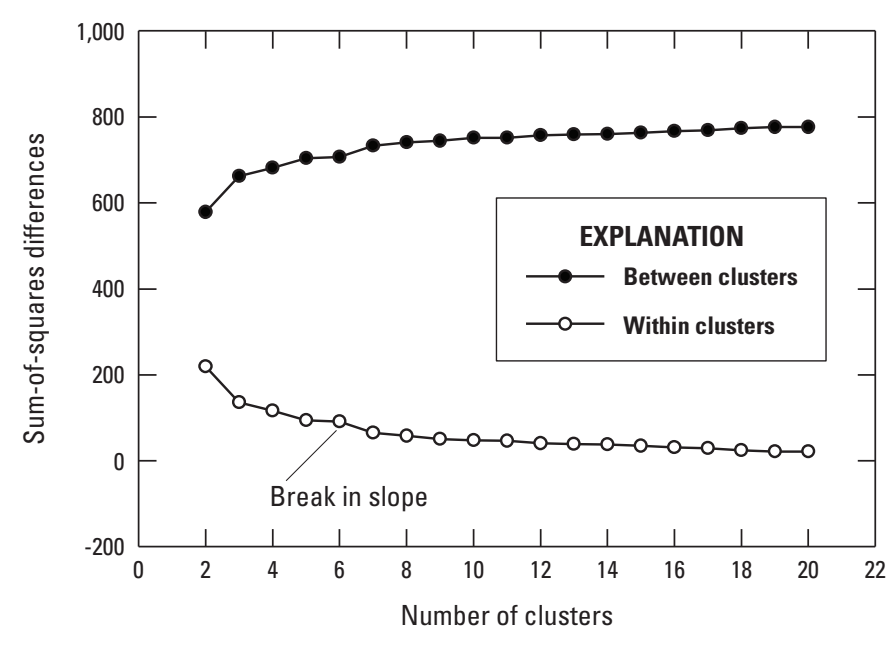

Figure 29. Changes in the sum-of-squares differences within and between clusters as number of clusters changes.

\section{Species Occurrence Model Testing}

Distribution data for the two darter species were available for 734 sites. Six observed fish locations were excluded because they fell outside the Apalachicola-ChattahoocheeFlint River Basin, and 21 additional fish locations were excluded from analysis because they fell on stream segments identified as lake waterbodies, which are not included in the classification scheme. All excluded locations were sites where only the blackbanded darter was observed. The Halloween darter was observed at 51 of the 707 fish locations used in the analysis, whereas the blackbanded darter was observed at all locations. Distribution data for the two minnow species comprised 378 sample locations in the ApalachicolaChattahoochee-Flint River Basin, of which 21 locations were excluded because they either could not easily be associated with a stream in the DEM-generated network or were located in major reservoirs. The bluestripe shiner was observed at 77 of the remaining 357 sample locations, whereas the blacktail shiner was observed at 349 sites.

Geomorphic variables were extracted for the segment containing each observed fish location along with the class assignment (based on the 6-cluster classification scheme) for each segment. Logistic regression models were used with either geomorphic variables or class assignment as predictors of observed occurrence of the endemic species (the Halloween darter or the bluestripe shiner) within the darter and minnow datasets. For geomorphic predictors, we selected four weakly correlated [Pearson $r$ is less than 0.4 ] variables related to factors hypothesized as relevant to instream habitat: stream size (drainage area, in square kilometers), stream-channel slope (mean segment gradient), channel confinement (ratio of valley width to channel width), and planimetric form (segment sinuosity). Geomorphic predictors were standardized by subtracting the mean and dividing by the standard deviation for each. Model Akaike Information Criterion (AIC) values were used to compare support among these models and with regressions using stream class as a predictor. AIC provides a relative measure of the distance between fitted model and the unknown true mechanism underlying the observed data, with smaller values reflecting better models (Burnham and Anderson, 2002). Models with AIC values that differed by more than 10 compared to the AIC of the best-supported model were considered to have essentially no support (Burnham and Anderson, 2002).

Prediction accuracy between the best-supported AIC models based on geomorphic variables and on stream classes was compared by using leave-one-out cross-validation. Probability of endemic species occurrence was predicted for each observation in the dataset using regression coefficients from the best-supported geomorphic variable or stream class model fit to all remaining observations. Commission error rate was calculated as the proportion of predicted presences (that is, a predicted probability of 0.5 or greater) where the endemic species was not observed. Omission error rate was the proportion of observations of the endemic species where predicted probability of occurrence was less than 0.5 ("predicted absence"). All analyses used the statistical software platform R (R Development Core Team, 2009).

The best-supported model using geomorphic variables for observed occurrence of the Halloween darter included all assessed variables (drainage area, mean gradient, segment sinuosity, and ratio of valley width to channel width; table 8). The best-supported model based on the stream classification included segment assignment to either (or none) of two clusters (clusters 3 and 4 , table 8 ). Both models were substantially better-supported than an intercept-only model (table 8). Regression coefficient estimates mostly were far from zero for both models (table 9). However, modeling Halloween darter occurrence using segment classification generally resulted in much lower model deviance and AIC values compared to models using geomorphic variables (table 8 ). Prediction errors (based on leave-one-out cross-validation) were $<0.4$ using the classification model (table 10), with Halloween darters predominantly in segments assigned to cluster 3 (table 11). In contrast, the model based on individual geomorphic variables failed almost completely in predicting Halloween darter occurrence (table 10). Thus, despite having relatively high support compared to the null model and reasonably precise parameter estimates, the geomorphic variable model had little predictive utility for this fish species.

For the bluestripe shiner, the best-supported geomorphic and classification-based models had similar support based on model AIC values (table 8). The best-supported geomorphic model included drainage area and a quadratic term for drainage area (indicating higher occurrence rates in medium-sized streams), ratio of valley width to channel width, and segment sinuosity. Predicted occurrence of the bluestripe shiner was highest in segments with mid-sized drainage area and narrower valley width relative to channel width (table 9). Using stream clusters, the best-supported predictive model included 
A. 6-Cluster classification

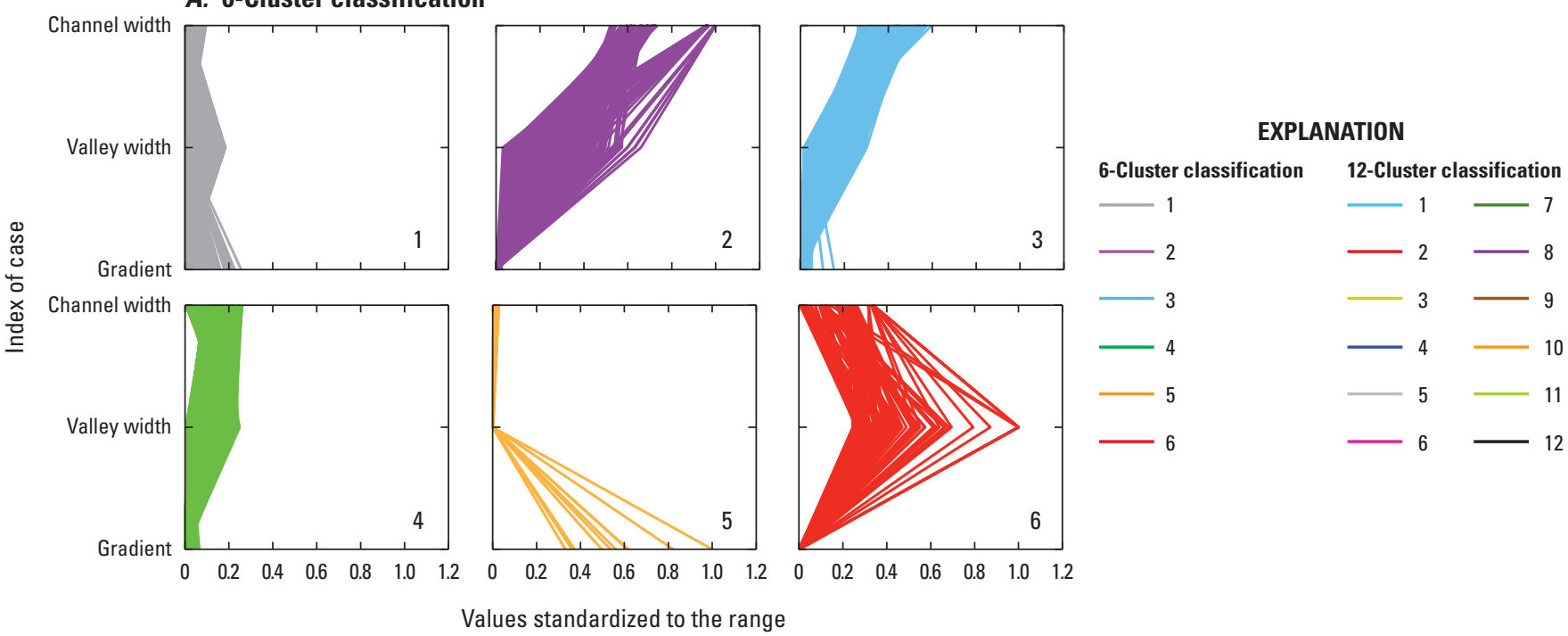

\section{B. 12-Cluster classification}
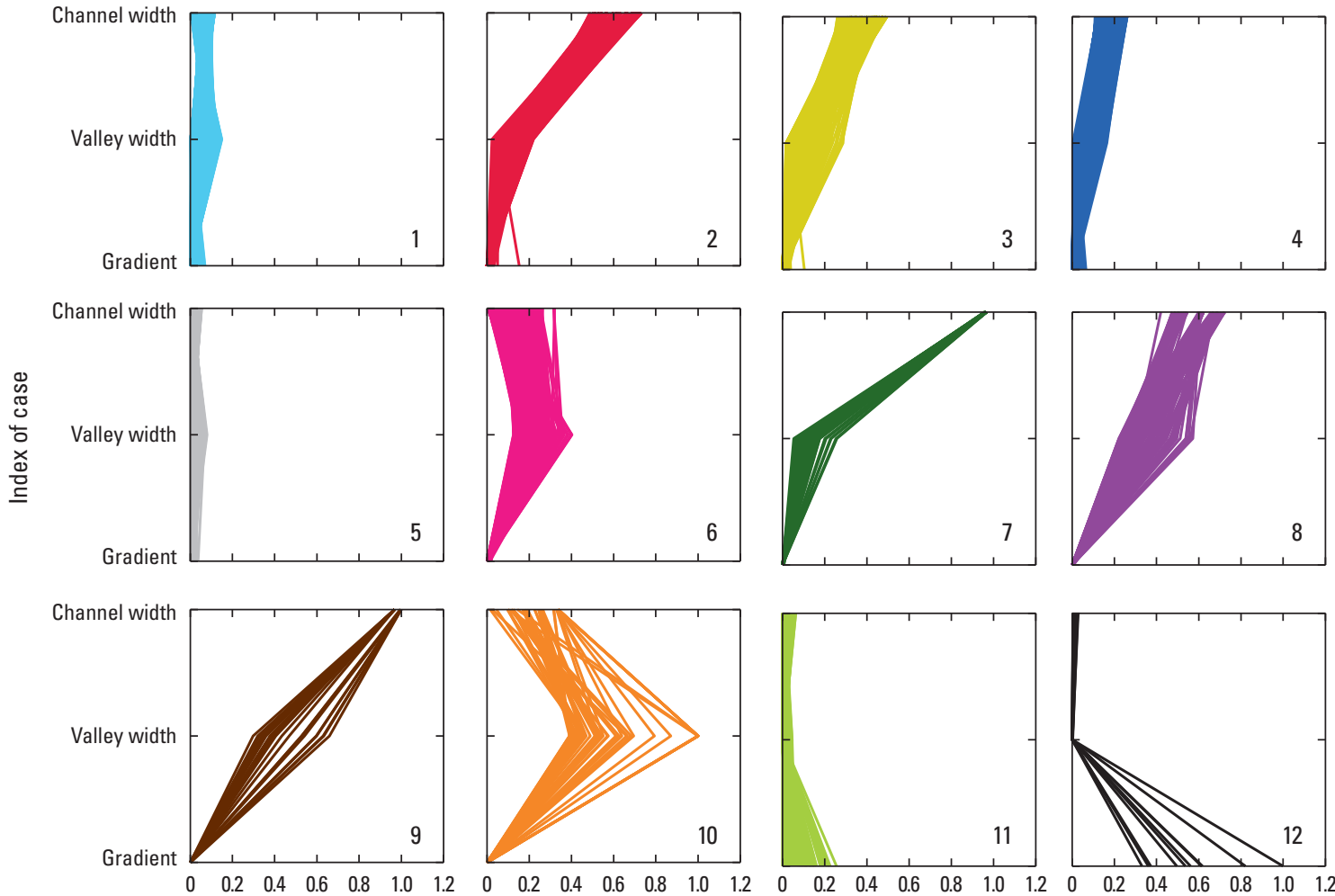

Values standardized to the range

Figure 30. Clustering results from the K-means procedure at 6 and 12 clusters. The variables show standardized values of the variables (relative to the mean) for each cluster andare arranged from most to least effect from top to bottom. 
Figure 31. Canonical scores for the 6-cluster classification of stream reaches within the ApalachicolaChattahoochee-Flint River Basin. Ellipses show the groupings used for the classification.

Figure 32. Canonical scores for the 12-cluster classification of stream reaches within the ApalachicolaChattahoochee-Flint River Basin. Ellipses show the groupings used for the classification.

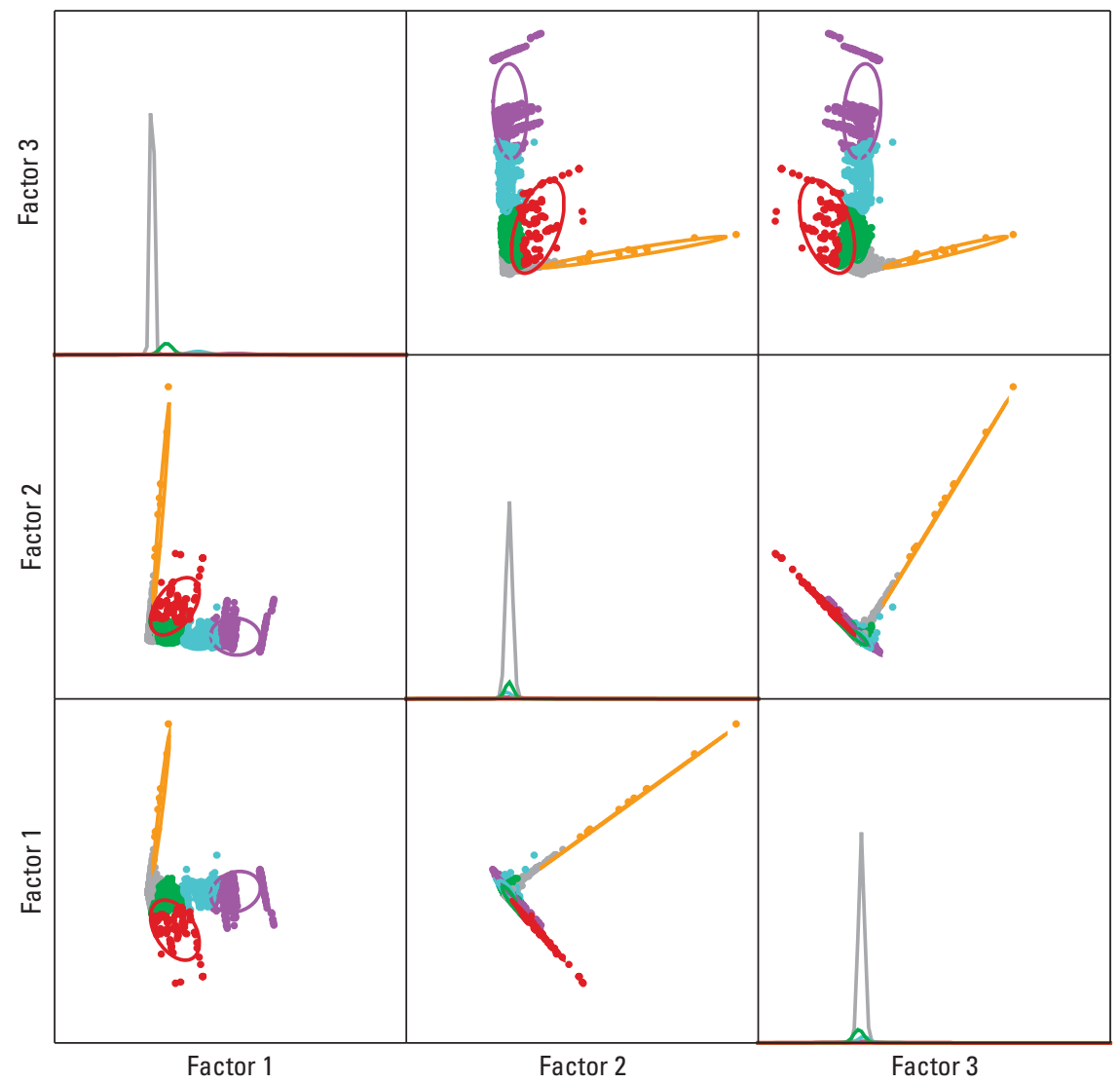

EXPLANATION

Cluster number

- 1

- 2

- 3

- 4

- 5

- 6

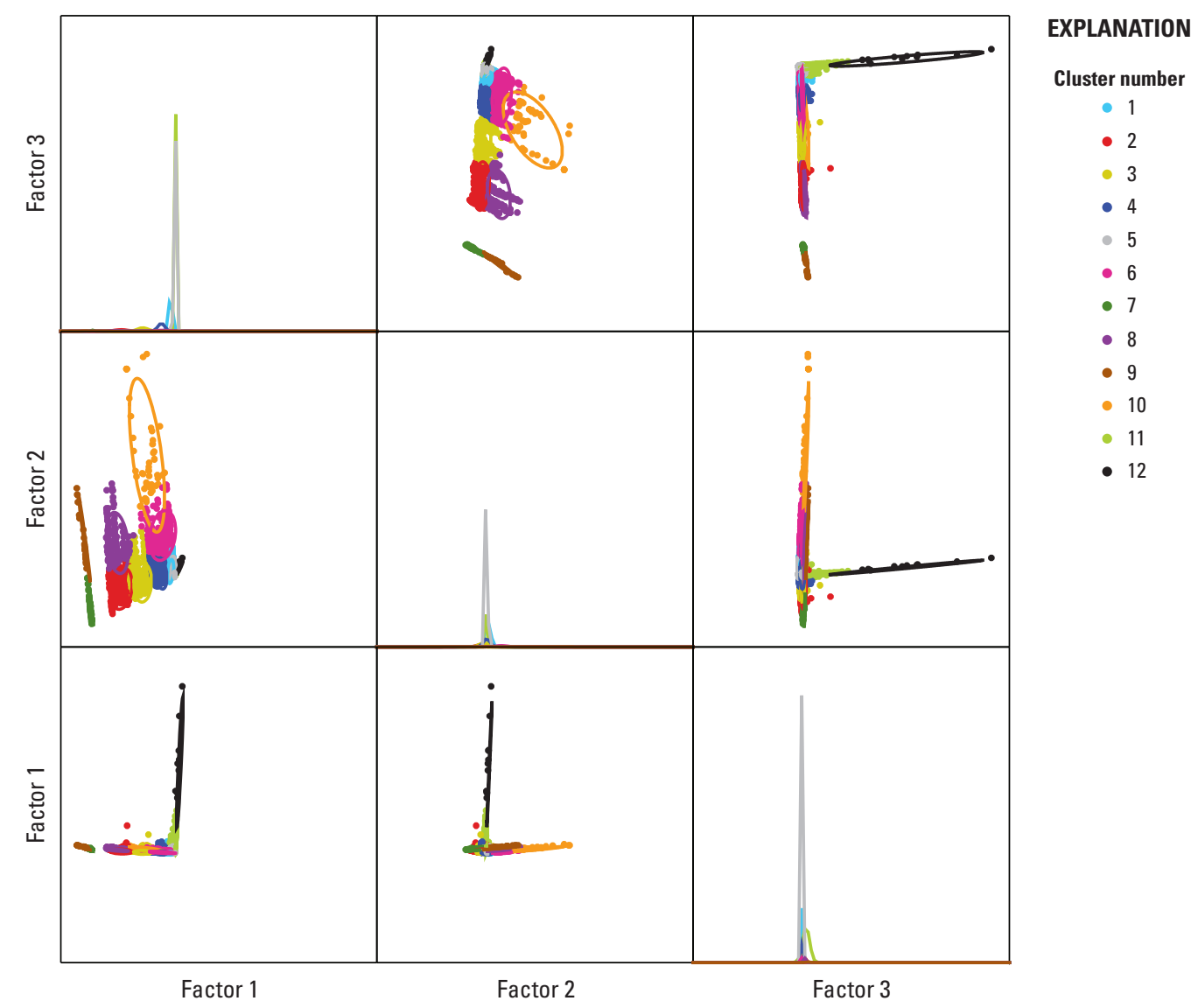


segments in clusters 2, 3, or 4 (table 9), or medium- to largesized streams with relatively narrow valleys. Prediction error rates were similar for the two models, with commission error rates less than 0.3 and omission error rates between $0.5-0.6$ (table 10). For endemic species, prediction-error rates were higher for false negatives.

\section{Species Occurrence in the 6- and 12-Cluster Classifications}

Collection records for the distribution of blackbanded and Halloween darters in the 6- and 12-cluster classifications are indicated in table 12. In the 6-cluster classification, cluster 1 is associated with 76.2 percent of the locations where only the blackbanded darter occurs (table 12, fig. 39). When all blackbanded darter locations are considered, cluster 1 (71.7 percent) and cluster 4 (21.8 percent) account for nearly all of the locations. These clusters are associated with small streams (low drainage basin areas and channel width) for cluster 1 and medium streams (intermediate drainage basin areas and channel widths) for cluster 4 (table 12, fig. 39). Clusters 1 and 4 also represent the most dominant streams in the Apalachicola-Chattahoochee-Flint River Basin (89.9 and 6.8 percent of all reaches, respectively, table 12). Halloween darters are found most frequently in streams belonging to clusters 3 and 4 (60.8 percent 25.5 percent respectively, table 12$)$. Cluster 3 predominantly is made up of medium to large main-stem rivers, but excludes the largest streams in the basin and represents a low percent (1.2 percent, table 12) of streams in the Apalachicola-Chattahoochee-Flint River Basin. In the 12-cluster classification, the blackbanded darter dominantly occurs in clusters 1 and 5 (41.4 and 30.7 percent respectively, fig. 40, table 12). These clusters also are two of the dominant three clusters overall in the Apalachicola-Chattahoochee-Flint River Basin, accounting for 92.8 percent of all stream reaches representing small-to-medium sized streams (fig. 40, tables 6, $7,12)$. The Halloween darter occurs predominantly in clusters 3 and 4 (54.9 and 17.6 percent, respectively, table 12). These clusters represent medium sized streams with intermediate gradients, relatively high unit stream power, and relatively narrow valleys including the bedrock-dominated shoal reaches of the Flint River and Ichawaynochaway Creek (figs. 39, 41; tables 6, 7).

The distribution of the two minnows, the bluestripe and blacktail shiner were also compared to the 6- and 12-cluster classification (figs. 41, 42, table 12) The blacktail shiner is associated with clusters 1 and 4 (57.9 and 36.1 percent, respectively, table 12) of the 6-cluster classification. The two clusters ( 1 and 4) that the blacktail shiner is dominant in are also the two dominant clusters in the Apalachicola-ChattahoocheeFlint River Basin (76.2 and 21.5 percent, respectively). The bluestripe shiner is predominantly in clusters 3 and 4 with 46.8 percent in cluster 3 and 32.5 percent in cluster 4 (table 12). Cluster 3 stream reaches comprise 1.8 percent of streams in the Apalachicola-Chattahoochee-Flint River Basin and generally are medium-sized main-stem rivers (tables 6, 7). The blacktail shiner is present predominantly in cluster 1 of the 12-cluster classification (55.7 percent), with lesser percentages in clusters 4 and 5(15.7 and 16.4 percent, respectively). The bluestripe shiner is documented predominantly in clusters 1,3 , and 4 of the 12-cluster classification (19.5, 45.5, and 24.7 percent, respectively). Clusters 3 and 4 generally are rare with 1.4 and 3.1 percent of streams in the ApalachicolaChattahoochee-Flint River Basin.

Both endemic species (the Halloween darter and bluestripe shiner) are present primarily in restricted reaches that account for only 17.9 percent of the Apalachicola-Chattahoochee-Flint River Basin total - that is, less than 18 percent of the Apalachicola-Chattahoochee-Flint River Basin reaches account for 84.3 percent of Halloween darter occurrences and 89.7 percent of bluestripe shiner occurrences (table 12). In contrast, the broadly distributed species are found in reach clusters that account for 92.8 percent (blackbanded darter) and 73.2 percent (blacktail shiner) of the ApalachicolaChattahoochee-Flint River Basin reaches. 
Table 5. Jackknife validation for the 6- and 12-cluster classifications of streams in the Apalachicola-Chattahoochee-Flint River Basin. $[--$, no data $]$

\begin{tabular}{|c|c|c|c|c|c|c|c|c|}
\hline & \multirow{2}{*}{ Cluster } & \multicolumn{6}{|c|}{ Cluster } & \multirow{2}{*}{$\begin{array}{l}\text { Percent } \\
\text { correct }\end{array}$} \\
\hline & & 1 & 2 & 3 & 4 & 5 & 6 & \\
\hline & \multicolumn{8}{|c|}{ 6-Cluster classification } \\
\hline & 1 & 16,943 & -- & -- & -- & -- & -- & 99.7 \\
\hline & 2 & -- & 469 & -- & -- & -- & -- & 98.7 \\
\hline \multirow{5}{*}{$\begin{array}{c}\text { Classified by } \\
\text { jackknife }\end{array}$} & 3 & -- & -- & 846 & -- & -- & -- & 99.6 \\
\hline & 4 & -- & -- & -- & 1,734 & -- & -- & 97.7 \\
\hline & 5 & -- & -- & -- & -- & 11 & -- & 100.0 \\
\hline & 6 & -- & -- & -- & -- & -- & 110 & 98.2 \\
\hline & Total & 16,996 & 475 & 849 & 1,774 & 11 & 112 & 99.0 \\
\hline
\end{tabular}

\begin{tabular}{|c|c|c|c|c|c|c|c|c|c|c|c|c|c|c|}
\hline & \multirow{2}{*}{ Cluster } & \multicolumn{12}{|c|}{ Cluster } & \multirow{2}{*}{$\begin{array}{l}\text { Percent } \\
\text { correct }\end{array}$} \\
\hline & & 1 & 2 & 3 & 4 & 5 & 6 & 7 & 8 & 9 & 10 & 11 & 12 & \\
\hline & \multicolumn{14}{|c|}{ 12-Cluster classification } \\
\hline & 1 & 2,489 & -- & -- & -- & -- & -- & -- & -- & -- & -- & -- & -- & 98.8 \\
\hline & 2 & -- & 402 & -- & -- & -- & -- & -- & -- & -- & -- & -- & -- & 100.0 \\
\hline & 3 & -- & -- & 683 & -- & -- & -- & -- & -- & -- & -- & -- & -- & 99.6 \\
\hline & 4 & -- & -- & -- & 1,018 & -- & -- & -- & -- & -- & -- & -- & -- & 99.2 \\
\hline & 5 & -- & -- & -- & -- & 10,037 & -- & -- & -- & -- & -- & -- & -- & 92.7 \\
\hline \multirow{8}{*}{$\begin{array}{l}\text { Classified by } \\
\text { jackknife }\end{array}$} & 6 & -- & -- & -- & -- & -- & 258 & -- & -- & -- & -- & -- & -- & 98.1 \\
\hline & 7 & -- & -- & -- & -- & -- & -- & 61 & -- & -- & -- & -- & -- & 100.0 \\
\hline & 8 & -- & -- & -- & -- & -- & -- & -- & 149 & -- & -- & -- & -- & 98.7 \\
\hline & 9 & -- & -- & -- & -- & -- & -- & -- & -- & 26 & -- & -- & -- & 100.0 \\
\hline & 10 & -- & -- & -- & -- & -- & -- & -- & -- & -- & 51 & -- & -- & 100.0 \\
\hline & 11 & -- & -- & -- & -- & -- & -- & -- & -- & -- & -- & 4,036 & -- & 96.2 \\
\hline & 12 & -- & -- & -- & -- & -- & -- & -- & -- & -- & -- & -- & -- & 100 \\
\hline & Total & 2,519 & 402 & 686 & 1,026 & 10,824 & 263 & 61 & 151 & 26 & 51 & 4,197 & 11 & 98.6 \\
\hline
\end{tabular}



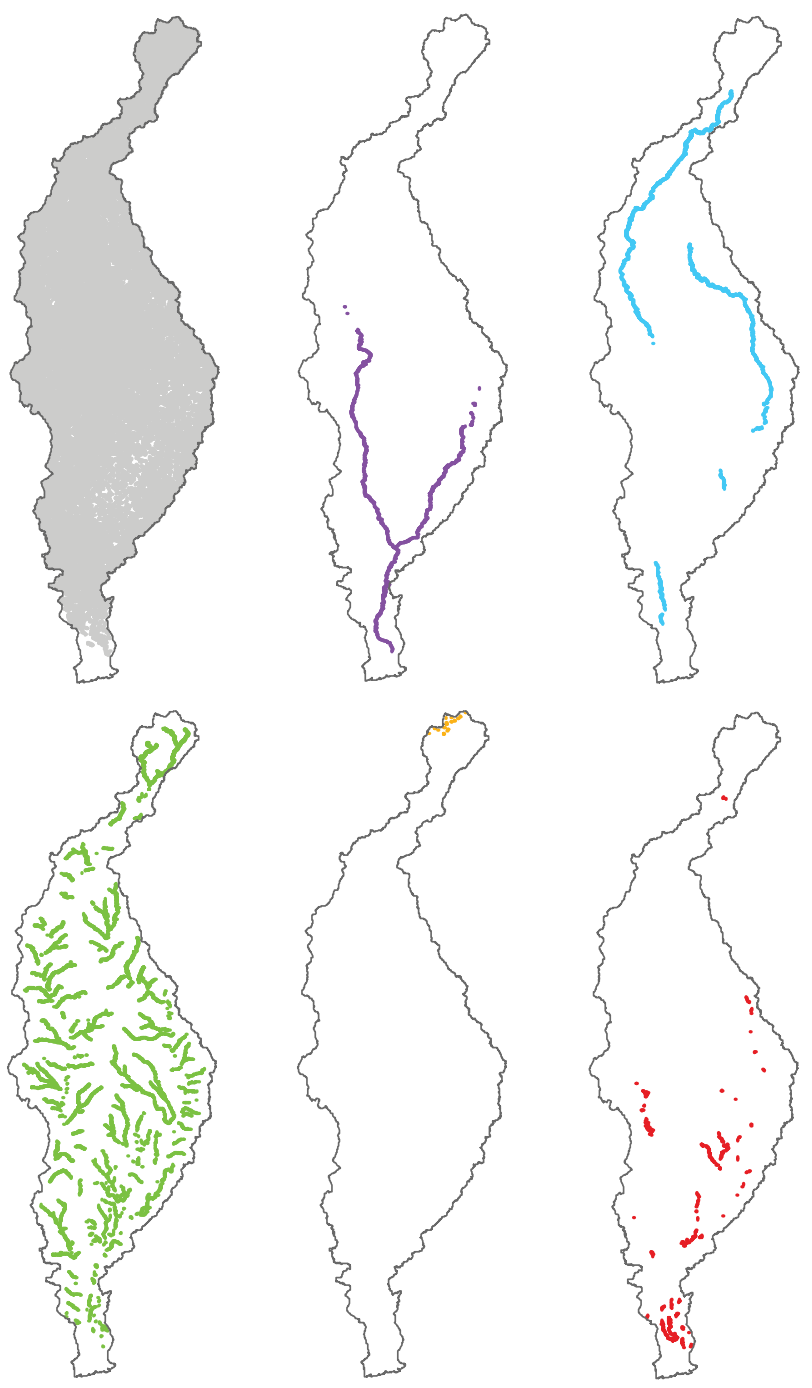

EXPLANATION

6-Cluster classification

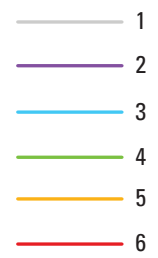

Figure 33. Spatial distribution of stream reaches within the Apalachicola-Chattahoochee-Flint River Basin for the 6 -cluster classification. 

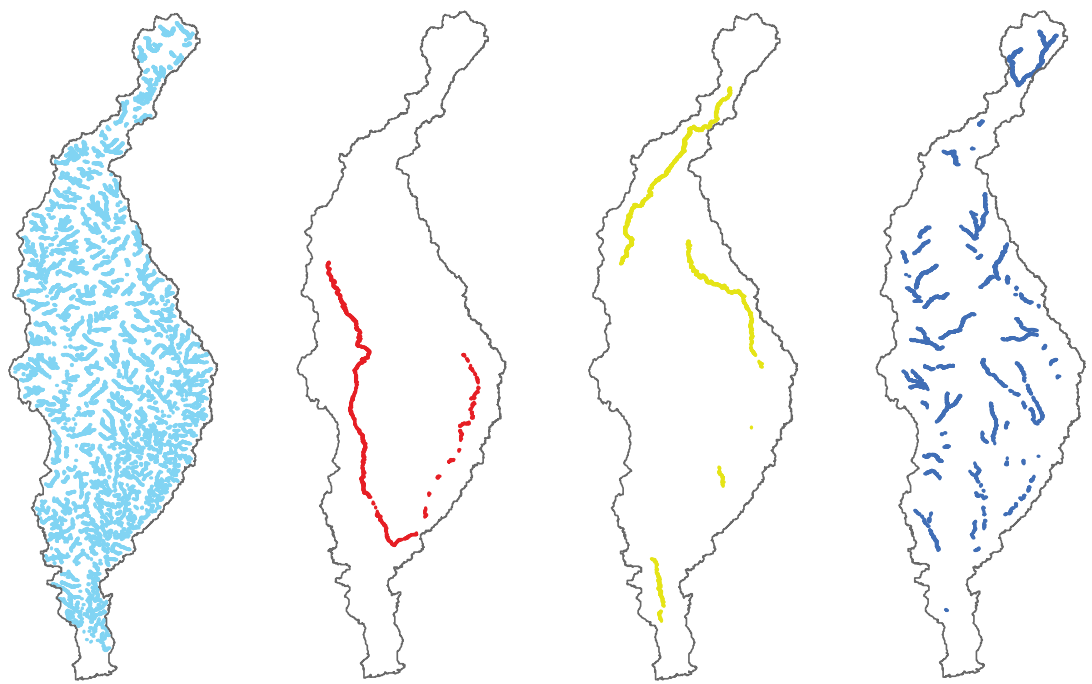

EXPLANATION
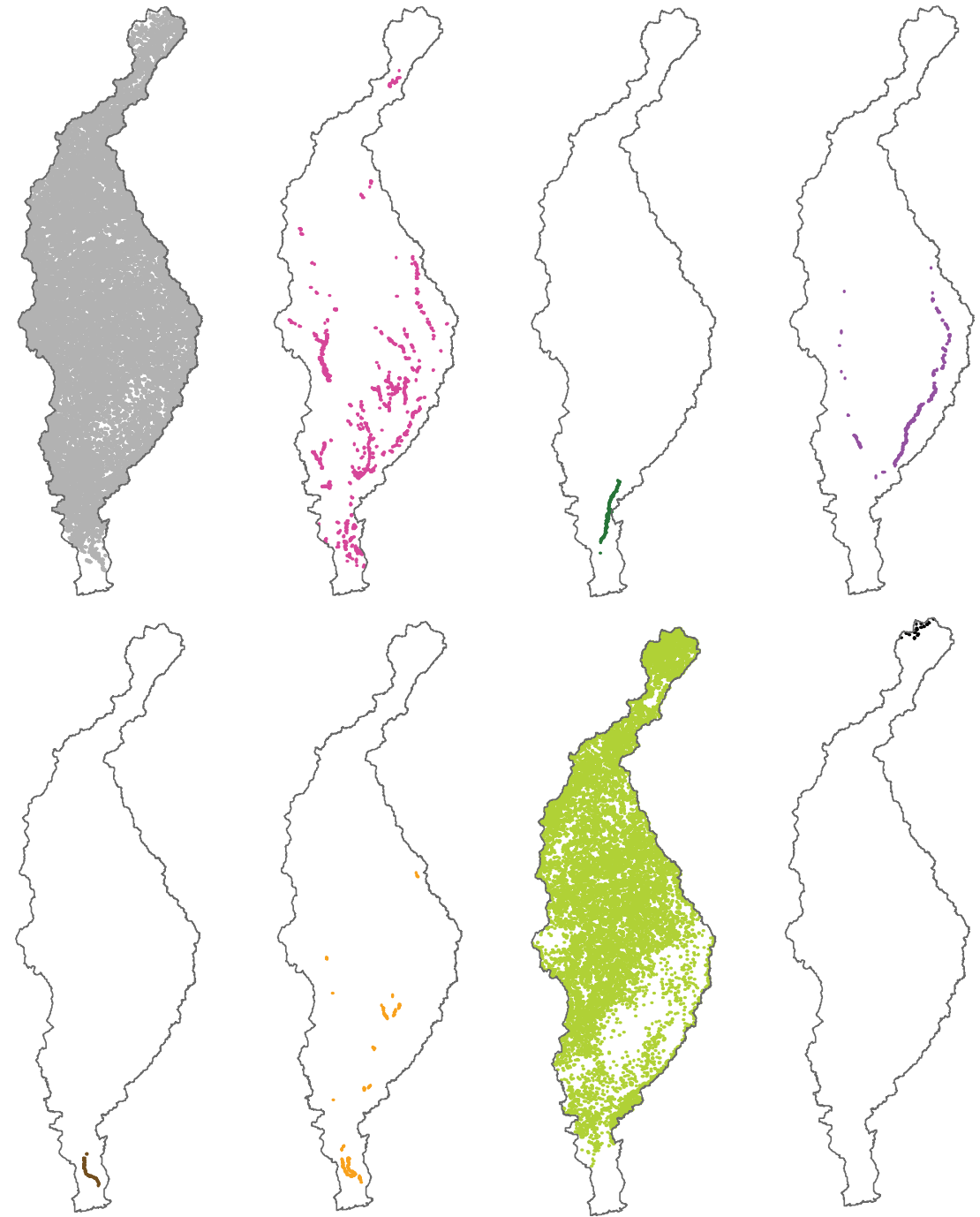

Figure 34. Spatial distribution of stream reaches within the Apalachicola-Chattahoochee-Flint River Basin for the 12-cluster classification. 

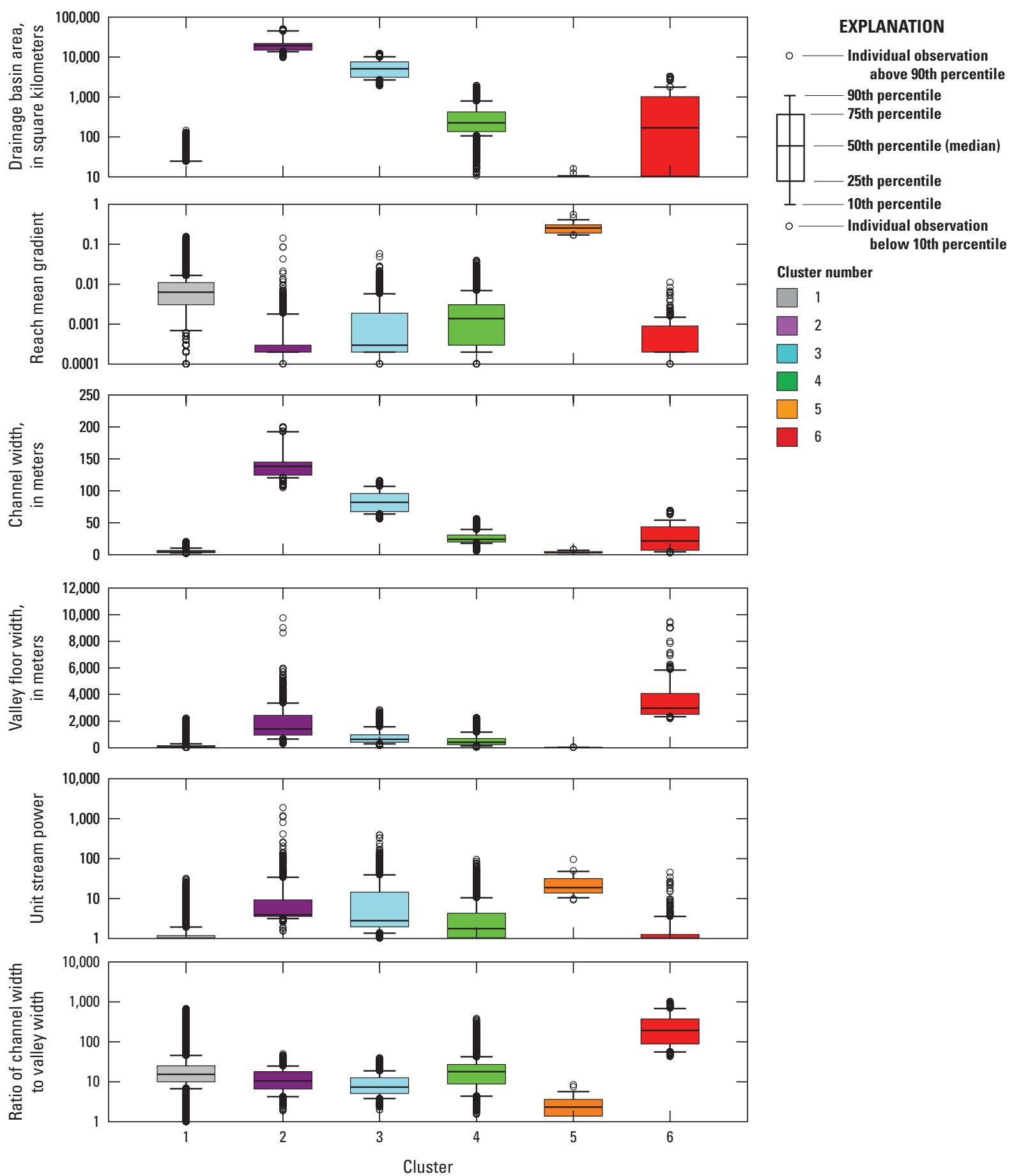

Figure 35. Distributions by cluster of six geomorphic characteristics of stream reaches within the ApalachicolaChattahoochee-Flint River Basin for the 6-cluster classification. 

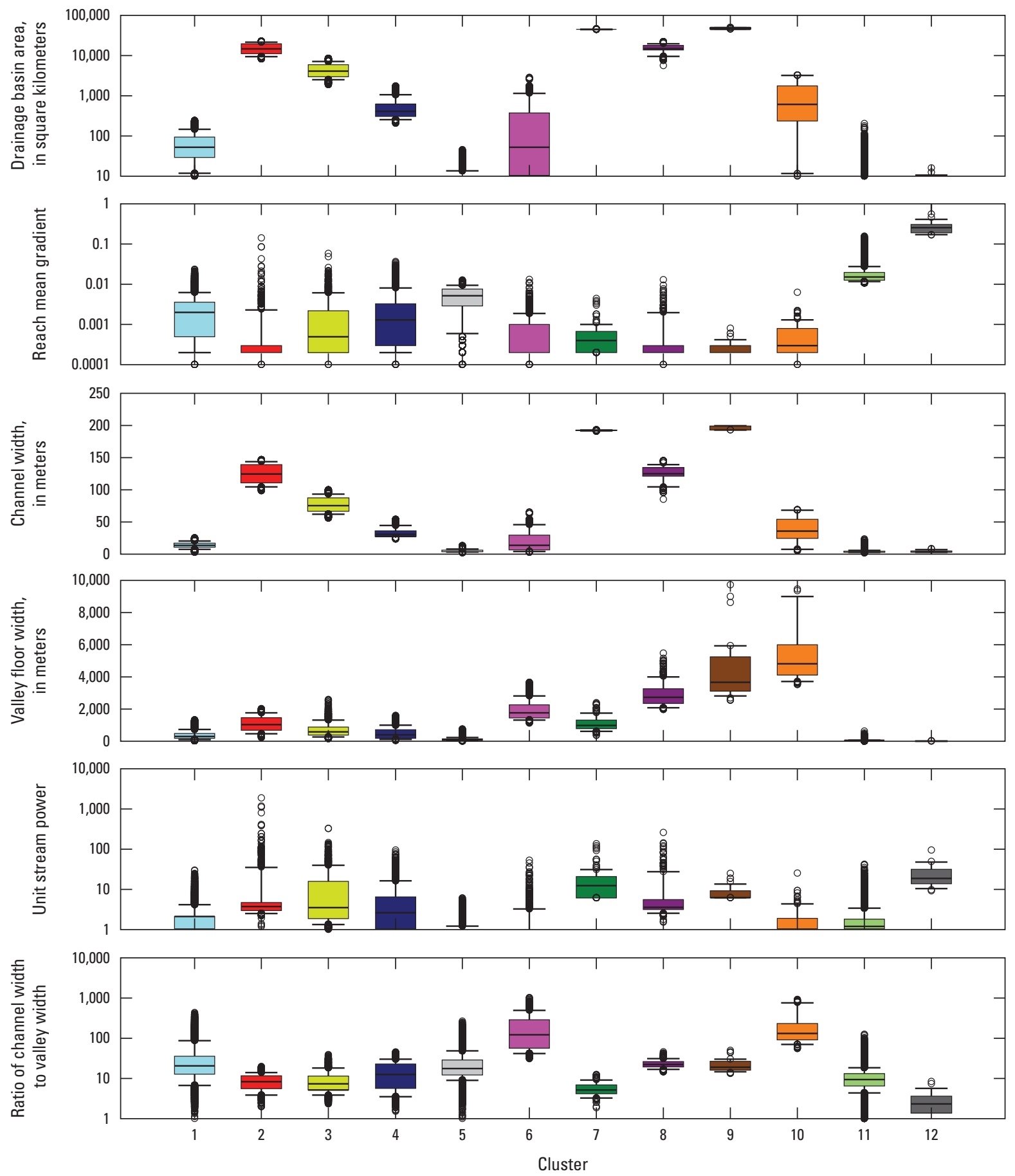

EXPLANATION

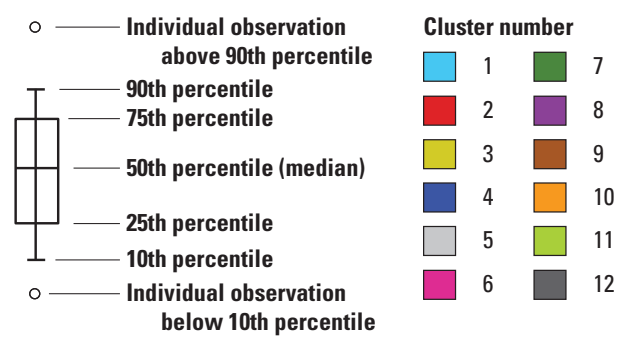

Figure 36. Distributions by cluster of six geomorphic characteristics of stream reaches within the ApalachicolaChattahoochee-Flint River Basin for the 12-cluster classification. 

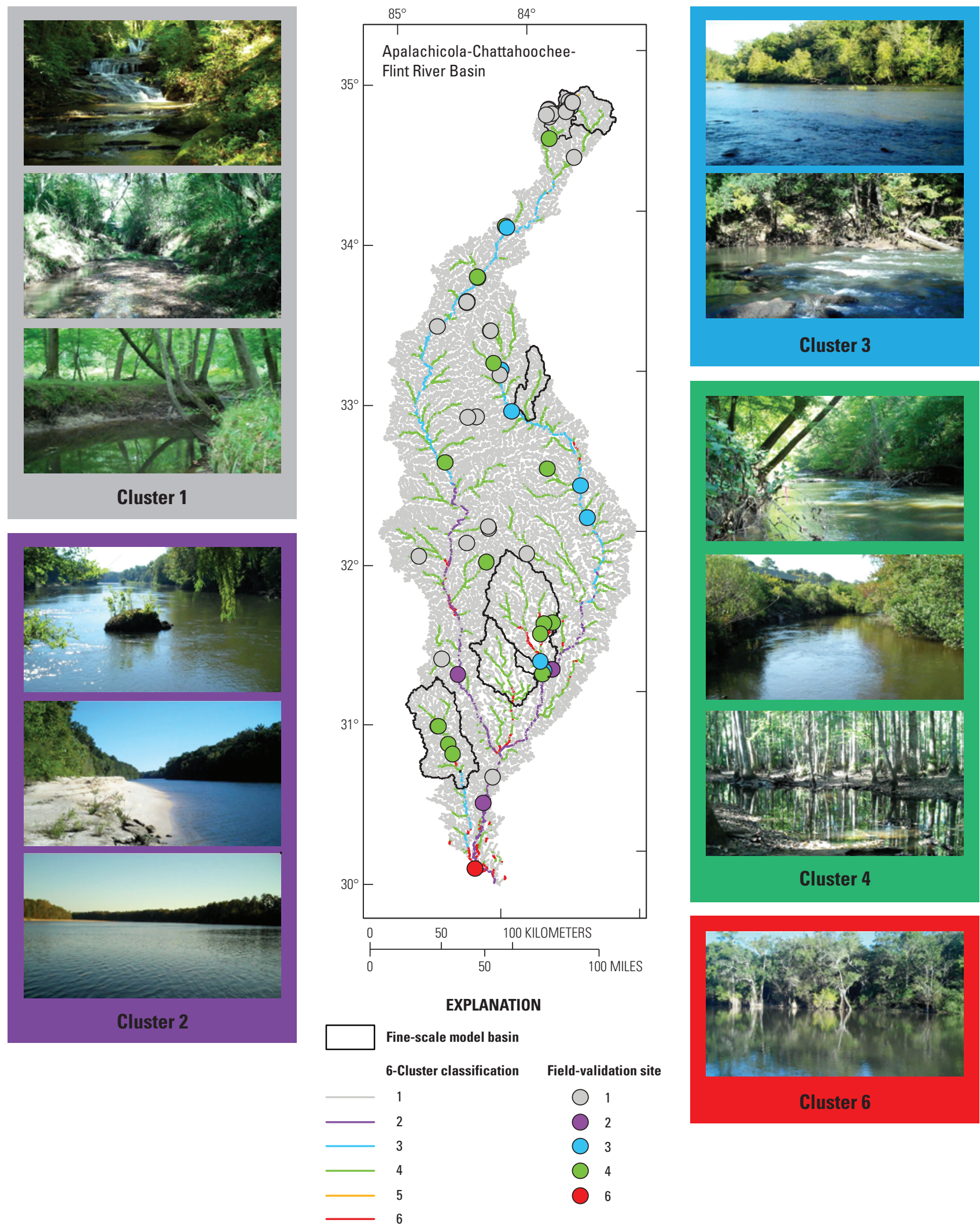

Figure 37. Field-site locations within the Apalachicola-Chattahoochee-Flint River Basin, example photographs, and the 6 -cluster classification. 

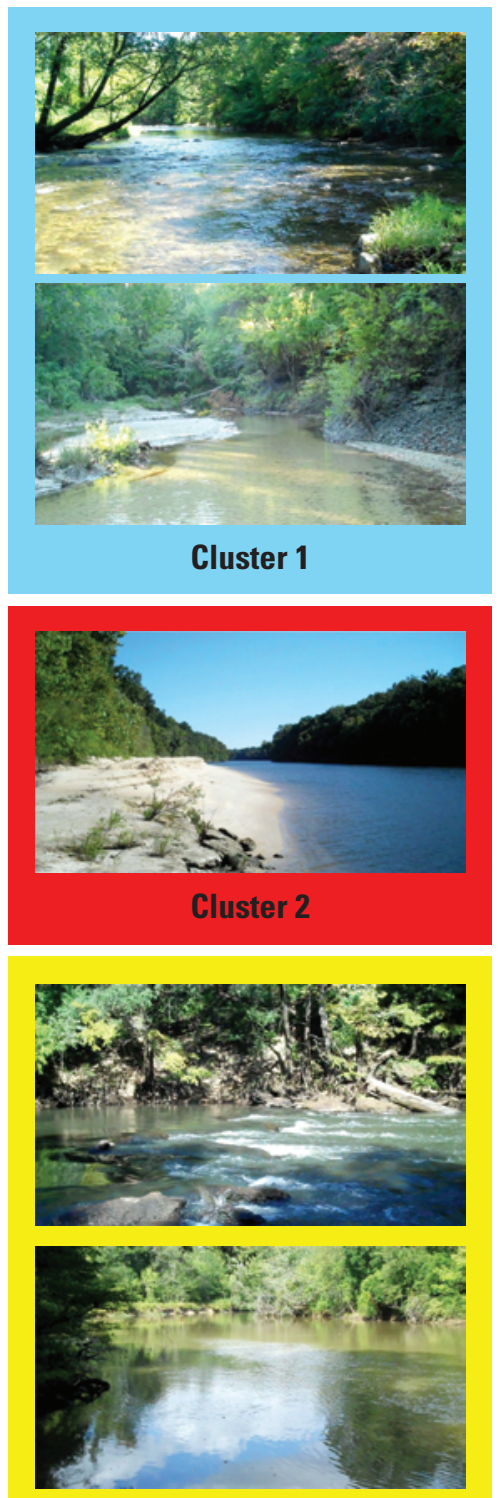

\section{Cluster 3}

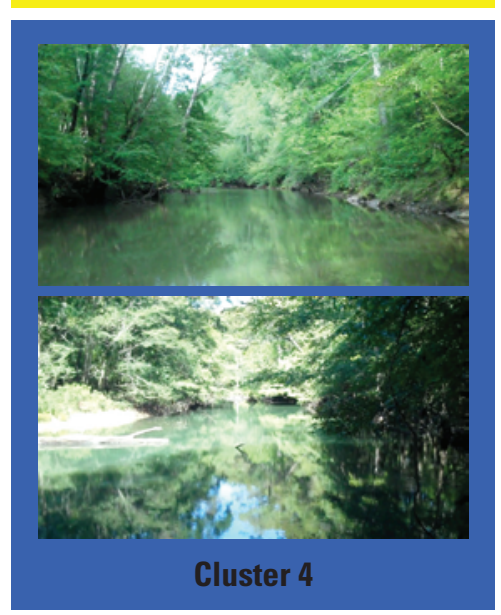

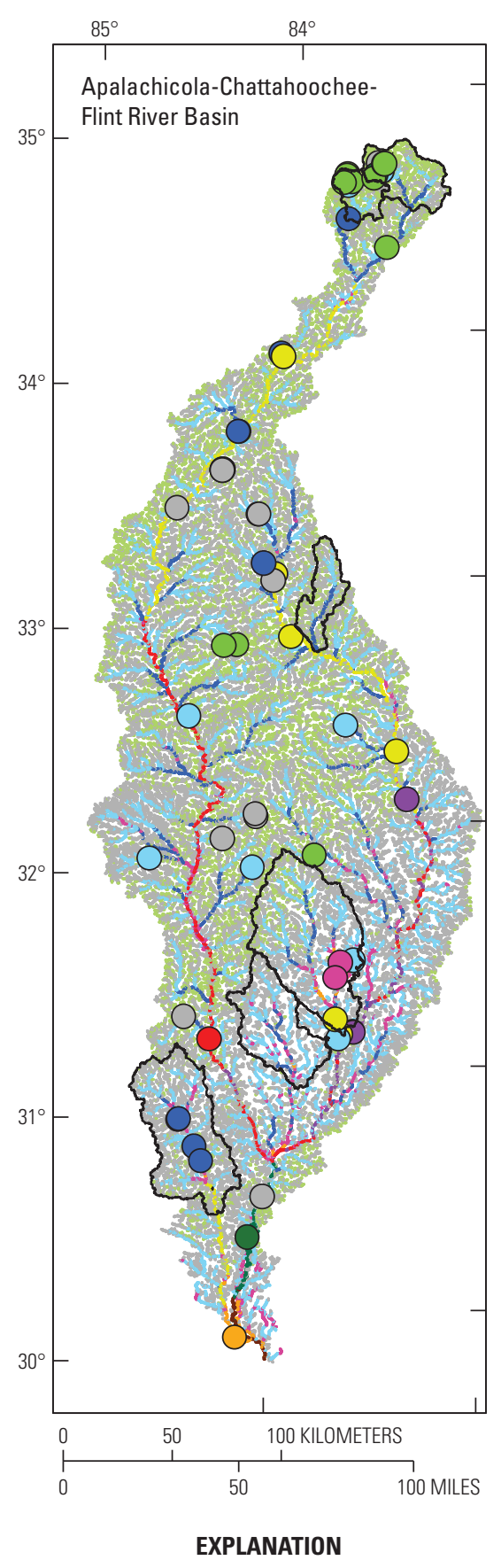

Fine-scale model basin

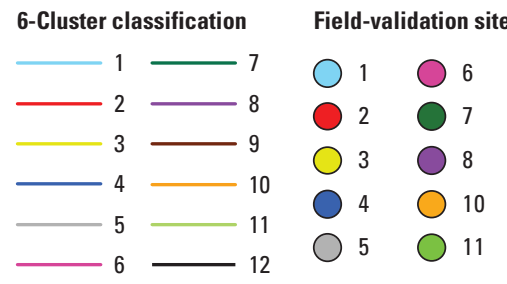

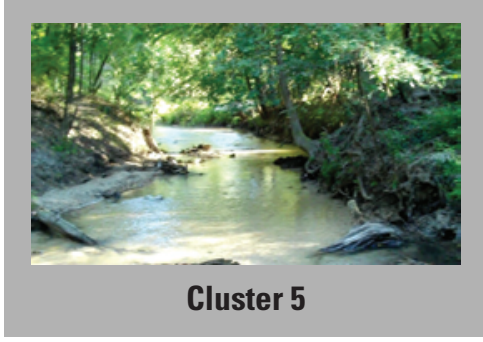
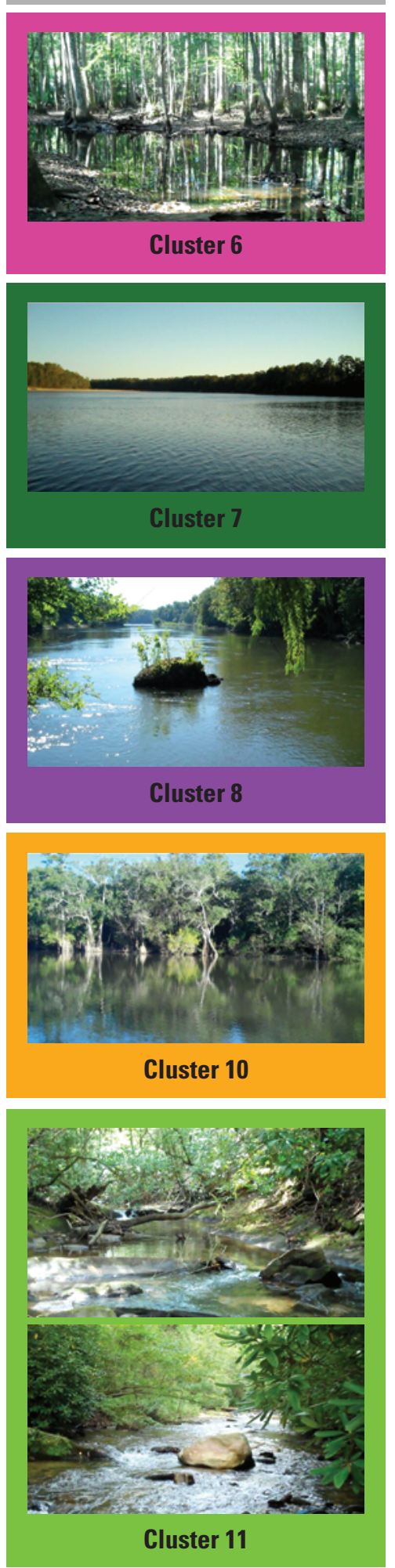

Figure 38. Field-site locations within the Apalachicola-ChattahoocheeFlint River Basin, example photographs, and the 12-cluster classification. 
Table 6. Statistical summary by cluster for selected geomorphic variables for streams in the Apalachicola-Chattahoochee-Flint River Basin for the 6- and 12-cluster classifications.

[Min, minimum value; Max, maximum value; Avg, average value]

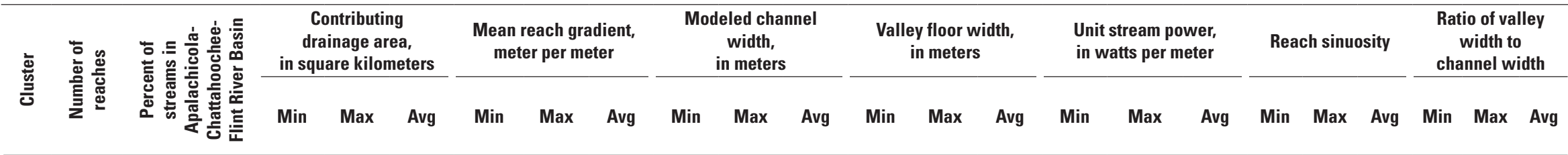

\begin{tabular}{|c|c|c|c|c|c|c|c|c|c|c|c|c|c|c|c|c|c|c|c|c|c|c|c|}
\hline \multicolumn{24}{|c|}{ 6-cluster classification } \\
\hline 1 & 84,945 & 89.89 & 1 & 145 & 10 & 0.0001 & 0.1560 & 0.0083 & 1.7 & 20.5 & 5.8 & 3 & 2,200 & 132 & 0.01 & 31.38 & 1.03 & 1.0 & 4.49 & 1.27 & 1 & 663 & 24 \\
\hline 2 & 969 & 1.03 & 9,557 & 49,735 & 23,020 & 0.0001 & 0.1416 & 0.0011 & 104.9 & 199.5 & 144.2 & 287 & 9,740 & 1,774 & 1.52 & $1,863.44$ & 18.39 & 1.0 & 2.93 & 1.24 & 2 & 49 & 13 \\
\hline 3 & 1,675 & 1.77 & 1,911 & 12,349 & 5,614 & 0.0001 & 0.0578 & 0.0019 & 56.0 & 115.9 & 82.8 & 154 & 2,836 & 780 & 0.54 & 386.62 & 14.09 & 1.0 & 3.28 & 1.23 & 2 & 39 & 10 \\
\hline 4 & 6,461 & 6.84 & 6 & 1,912 & 349 & 0.0001 & 0.0396 & 0.0027 & 5.7 & 56.0 & 26.7 & 39 & 2,243 & 542 & 0.03 & 94.30 & 4.12 & 1.0 & 3.11 & 1.30 & 2 & 378 & 23 \\
\hline 5 & 29 & 0.03 & 1 & 16 & 3 & 0.1675 & 0.5566 & 0.2669 & 3.0 & 8.6 & 4.3 & 3 & 26 & 12 & 9.06 & 94.44 & 24.81 & 1.1 & 1.85 & 1.25 & 1 & 8 & 3 \\
\hline 6 & 419 & 0.44 & 1 & 3,251 & 644 & 0.0001 & 0.0111 & 0.0007 & 3.0 & 68.9 & 26.3 & 2,191 & 9,467 & 3,601 & 0.01 & 44.50 & 1.58 & 1.0 & 2.57 & 1.30 & 42 & 1,015 & 273 \\
\hline \multicolumn{24}{|c|}{ 12-cluster classification } \\
\hline 1 & 12,637 & 13.37 & 1 & 243 & 68 & 0.0001 & 0.0234 & 0.0027 & 2.9 & 25.0 & 14.0 & 16 & 1,329 & 367 & 0.01 & 29.88 & 1.71 & 1.0 & 3.78 & 1.29 & 1 & 429 & 37 \\
\hline 2 & 807 & 0.85 & 8,188 & 22,621 & 15,449 & 0.0001 & 0.1416 & 0.0013 & 98.7 & 146.8 & 125.1 & 217 & 2,022 & 1,078 & 1.18 & $1,863.44$ & 19.87 & 1.0 & 1.85 & 1.15 & 2 & 20 & 9 \\
\hline 3 & 1,361 & 1.44 & 1,902 & 8,439 & 4,524 & 0.0001 & 0.0578 & 0.0021 & 55.9 & 99.9 & 76.9 & 154 & 2,584 & 702 & 0.54 & 326.96 & 14.10 & 1.0 & 3.28 & 1.24 & 2 & 39 & 9 \\
\hline 4 & 2,952 & 3.12 & 205 & 1,728 & 539 & 0.0001 & 0.0364 & 0.0030 & 23.4 & 53.8 & 33.0 & 39 & 1,595 & 484 & 0.14 & 94.30 & 5.93 & 1.0 & 2.53 & 1.33 & 2 & 45 & 15 \\
\hline 5 & 53,597 & 56.72 & 1 & 45 & 6 & 0.0001 & 0.0126 & 0.0052 & 1.8 & 13.0 & 5.2 & 6 & 760 & 121 & 0.01 & 6.00 & 0.65 & 1.0 & 4.49 & 1.27 & 1 & 262 & 25 \\
\hline 6 & 1,066 & 1.13 & 1 & 2,851 & 320 & 0.0001 & 0.0131 & 0.0008 & 2.9 & 65.4 & 19.9 & 1,129 & 3,657 & 1,932 & 0.01 & 52.49 & 1.33 & 1.0 & 2.57 & 1.25 & 31 & 1,015 & 198 \\
\hline 7 & 140 & 0.15 & 44,545 & 45,780 & 45,267 & 0.0002 & 0.0044 & 0.0006 & 191.2 & 193.2 & 192.4 & 354 & 2,382 & 1,097 & 6.09 & 134.93 & 18.03 & 1.0 & 2.93 & 1.60 & 2 & 12 & 6 \\
\hline 8 & 293 & 0.31 & 5,604 & 21,933 & 15,493 & 0.0001 & 0.0129 & 0.0007 & 85.2 & 145.0 & 125.5 & 1,962 & 5,472 & 2,893 & 1.52 & 256.97 & 11.65 & 1.0 & 2.76 & 1.23 & 14 & 45 & 23 \\
\hline 9 & 47 & 0.05 & 45,699 & 49,735 & 47,120 & 0.0002 & 0.0008 & 0.0003 & 193.1 & 199.5 & 195.4 & 2,537 & 9,740 & 4,245 & 6.19 & 24.76 & 8.15 & 1.1 & 2.01 & 1.48 & 13 & 49 & 22 \\
\hline 10 & 131 & 0.14 & 4 & 3,251 & 1,187 & 0.0001 & 0.0063 & 0.0006 & 5.2 & 68.9 & 39.2 & 3,521 & 9,467 & 5,520 & 0.02 & 25.32 & 1.79 & 1.0 & 2.57 & 1.37 & 55 & 908 & 224 \\
\hline 11 & 21,438 & 22.69 & 1 & 202 & 4 & 0.0107 & 0.1560 & 0.0186 & 1.7 & 23.3 & 4.3 & 3 & 613 & 43 & 0.55 & 41.70 & 1.85 & 1.0 & 4.49 & 1.25 & 1 & 123 & 11 \\
\hline 12 & 29 & 0.03 & 1 & 16 & 3 & 0.1675 & 0.5566 & 0.2669 & 3.0 & 8.6 & 4.3 & 3 & 26 & 12 & 9.06 & 94.44 & 24.81 & 1.1 & 1.85 & 1.25 & 1 & 8 & 3 \\
\hline
\end{tabular}




\begin{tabular}{|c|c|c|c|c|c|c|c|}
\hline $\begin{array}{l}\text { Classifica- } \\
\text { tion }\end{array}$ & Cluster & $\begin{array}{l}\text { Dominant } \\
\text { 6-cluster } \\
\text { class }\end{array}$ & $\begin{array}{l}\text { Channel } \\
\text { width }\end{array}$ & Valley width & $\begin{array}{l}\text { Stream- } \\
\text { channel } \\
\text { gradient }\end{array}$ & $\begin{array}{l}\text { Dominant } \\
\text { region }\end{array}$ & Notes \\
\hline \multirow{6}{*}{$\begin{array}{l}\text { 6-cluster } \\
\text { class }\end{array}$} & 1 & -- & Low & Low & Low-medium & Entire basin & Small streams throughout the Apalachicola-Chattahoochee-Flint River Basin, $90 \%$ classified as confined. \\
\hline & 2 & -- & High & Low-medium & Very low & $\begin{array}{l}\text { Piedmont and } \\
\text { Coastal Plain }\end{array}$ & $\begin{array}{l}\text { Large rivers including the lower Flint, lower Chattahoochee, and lower Apalachicola Rivers, } 60 \% \text { of } \\
\text { reaches classified as lakes or reservoirs (by the NWI). }\end{array}$ \\
\hline & 3 & -- & Medium & Low-medium & Very low & $\begin{array}{l}\text { Piedmont and } \\
\text { Coastal Plain }\end{array}$ & $\begin{array}{l}\text { Medium-sized main-stem river reaches of the middle Flint and Chattahoochee Rivers and the Chipola } \\
\text { River. Generally higher gradient than class } 2 \text { streams, } 25 \% \text { of reaches classified as lakes/reservoirs (by } \\
\text { the NWI). }\end{array}$ \\
\hline & 4 & -- & Low-medium & Low-medium & Very low & Entire basin & $\begin{array}{l}\text { Low gradient medium-sized streams, } 50 \% \text { classified as freshwater forested or shrub wetlands and } 20 \% \\
\text { as riverine wetlands (by the NWI). }\end{array}$ \\
\hline & 5 & -- & Very low & Very low & Very high & Blue Ridge & Very high gradient streams and waterfalls. Small streams in steep terrain and confined in narrow valleys. \\
\hline & 6 & -- & Medium & Very wide & Very low & Coastal Plain & $\begin{array}{l}\text { Reaches with very high valley widths. Includes reservoir reaches }(20 \%) \text { of the Chattahoochee and Flint } \\
\text { Rivers and wide reaches of Ichawaynochaway Creek and other streams in the Coastal Plain. High } \\
\text { percentage of reaches freshwater forested/shrub wetlands }(40 \%) \text { or freshwater emergent wetlands } \\
(40 \%) \text { (by the NWI). }\end{array}$ \\
\hline \multirow[t]{12}{*}{$\begin{array}{l}\text { 12-cluster } \\
\text { class }\end{array}$} & 1 & 1,4 & Low & Low & Low & Entire basin & $\begin{array}{l}\text { Medium-small streams throughout the Apalachicola-Chattahoochee-Flint River Basin, } 60 \% \text { of flood- } \\
\text { plains classified as freshwater forested/shrub wetlands. }\end{array}$ \\
\hline & 2 & 2,3 & High & Low & Low & Entire basin & $\begin{array}{l}\text { Main-stem reaches of the middle Flint and Chattahoochee Rivers. Narrower valleys than class } 8,80 \% \text { of } \\
\text { reaches classified as lake/reservoir (by the NWI). }\end{array}$ \\
\hline & 3 & 3 & High & Low-medium & Low & $\begin{array}{l}\text { Piedmont and } \\
\text { Coastal Plain }\end{array}$ & $\begin{array}{l}\text { Reaches of the Chattahoochee River downstream from Lake Lanier, the middle Flint River, and the } \\
\text { Chipola River. Generally higher gradients than class 2, } 75 \% \text { of floodplains classified by the NWI as } \\
\text { riverine wetland. }\end{array}$ \\
\hline & 4 & 4 & Medium & Low & Low & $\begin{array}{l}\text { Piedmont and } \\
\text { Coastal Plain }\end{array}$ & $\begin{array}{l}\text { Medium-sized low gradient streams throughout the Piedmont and Coastal Plain. One half of all reaches } \\
\text { associated with freshwater forested/shrub wetlands (by the NWI). }\end{array}$ \\
\hline & 5 & 1 & Very low & Very low & Very low & Coastal Plain & $\begin{array}{l}\text { Small streams throughout the Apalachicola-Chattahoochee-Flint River basin. High percentage of streams } \\
\text { confined, } 50 \% \text { freshwater forested/shrub wetlands and } 40 \% \text { not classified as wetland by the NWI. }\end{array}$ \\
\hline & 6 & 4,6 & Low-medium & Low-medium & Very low & $\begin{array}{l}\text { Piedmont and } \\
\text { Coastal Plain }\end{array}$ & $\begin{array}{l}\text { Variable drainage basin area, intermediate gradient, wide. Nearly } 40 \% \text { of reaches associated with lakes } \\
\text { and reservoirs, and } 45 \% \text { associated with freshwater forested/shrub wetlands (by the NWI). }\end{array}$ \\
\hline & 7 & 2 & High & Low-medium & Very low & Coastal Plain & $\begin{array}{l}\text { Apalachicola River only. Narrower valleys than cluster } 9 \text { reaches. Nearly all reaches associated with } \\
\text { riverine wetlands (by the NWI). }\end{array}$ \\
\hline & 8 & 2,3 & Medium-high & Medium-high & Very low & Coastal Plain & $\begin{array}{l}\text { Similar to cluster 2; middle and lower Flint and middle Chattahoochee River reaches. Wider valleys than } \\
\text { cluster } 2 \text {. }\end{array}$ \\
\hline & 9 & 2 & Very high & Medium-high & Very low & Coastal Plain & $\begin{array}{l}\text { Lower reaches of Apalachicola River only. Wider valleys than class } 7 \text { reaches. All reaches associated } \\
\text { with riverine wetlands (by the NWI). }\end{array}$ \\
\hline & 10 & 6 & Low-medium & Very high & Very low & Coastal Plain & $\begin{array}{l}\text { Medium-sized streams in very wide valleys. High valley width to channel width ratio, } 45 \% \text { riverine and } \\
40 \% \text { freshwater forested/shrub wetlands (by the NWI). }\end{array}$ \\
\hline & 11 & 1 & Low & Low & Low-medium & Entire basin & $\begin{array}{l}\text { Small streams throughout the basin, although less common in the Coastal Plain and generally associ- } \\
\text { ated with steeper terrain. Higher gradient than class } 5 \text {. Low association with wetlands }(<30 \% \text {, by the } \\
\text { NWI). }\end{array}$ \\
\hline & 12 & 5 & Very low & Very low & Very high & Blue Ridge & Very high gradient streams and waterfalls. Small streams in steep terrain and confined in narrow valleys. \\
\hline
\end{tabular}


Table 8. Comparative support for logistic regression models for the Halloween darter and bluestripe shiner occurrence based on geomorphology variables and classes from a 6-cluster classification. Models with Akaike Information Criterion (AIC) values within 15 of the best-supported model, along with the null model for comparison.

[AIC, Akaike Information Criterion; D AIC, AIC deviance from the AIC value for the best-supported model; area ${ }^{2}$, area squared]

\begin{tabular}{lccc}
\hline \multicolumn{1}{c}{ Model variables } & $\begin{array}{c}\text { Number of } \\
\text { parameters }\end{array}$ & AIC & D AIC \\
\hline A. Using geomorphology variables & Halloween darter & & \\
Area, gradient, ratio, sinuosity & & & \\
Area, gradient, ratio & 4 & 326.2 & 1.3 \\
Area, gradient, sinuosity & 3 & 327.5 & 7.7 \\
Area, ratio & 3 & 333.9 & 9 \\
Area, sinuosity & 2 & 335.2 & 12.9 \\
B. Using stream classification & 2 & 339.1 & 0 \\
Cluster 3, Cluster 4 & & & ${ }^{1} 1.8$ \\
Cluster 1, Cluster 3, Cluster 4 & 2 & 208.8 & ${ }^{12.5}$ \\
Cluster 1, Cluster 2, Cluster 3 & 3 & 210.6 & ${ }^{3} 3.8$ \\
Cluster 1, Cluster 2, Cluster 3, Cluster 4 & 3 & 211.3 & 14.5 \\
Cluster 3 & 4 & 212.6 & $42.2(\mathrm{~A})$ \\
Null model (intercept only) & 1 & 223.3 & 159.6 (B) \\
\hline
\end{tabular}

\begin{tabular}{llll}
\hline A. Using geomorphology variables & & & \\
Area, area $^{2}$, ratio, sinuosity & 4 & 265.05 & 0 \\
Area, rrea $^{2}$, ratio & 3 & 266.21 & 1.16 \\
Area, rrea $^{2}$, ratio, sinuosity, gradient & 5 & 266.84 & ${ }^{1} 1.79$ \\
Area, area & , ratio, gradient & 267.9 & 2.85 \\
B. Using stream classification & 4 & & \\
Cluster 2, Cluster 3, Cluster 4 & & 278.07 & 0 \\
Cluster 1, Cluster 2, Cluster 3 & 3 & 279.25 & 1.18 \\
Cluster 1, Cluster 2, Cluster 3, Cluster 4 (or 2, 3, 4, and 6) & 3 & 279.51 & ${ }^{1} 1.44$ \\
Cluster 1, Cluster 3, Cluster 4 & 4 & 281.41 & 3.34 \\
Cluster 3, Cluster 4 & 3 & 283.83 & 5.76 \\
Cluster 2, Cluster 3 & 2 & 287.9 & 9.83 \\
Null model (intercept only) & 2 & 371.68 & 106.6 (A) \\
& 1 & & 93.6 (B) \\
\hline
\end{tabular}

${ }^{1}$ Although within about $2-4$ of the lowest AIC, adding cluster variables did not indicate support for the more complex models because the AIC deviance mostly was the penalty for additional terms with little increase in model likelihood. 
Table 9. Regression coefficient estimates for best-supported logistic regression models of the occurrence of the Halloween darter and bluestripe shiner based on geomorphology variables and on a 6-cluster classification for the Apalachicola-Chattahoochee-Flint River Basin.

[SE, standard error; area ${ }^{2}$, area squared]

\begin{tabular}{|c|c|}
\hline Variable & Estimate (SE) \\
\hline \multicolumn{2}{|c|}{ Halloween darter } \\
\hline \multicolumn{2}{|c|}{${ }^{1}$ Best-supported geomorphic variable model } \\
\hline Intercept & $-2.9583(0.2028)$ \\
\hline Area & $0.6319(0.1777)$ \\
\hline Gradient & $-0.6701(0.2476)$ \\
\hline Ratio & $-1.0999(0.3424)$ \\
\hline Sinuosity & $0.2073(0.1092)$ \\
\hline \multicolumn{2}{|c|}{ Best-supported classification model } \\
\hline Intercept & $-4.2826(0.3806)$ \\
\hline Cluster 3 & $5.6371(0.5496)$ \\
\hline Cluster 4 & $1.8988(0.4784)$ \\
\hline \multicolumn{2}{|c|}{ Bluestripe shiner } \\
\hline \multicolumn{2}{|c|}{${ }^{1}$ Best-supported geomorphic variable model } \\
\hline Intercept & $-1.931(0.2151)$ \\
\hline Area & $2.6221(0.3966)$ \\
\hline Area $^{2}$ & $-2.1157(0.4029)$ \\
\hline Ratio & $-1.6369(0.3566)$ \\
\hline Sinuosity & $0.2696(0.1487)$ \\
\hline \multicolumn{2}{|c|}{ Best-supported classification model } \\
\hline Intercept & $-2.6331(0.2989)$ \\
\hline Cluster 2 & $2.6331(0.8695)$ \\
\hline Cluster 3 & $3.9140(0.4659)$ \\
\hline Cluster 4 & $1.2368(0.3731)$ \\
\hline
\end{tabular}

${ }^{1}$ Geomorphic variables are each standardized by subtracting the mean and dividing by the standard deviation. 
Table 10. Prediction error rates for best-supported models of occurrence of Halloween darter and bluestripe shiner, based on either individual geomorphic variables or stream classification.

\begin{tabular}{|c|c|c|c|}
\hline Model & $\begin{array}{c}\text { Number of } \\
\text { predicted presences }\end{array}$ & Commission error & Omission error \\
\hline \multicolumn{4}{|c|}{ Halloween darter } \\
\hline Geomorphic variables & 5 & 0.8 & 0.98 \\
\hline Classification & 39 & 0.2 & 0.39 \\
\hline \multicolumn{4}{|c|}{ Bluestripe shiner } \\
\hline Geomorphic variables & 42 & 0.19 & 0.55 \\
\hline Classification & 49 & 0.27 & 0.53 \\
\hline
\end{tabular}

Table 11. Total number of segments in each cluster of the 6-cluster classification scheme, number of segments with known Halloween darter and Bluestripe shiner occurrence, and mean predicted probability of occurrence based on the best-supported geomorphic and classification models using all observations.

\begin{tabular}{|c|c|c|c|c|}
\hline \multirow{2}{*}{${ }^{1}$ Cluster } & \multirow{2}{*}{$\begin{array}{l}\text { Number of } \\
\text { segments }\end{array}$} & \multirow{2}{*}{$\begin{array}{c}\text { Number of segments } \\
\text { with target species } \\
\text { occurrence }\end{array}$} & \multicolumn{2}{|c|}{$\begin{array}{l}\text { Mean predicted probability of } \\
\text { target species occurrence }\end{array}$} \\
\hline & & & $\begin{array}{l}\text { Geomorphic variable } \\
\text { model }\end{array}$ & Classification model \\
\hline \multicolumn{5}{|c|}{ Halloween darter } \\
\hline 1 & 507 & 7 & 0.056 & 0.014 \\
\hline 2 & 3 & 0 & 0.916 & 0.014 \\
\hline 3 & 39 & 31 & 0.251 & 0.795 \\
\hline 4 & 154 & 13 & 0.066 & 0.084 \\
\hline 6 & 4 & 0 & 0.002 & 0.014 \\
\hline \multicolumn{5}{|c|}{ Bluestripe shiner } \\
\hline 1 & 175 & 12 & 0.128 & 0.075 \\
\hline 2 & 6 & 3 & 0.422 & 0.5 \\
\hline 3 & 46 & 36 & 0.731 & 0.78 \\
\hline 4 & 126 & 25 & 0.138 & 0.198 \\
\hline 6 & 4 & 0 & 0.002 & 0.075 \\
\hline
\end{tabular}

${ }^{1}$ None of the segments in the darter or minnow datasets were assigned to cluster 5 . 
Table 12. Species distributions from collection records by cluster for the 6- and 12-cluster classifications of streams within the Apalachicola-Chattahoochee-Flint River Basin for two darters and two minnows.

\begin{tabular}{|c|c|c|c|c|c|c|c|c|c|c|c|}
\hline $\begin{array}{c}\text { 6-cluster } \\
\text { classification }\end{array}$ & $\begin{array}{c}\text { Percent } \\
\text { Apalachicola- } \\
\text { Chattahoochee- } \\
\text { Flint River Basin }\end{array}$ & $\begin{array}{l}\text { Count } \\
\text { blackbanded } \\
\text { darter only }\end{array}$ & $\begin{array}{c}\text { Count } \\
\text { blackbanded } \\
\text { darter all }\end{array}$ & $\begin{array}{c}\text { Count } \\
\text { Halloween } \\
\text { darter all }\end{array}$ & $\begin{array}{c}\text { Percent } \\
\text { blackbanded } \\
\text { darter only }\end{array}$ & $\begin{array}{c}\text { Percent } \\
\text { blackbanded } \\
\text { darter all }\end{array}$ & $\begin{array}{c}\text { Percent } \\
\text { Halloween } \\
\text { darter all }\end{array}$ & $\begin{array}{c}\text { Count } \\
\text { blacktail } \\
\text { shiner only }\end{array}$ & $\begin{array}{c}\text { Count } \\
\text { bluestripe } \\
\text { shiner all }\end{array}$ & $\begin{array}{c}\text { Percent } \\
\text { blacktail } \\
\text { shiner only }\end{array}$ & $\begin{array}{l}\text { Percent } \\
\text { bluestripe } \\
\text { shiner all }\end{array}$ \\
\hline 1 & 76.2 & 500 & 507 & 7 & 89.9 & 71.7 & 13.7 & 162 & 13 & 57.9 & 16.9 \\
\hline 2 & 0.5 & 3 & 3 & 0 & 1.0 & 0.4 & 0.0 & 3 & 3 & 1.1 & 3.9 \\
\hline 3 & 1.2 & 8 & 39 & 31 & 1.8 & 5.5 & 60.8 & 10 & 36 & 3.6 & 46.8 \\
\hline 4 & 21.5 & 141 & 154 & 13 & 6.8 & 21.8 & 25.5 & 101 & 25 & 36.1 & 32.5 \\
\hline 5 & 0.0 & 0 & 0 & 0 & 0.0 & 0.0 & 0.0 & 0 & 0 & 0.0 & 0.0 \\
\hline 6 & 0.6 & 4 & 4 & 0 & 0.4 & 0.6 & 0.0 & 4 & 0 & 1.4 & 0.0 \\
\hline Total & & 656 & 707 & 51 & & & & 280 & 77 & & \\
\hline $\begin{array}{c}\text { 12-cluster } \\
\text { classification }\end{array}$ & $\begin{array}{c}\text { Percent } \\
\text { Apalachicola- } \\
\text { Chattahoochee- } \\
\text { Flint River Basin }\end{array}$ & $\begin{array}{c}\text { Count } \\
\text { blackbanded } \\
\text { darter only }\end{array}$ & $\begin{array}{c}\text { Count } \\
\text { blackbanded } \\
\text { darter all }\end{array}$ & $\begin{array}{c}\text { Count } \\
\text { Halloween } \\
\text { darter all }\end{array}$ & $\begin{array}{c}\text { Percent } \\
\text { blackbanded } \\
\text { darter only }\end{array}$ & $\begin{array}{c}\text { Percent } \\
\text { blackbanded } \\
\text { darter all }\end{array}$ & $\begin{array}{c}\text { Percent } \\
\text { Halloween } \\
\text { darter all }\end{array}$ & $\begin{array}{c}\text { Count black- } \\
\text { tail shiner } \\
\text { only }\end{array}$ & $\begin{array}{c}\text { Count } \\
\text { bluestripe } \\
\text { shiner all }\end{array}$ & $\begin{array}{c}\text { Percent } \\
\text { blacktail } \\
\text { shiner only }\end{array}$ & $\begin{array}{c}\text { Percent } \\
\text { bluestripe } \\
\text { shiner all }\end{array}$ \\
\hline 1 & 13.4 & 287 & 293 & 6 & 43.8 & 41.4 & 11.8 & 156 & 15 & 55.7 & 19.5 \\
\hline 2 & 0.9 & 0 & 0 & 0 & 0.0 & 0.0 & 0.0 & 3 & 1 & 1.1 & 1.3 \\
\hline 3 & 1.4 & 8 & 36 & 28 & 1.2 & 5.1 & 54.9 & 7 & 35 & 2.5 & 45.5 \\
\hline 4 & 3.1 & 50 & 59 & 9 & 7.6 & 8.3 & 17.6 & 44 & 19 & 15.7 & 24.7 \\
\hline 5 & 56.7 & 214 & 217 & 3 & 32.6 & 30.7 & 5.9 & 46 & 3 & 16.4 & 3.9 \\
\hline 6 & 1.1 & 7 & 9 & 2 & 1.1 & 1.3 & 3.9 & 7 & 0 & 2.5 & 0.0 \\
\hline 7 & 0.1 & 1 & 1 & 0 & 0.2 & 0.1 & 0.0 & 0 & 0 & 0.0 & 0.0 \\
\hline 8 & 0.3 & 2 & 4 & 2 & 0.3 & 0.6 & 3.9 & 2 & 3 & 0.7 & 3.9 \\
\hline 9 & 0.0 & 0 & 0 & 0 & 0.0 & 0.0 & 0.0 & 0 & 0 & 0.0 & 0.0 \\
\hline 10 & 0.1 & 3 & 3 & 0 & 0.5 & 0.4 & 0.0 & 3 & 0 & 1.1 & 0.0 \\
\hline 11 & 22.7 & 84 & 85 & 1 & 12.8 & 12.0 & 2.0 & 12 & 1 & 4.3 & 1.3 \\
\hline 12 & 0.0 & 0 & 0 & 0 & 0.0 & 0.0 & 0.0 & 0 & 0 & 0.0 & 0.0 \\
\hline Total & & 656 & 707 & 51 & & & & 280 & 77 & & \\
\hline
\end{tabular}




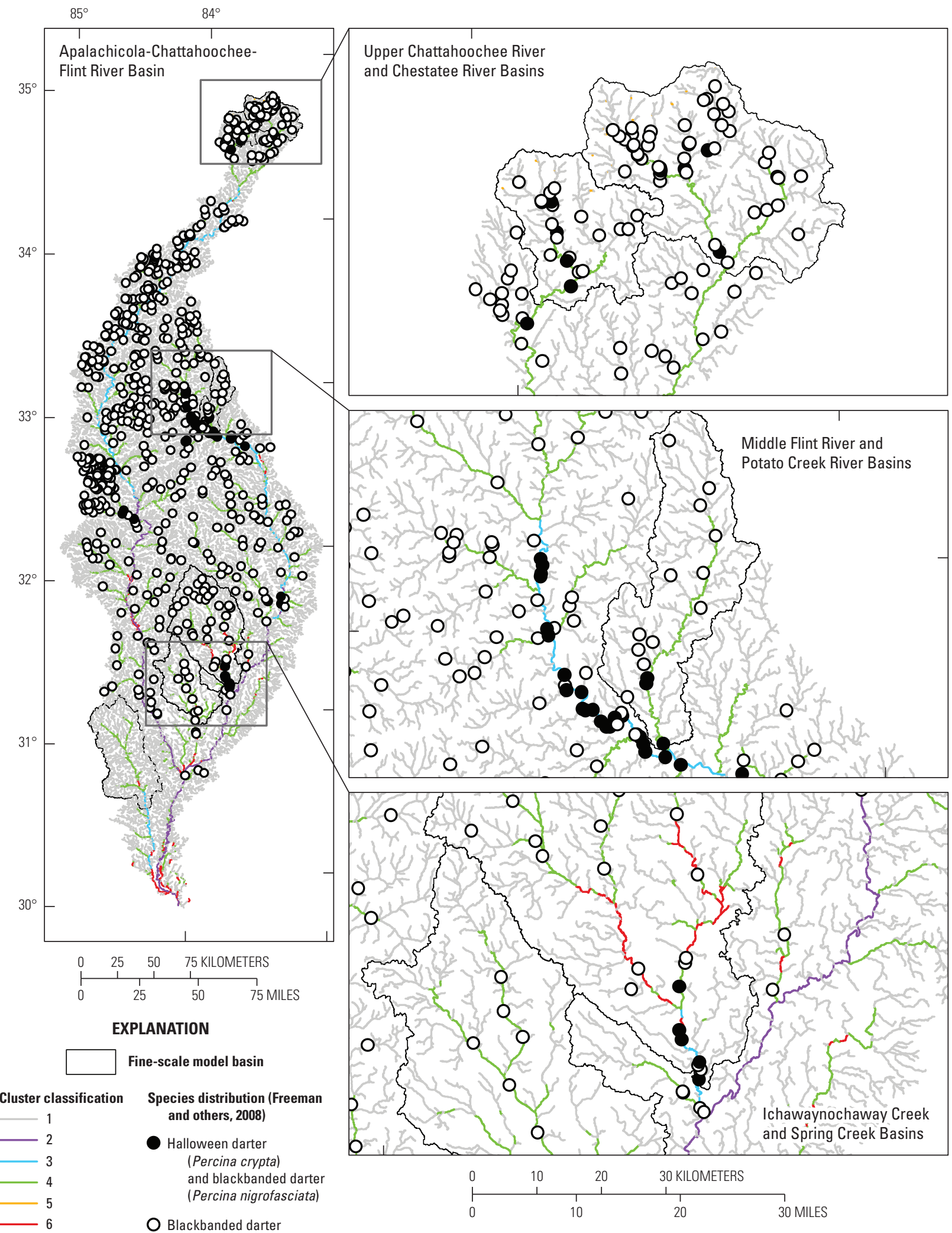

Figure 39. Species distributions of the Halloween darter (Percina crypta) and blackbanded darter (Percina nigrofaciata) and the 6-cluster classification of stream reaches in the Apalachicola-Chattahoochee-Flint River Basin with details shown in three regions. 


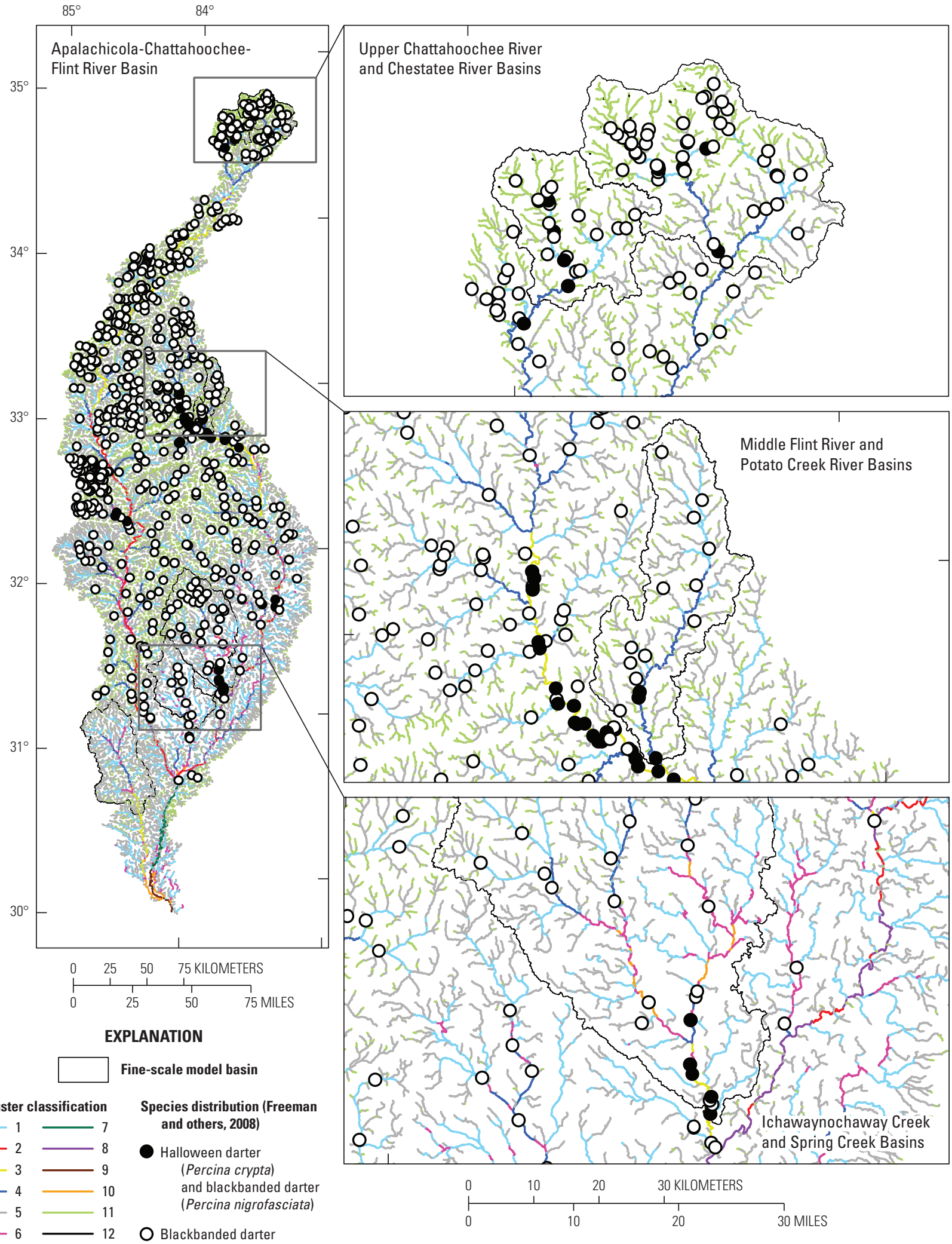

Figure 40. Species distributions of Halloween darter (Percina crypta) and blackbanded darter (Percina nigrofaciata) and the 12-cluster classification of stream reaches in the Apalachicola-Chattahoochee-Flint River Basin with details shown in three regions. 


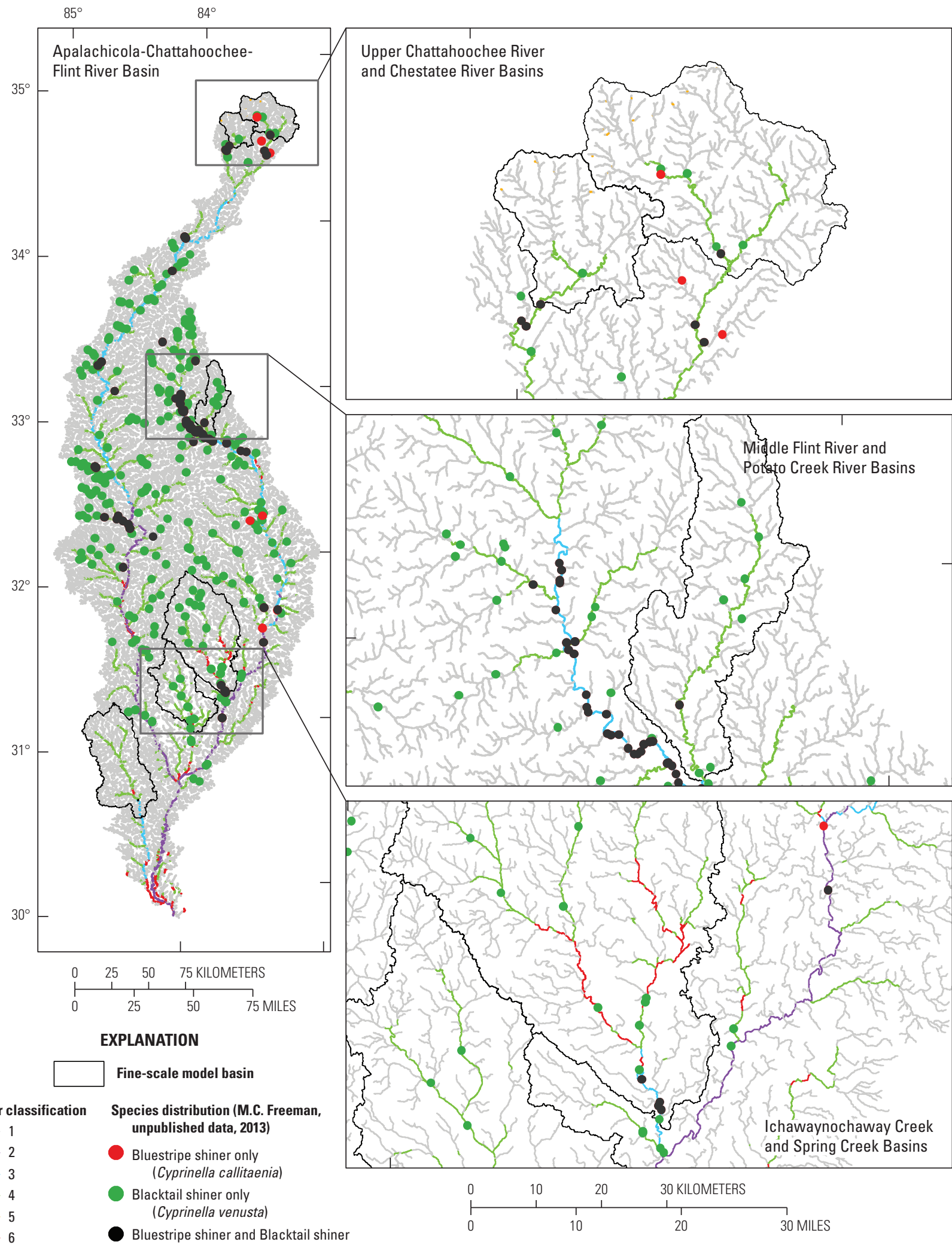

Figure 41. Species distributions of bluestripe shiner (Cyprinella callitaenia) and blacktail shiner (Cyprinella venusta) and the 6-cluster classification of stream reaches in the Apalachicola-Chattahoochee-Flint River Basin with details shown in three regions. 


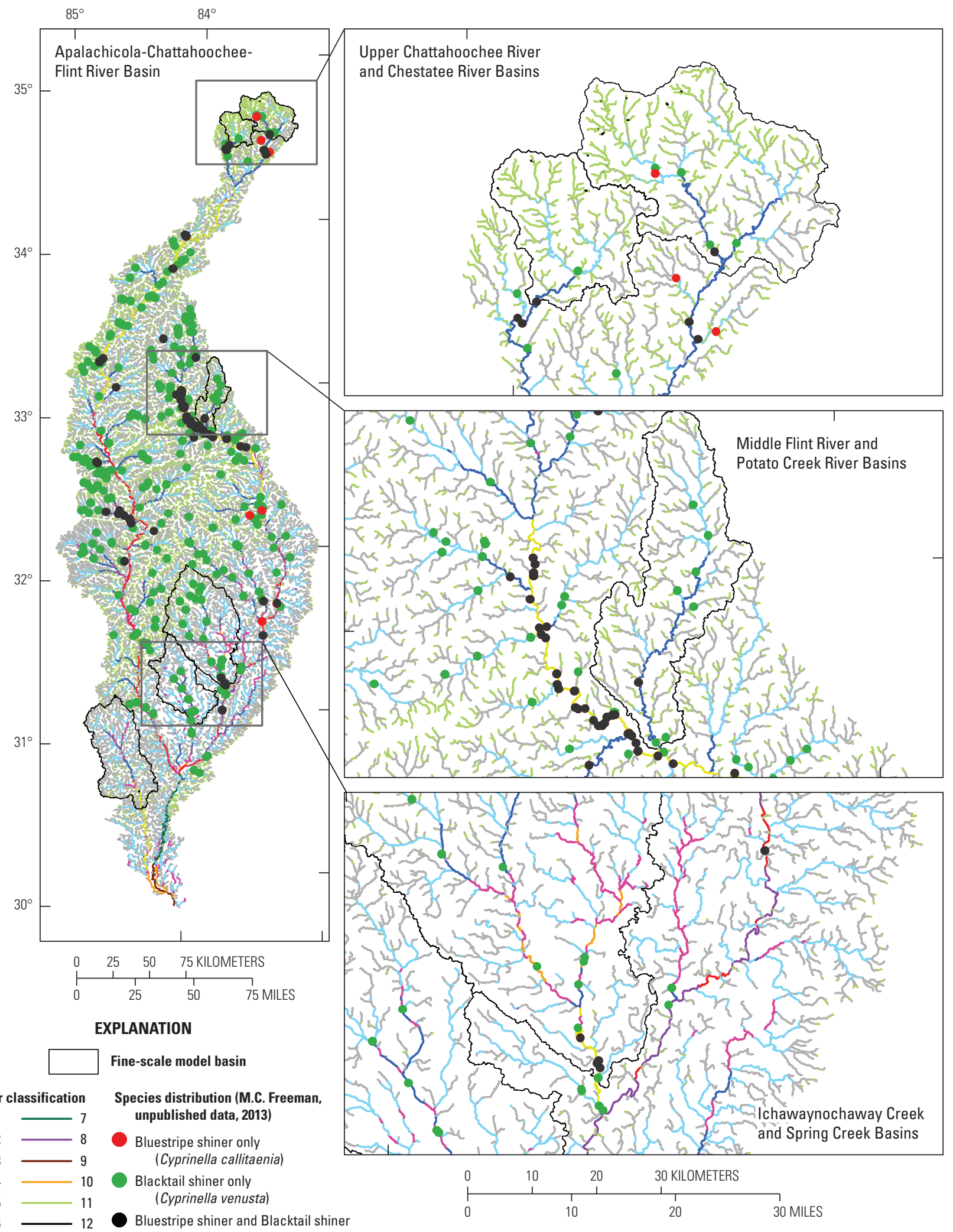

Figure 42. Species distributions of bluestripe shiner (Cyprinella callitaenia) and blacktail shiner (Cyprinella venusta) and the 12-cluster classification of stream reaches in the Apalachicola-Chattahoochee-Flint River Basin with details shown in three regions. 


\section{Discussion}

The stream classification developed for the ApalachicolaChattahoochee-Flint River Basin was produced using the best available basin-wide GIS data during the 2009-2011 time period. There are inherent limitations to the datasets currently available at the landscape scale, which limited our ability to accurately predict some biologically relevant channel features such as flood plain connectivity. Our approach uses landscapescale characteristics to predict the spatial distribution of stream-channel classes at the reach scale, and the biological significance of these classes will vary by application.

\section{Stream Network}

Development of the classification for the ApalachicolaChattahoochee-Flint required a systematically derived stream network. There are limitations in using a 30-m scale DEMderived network for a basin-wide reach-scale stream classification, but in general DEM-derived streams are close to locations mapped from other sources. The DEM-derived stream network deviates substantially from the NHD and aerial photography in the Coastal Plain's Dougherty Plain and Upper Eocene Ocala limestone (fig. 3). This landscape is dominated by limestone dissolution and contains many sinks, depressions, and underground streams (Torak and Painter, 2006). In addition, in the DEM-based network, headwater streams do not always match up with the NHD network because topographic methods for detecting stream initiations are not always accurate. The 30-m resolution of the DEM has inherent limitations in uplands where variable geology, surficial geology, and land-use history may diminish the topographic signature of a stream channel.

An alternative approach would have been to derive the network from the NHD. This approach was avoided in this study for a number of reasons: (1) the higher resolution versions of the NHD would require subjective and labor-intensive pruning to simplify it to meet SERAP needs, (2) NHD content indicated variable drainage network density across the study areas (complicating 1), and (3) although accurate, NHD features were not all connected to the overall network (that is, there were sinks terminating closed basins). The SERAP models required that no streams be disconnected from the overall study area networks in order to be able to route water successfully. The NHD versions available at the time this project began, 2009, also had some inherent limitations, including lack of or variable directionality, inconsistent or inaccurate attributes, and highly variable stream densities in headwater areas and karst regions with intermittent streams. A DEMderived stream network provided the means to define a consistent network throughout the Apalachicola-Chattahoochee-Flint River Basin, as needed for hydrologic modeling, although perhaps at the loss of some detail.
The regional scale of the classification for the Apalachicola-Chattahoochee-Flint River Basin may miss some potentially important stream habitat features because of the DEM resolution. The DEM-derived stream network in structure is represented by a single-thread channel and does not include information such as multithreaded channels, off-channel and flood-plain features such as lakes and reservoirs, karst features such as sinkholes, or indicators of flood-plain connectivity such as adjacent swamps and wetlands. The DEM-derived, basin-wide classification also fails to capture sinuosity at smaller scales, although sinuosity is captured well at some scales, particularly for canyon reaches of smaller streams and for the Apalachicola River, a large channelized river with distinctive banks in a low-gradient flood plain.

\section{Stream Confinement}

Our measure of stream confinement differs substantially from the measure used by Peterson and others (2009) for the lower Flint River, which was determined to be a useful predictor of potential for habitat change and fish abundance, but was based on confinement within the banks of the river (or flood-plain incision) rather than confinement of the stream channel within the valley (Peterson and others, 2009). Confined streams were defined by Peterson and others (2009) as single-threaded with high, well-defined banks (bankfull width to depth ratios less than 10), and infrequent overbank flow. Unconfined streams were defined as having bankfull width to depth ratios greater than 10 , in many cases with multithreaded channels, and with more frequent connections between streamflows and flood-plain wetlands (Peterson and others, 2009). A dataset of 19 streams classified by Peterson and others (2009) in the Lower Flint Basin and 5 streams classified by fieldwork in the Potato Creek Basin (J. T. Peterson, oral commun., 2011; M.C. Freeman, oral commun., 2012) yielded no clear relations between the Peterson and others (2009) definition of stream confinement and remotely sensed valley width, channel width, valley width to channel width ratio, sinuosity, or surficial geology.

\section{Soil Erosion Potential and Urbanization Trends}

One of the broad goals of the SERAP project was to integrate lagged, multidecadal, and cumulative effects of disturbance and sediment routing on stream-channel dynamics to assess how changing channel morphology would interact with effects of changing runoff, water temperature, and water quality. Integrating these effects is especially challenging because it requires incorporating transport of sediment through a stream network, a process that may involve multiple episodes of deposition and remobilization. The cumulative effects of sediment routing on stream ecosystems may therefore lag substantially behind other climate or land-use effects. 
Incorporation of sediment routing into understanding of watershed dynamics has long been recognized as one of the grand challenges in geomorphology (Wolman, 1977). Although this report does not realize this goal, the SERAP project still seeks to develop understanding of lagged, long-term, and cumulative effects of land surface disturbance and sediment routing on channel dynamics to assess how changing channel morphology would interact with effects of changing runoff, water temperature, and water quality.

An intermediate step toward the broader goal of incorporating sediment routing through the ApalachicolaChattahoochee-Flint River Basin network was to develop a model of present-day soil-erosion potential. Although not necessarily predictive of how much sediment actually is delivered to the stream channel, the spatial distribution of erosion potential indicates sources of sediment that may eventually move through the stream network and affect downstream channels and aquatic ecosystems. Soil-loss predictions in this report are intended to evaluate the spatial distribution of relative potential disturbances from sediment in streams for the coarse-scale species-response model. In contrast to the urban area model, these predictions were not forecast through time because of concerns that cumulative uncertainties in the soil-erosion model parameters would result in low predictive power.

Performance of the soil-loss model under current conditions (based on 2001 land cover data) was assessed by general comparison to measured soil loss rates in the scientific literature. High values of soil loss have been documented in conventional agricultural lands based on sediment transport data (0.008-701 tons/acre/year with a mean of $52.5 \mathrm{tons} /$ acre/year, or $0.0006-52.5 \mathrm{~mm} / \mathrm{yr}$ with a mean of $3.97 \mathrm{~mm} / \mathrm{yr}$ ) (Montgomery, 2007), but soil-loss rate estimates vary based on methods (sediment transport fluxes or plotscale models) and area and time interval used for averaging. Although maximum rates calculated for any DEM cell in the Apalachicola-Chattahoochee-Flint River Basin are as much as 200 tons/acre/year, the number of cells with these extreme values are relatively small, and the HRU-averaged maximum rate is 4.5 tons/acre/year (fig. 25). The predicted soil erosion rates of $0.26-4.5$ tons/acre/year are in reasonable agreement with U.S. Department of Agriculture modeled estimates for the southeastern U.S. cropland of 3.4 tons/acre/year (U.S. Department of Agriculture, 2009a) and Georgia cropland of 4.7 tons/acre/year (U.S. Department of Agriculture, 2009b).

Low rates of soil erosion were estimated in HRUs in swampy wetlands of the Coastal Plain, in HRUs representing reservoirs, and in HRUs dominated by urban land-use reflected in a lower $\mathrm{C}$ values, consistent with impervious surfaces. Erosion rates in urbanizing areas potentially are underestimated because of soil erosion associated with new construction. Moderate values were observed in much of the Apalachicola-Chattahoochee-Flint River Basin, particularly in the lower and middle Flint River Basin and in the Coastal Plain sediments. High soil-loss values were associated with regions with both steeper hillslopes and extensive agricultural use predominately in the Blue Ridge and Piedmont.

The RUSLE soil erodibility model produced results within the expected ranges for various landscapes and land uses within the Apalachicola-Chattahoochee-Flint River Basin, yet predicting future stream-channel conditions with respect to sediment routing remains a challenge. Development of sediment-routing models at the drainage basin scale would require considerable effort including data collection for calibration and validation. Successful modeling would require capturing lagged and cumulative effects over long-term (decadal) time scales as sediment moves slowly downstream. Scientists have documented extensive flood-plain deposits of sediment from historical land-use changes in the 1800s and early 1900s in Georgia, indicating that long-term sediment routing does have potential to have a substantive effect on stream-channel condition, although understanding and predicting ongoing channel responses has proven difficult (Trimble, 1974; Jackson and others, 2005). A recent study of stream-channel stability of Georgia Piedmont streams based on trends at USGS streamgaging stations documented mixed patterns in streamchannel stability with about 40 percent of the streams in the analysis stable, 30 percent degrading, and around 15 percent aggrading or demonstrating variable trends (Riley and Jacobson, 2009). Sediment "fingerprinting" analysis in a Georgia Piedmont drainage basin just outside the Apalachicola-Chattahoochee-Flint River Basin demonstrated that erosion of legacy sediments from streambanks was responsible for 60 percent of measured suspended stream sediments (Mukundan and others, 2010). Ongoing remobilization of legacy sediments may create a contribution to suspended sediment loads that will be difficult to separate from the cumulative effects of climate and land-use change.

\section{Stream Classification}

Many approaches exist for river classification, and use of any particular system should be based on how well the classification fits the intended application (Kondolf and others, 2003). For the Apalachicola-Chattahoochee-Flint River Basin project, stream classification provides a basis for lumping and splitting segments and reaches with common sets of physical characteristics, which are assumed to have common and distinct sets of responses to climate and land-use changes.

Reach-scale river classification, similar to ecosystem classification, has been addressed typically through one of two broad approaches, integrated or component (Driscoll and others, 1984). In an integrated approach, predetermined classification systems are assigned to divide a river into physically meaningful classification units. The most frequently cited system is the geomorphic classification by Rosgen (Rosgen, 1996). Integrated, deductive classification systems like the Rosgen (1996) system are based on assumptions that wellunderstood processes determine stream-channel form and that 
stream-channel form is indicative of geomorphic processes, and by extension, ecological processes. A contrasting approach is based on inductive reasoning and involves measuring a broad suite of stream-geomorphology characteristics, followed by application of multivariate statistical techniques to extract naturally occurring clusters. Statistical, or component, classification systems have advantages in minimizing dependence on prior assumptions; however, even these statistical approaches make some basic assumptions about geomorphic form and process. A component approach was selected in the Apalachicola-Chattahoochee-Flint River Basin for its value in providing an objective classification and for its ability to be applied through statistical models of landscape-scale characteristics.

The size and complexity of the ApalachicolaChattahoochee-Flint River Basin made it impossible to apply detailed measurements to all the streams in the basin. As a substitute, we used readily available, landscape-scale GIS data to associate channel-scale habitat characteristics with landscape-scale characteristics. This practice has been used to delineate channel types related to wetland development in Italy (Angiolini and others, 2011), stream habitats in the Midwest United States (Brenden and others, 2008a; Brenden and others, 2008b), and in Europe (Bizzi and Lerner, 2012). This classification, based on available basin-wide data, did not provide the desired amount of information content on stream incision and flood plain connectivity; however, the classification's integration of multiple geomorphic attributes did ultimately provide explanatory value for the occurrence of two endemic species of fish in the Apalachicola-ChattahoocheeFlint River Basin.

\section{Biological Application}

The stream classification was tested using pairs of data for two species of darters and two species of minnows. In each case, one species had a broad distribution throughout river drainages in the southeastern Gulf Coast and the other species was endemic to the Apalachicola-Chattahoochee-Flint River Basin with a much more restricted distribution. The two sets of paired species generally were documented in different classes and used stream classes in different proportions than those available in the Apalachicola-Chattahoochee-Flint River Basin. For the Halloween and blackbanded darters, the classification model was a more predictive model of species occurrence than the model based on individual geomorphic variables. Species occurrence percentages suggest the classification model is useful for predicting the distribution patterns of the bluestripe shiner as well. The classification could be tested further using other species. Mussel distribution data also could provide information on the utility of the classification for other aquatic species of concern in the ApalachicolaChattahoochee-Flint River Basin. Other applications of the Apalachicola-Chattahoochee-Flint River Basin reach-scale classification include the designing of sampling or monitoring schemes and the prediction and testing of locations in the Apalachicola-Chattahoochee-Flint Basin that could be suitable habitat for a species of interest.

Prototype metapopulation models developed in the lower Flint River used a stream classification developed for the lower Flint River that integrated surficial geology (Ocala Limestone or Fall Line Hills) and stream morphology (confined or unconfined). A metapopulation model for Potato Creek classified segments as "unconfined" if more than 10 percent of the adjacent drainage area (HRU) had zero slope estimated from a 30-m DEM. Metapopulation model results for Potato Creek suggest outcomes are moderately sensitive to stream-channel misclassification and would be more accurate with improved classification (Freeman and others, 2012). The degree of stream confinement is important in classification, although complicated by varying definitions. Essentially, stream-channel confinement, as defined by Peterson and others (2009), represents incised channels confined by alluvium, a situation that can be located in a wide or relatively narrow valley setting. This scale of flood-plain incision was not obtainable from the 30-m scale DEM and drainage-basin area driven channel geometry calculations used in this study. Lidar data or field survey stream-channel cross sections and field assessments could be used to develop a stream classification at the scale defined in Peterson and others (2009). The valley-scale stream confinement metric developed in this report does not conform to the Peterson and others (2009) definition and does not resolve flood-plain connectivity. Instead, the valley-scale metric resolves if an appreciable extent of alluvial flood plain is present, or conversely, if the stream is likely to be interacting with bedrock in valley walls.

\section{Application to Climate and Land-Use Modeling}

The classification was designed with the potential to be linked to climate-driven hydrologic and biological models and provide a basis for delineating stream classes with fundamentally different biophysical capacities and possible sensitivities to climate and land-use changes. The classification serves to delineate reaches of Apalachicola-Chattahoochee-Flint River Basin streams that should have similar ability to mediate hydrologic effects in contributing drainage basins. In this sense, the classification serves as a filter between hydrologic effects on streams and how hydrology could affect physical habitats occupied by stream biota.

An ongoing challenge is to predict how stream conditions may change, resulting in different future distribution of stream classes, or transitions from one class to another. Complex, and potentially lagged, interactions among flow regime, sediment routing, and local hydraulics make it difficult to predict specific transitions with any precision; however, the current (2013) distribution of stream classes, contemporary distribution of soil-erosion potential, and calculations of the future 
spatial distribution of stream power from hydrologic models provide a basis for inferring sensitivity to climate- or landuse-induced changes in stream condition. For example, rivers in wide flood plains where there is space for stream channels to migrate would be expected to be more vulnerable to changes in channel form, bank erosion, and scour or deposition compared to narrow mountain streams, which lack room for storage in their flood plains. Streams in wide flood plains would be expected to adjust to increased or decreased fine sediment supply from upstream, whereas the narrow mountain streams would not. Similarly, different clusters may be more or less vulnerable to stream-temperature changes depending on how channel adjustments and riparian land-use practices alter riparian shading. Although potential dynamic changes in stream-channel form present additional uncertainties to predictions of how climate and land-use change would propagate to stream biota, the SERAP classification is expected to retain utility for stratifying and understanding future changes. Future applications could use calculations of changes in unit stream power and watershed modeling results for varying climate and land-use scenarios that could provide a mechanism for predicting parts of the stream network where climate-induced changes in flow regime would be expected to cause streamchannel change.

\section{Summary}

This report documents development and testing of a stream network and classification for application to the Southeast Regional Assessment Project (SERAP). The SERAP used downscaled general circulation models to develop assessments of how climate and associated land-use changes are likely to affect ecosystems, land cover, and priority species in the southeastern United States. The SERAP aquatic and hydrologic dynamics modeling efforts involving multiscale watershed, stream-temperature, and fish-occupancy models all utilize the same stream network and classification.

The stream network generated for the SERAP aquatic models was generated from a 30-meter scale digital elevation model and has a similar drainage density and structure to the medium-resolution $(1: 100,000)$ NHD stream network. Every stream reach in the network is populated with individual geomorphic attributes. Attributes at the reach scale include upstream drainage basin area, gradient, channel width, valley width, Strahler and Shreve stream order, stream power, and measures of stream confinement. Channel width was modeled as a function of drainage-basin area using regional streamchannel geometry relations. Valley width was modeled as a function of drainage-basin area from regional channel geometry relations of bankfull width and depth intersected with valley walls in the 30-m DEM. Streams less than $10 \mathrm{~m}$ wide or with a ratio of valley width to bankfull width that was less than 4 were designated as confined, whereas all others were designated as unconfined.
Two stream-network datasets were developed for application in the SERAP. A segment-based network aggregated from tributary junction to tributary junction was developed for seven selected drainage basins to support detailed fish and mussel metapopulation modeling and contains additional attributes on barriers or impoundments and stream isolation. The segment network also is attributed with percent urban area for the watershed upstream from the stream segment for each decade from 2010-2100 (probability from models with 50 percent or greater probability of urbanization).

A reach-based network (with segments sub-divided at geomorphic boundaries) was classified using multivariate clustering of channel width, valley width, and mean reach gradient as variables. The resulting classification is multiscale, with 6-cluster and 12-cluster classifications for the entire Apalachicola-Chattahoochee-Flint River Basin. The classification was tested for biological relevance using species occurrence of two species of darters and two species of minnows, the bluestripe shiner and blacktail shiner in the ApalachicolaChattahochee-Flint River Basin. The classification contributed to models of the distributions of these species.

For a coarse-scale species-response model for the entire Apalachicola-Chattahoochee-Flint, the stream-network information was aggregated and summarized by subbasin, or Hydrologic Response Units. Soil erodibility (estimated using the Revised Universal Soil Loss Equation) and other geomorphic variables, including stream size, confinement, and gradient, were summarized at the HRU level for the coarse-scale species-response modeling effort, providing a useful template for predicting and understanding multidecadal landscape responses to climate and land-use change. This report documents methods applied for the entire ApalachicolaChattahoochee-Flint River Basin and seven subbasins to derive stream delineations and classifications to support a coordinated interdisciplinary multimodel project.

\section{Acknowledgments}

Funding for this project was provided by the U.S. Geological Survey (USGS) through the Southeast Regional Assessment Project (SERAP) and the Department of the Interior's Southeast Climate Science Center. Gerald McMahon from the Southeast Climate Science Center provided funding for this report.

Jeff Riley (USGS Georgia Water Science Center) and Milan Thorsby (USGS Columbia Environmental Research Center) collected field data at 59 field sites in the Apalachicola-Chattahoochee-Flint Basin. Jeff Riley contributed an analysis of gage information for the Apalachicola-ChattahoocheeFlint Basin to examine channel dimensions. John W. Jones (USGS, Reston, Virginia) provided waterbody map layers and valuable insight on the dynamics of small waterbodies in the Apalachicola-Chattahoochee-Flint River Basin and model subbasins. Adam Terando, Curtis Belyea, Alexa McKerrow, and 
Jennifer Costanza at the Biodiversity and Spatial Information Center at North Carolina State University provided the Urban Growth modeling data. Many individuals within the SERAP aquatic dynamics modeling group contributed to this project including Brian Hughes, Melinda Daniels, Jim Peterson, Jacob LaFontaine, Lauren Hay, John W. Jones, Roland Viger, Steve Markstrom, and Colin Shea.

\section{References Cited}

Anderson, G.B., Freeman, M.C., Freeman, B.J., Straight, C.A., Hagler, M.M., and Peterson, J.T., 2012, Dealing with uncertainity when assessing fish passage through culvert road crossings: Environmental Management, v. 50, p. 462-477.

Angiolini, C., Nucci, A., Frignani, F., and Landi, M., 2011, Using multivariate analyses to assess effects of fluvial type on plant species distribution in a mediterranean river: Wetlands, v. 31, no. 1, p. 167-177. [Also available at http:// dx.doi.org/10.1007/s13157-010-0118-7.]

Beechie, T.J., Liermann, M., Pollock, M.M., Baker, S., and Davies, J., 2006, Channel pattern and river-floodplain dynamics in forested mountain river systems: Geomorphology, v. 78, p. 124-141.

Belyea, C.M., and Terando, A.J., 2012, Urban growth modeling for the sambi designing sustainable landscapes project, in Biodiversity and Spatial Information Center, N.C.S.U.: Raleigh, N.C., Biodiversity and Spatial Information Center, accessed June 1, 2012, at http://www.basic.ncsu.edu.

Benda, L., Miller, D., Andras, K., Bigelow, P., Reeves, G., and Michael, D., 2007, Netmap-A new tool in support of watershed science and resource management: Forest Science, v. 53, no. 2, p. 206-219.

Bizzi, S., and Lerner, D.N., 2012, Characterizing physical habitats in rivers using map-derived drivers of fluvial geomorphic processes: Geomorphology, v. 169-170, no. 0, p. 64-73. [Also available at $h t t p: / / d x . d o i . o r g / 10.1016 / j$. geomorph.2012.04.009.]

Brenden, T.O., Wang, L., and Seelbach, P.W., 2008a, A river valley segment classification of michigan streams based on fish and physical attributes: Transactions of the American Fisheries Society, v. 137, no. 6, p. 1621-1636. [Also available at $h t t p: / / d x$.doi.org/10.1577/T07-166.1.]

Brenden, T.O., Wang, L., Seelbach, P.W., Clark, R.D., Jr., Wiley, M.J., and Sparks-Jackson, B.L., 2008b, A spatially constrained clustering program for river valley segment delineation from GIS digital river networks: Environmental Modelling \& Software, v. 23, no. 5, p. 638-649.
Brierley, G.J., and Fryirs, K.A., 2005, Geomorphology and river management applications of the river styles framework Blackwell Publishing, $387 \mathrm{p}$.

Brim Box, J., and Williams, J.D., 2000, Unionid mollusks of the Apalachicola basin in Alabama, Florida, and Georgia: Bulletin of the Alabama Museum of Natural History, v. 21, p. 1-143.

Burnham, K.P., and Anderson, D.R., 2002, Model selection and multi-model inference-A practical informationtheoretic approach ( $2 \mathrm{~d}$ ed.), New York, Springer-Verlag, $488 \mathrm{p}$.

Carlisle, D.M., Wolock, D.M., and Meador, M.R., 2010, Alteration of streamflow magnitudes and potential ecological consequences - A multiregional assessment: Frontiers in Ecology and the Environment, v. 9, no. 5, p. 264-270. [Also available at $h t t p: / / d x$.doi.org/10.1890/100053.]

Couch, C.A., Hopkins, E.H., and Hardy, P.S., 1996, Influences of environmental settings on aquatic ecosystems in the Apalachicola-Chattahoochee-Flint River Basin: U.S. Geological Survey Water-Resources Investigations Report 95-4278, 58 p.

Crawford, R.W., 1956, A study of the distribution and taxonomy of the percid fish percina nigrofasciata (agazziz): Tulane Studies in Zoology, v. 4, p. 1-55.

Dalton, M.S., and Jones, S.A., eds., 2010, Southeast regional assessment project for the national climate change and wildlife science center: U.S. Geological Survey Open-File Report 2010-1213, 38 p.

Dietrich, W.E., Wilson, C.J., Montogomery, D.M., and McKean, J., 1993, Analysis of erosion thresholds, channel networks, and landscape morphology using a digital terrain model: Journal of Geology, v. 101, p. 259-278.

Doll, B.A., Wise-Frederick, D.E., Buckner, C.M., Wilkerson, S.D., Harman, W.A., Smith, R.E., and Spooner, J., 2002, Hydraulic geometry relationships for urban streams throughout the Piedmont of North Carolina: Journal of the American Water Resources Association, v. 38, no. 3, p. 641-651.

Driscoll, R.S., Merkel, D.L., Radloff, D.L., Snyder, D.E., and Hagihara, J.S., 1984, An ecological land classification framework for the United States: U.S. Department of Agriculture Forest Service, Miscellaneous Publication 1439, $56 \mathrm{p}$.

Fenneman, N.M., 1938, Physiography of eastern United States: New York, McGraw-Hill, 691 p. 
Freeman, M.C., Buell, G.R., Hay, L.E., Hughes, W.B., Jacobson, R.B., Jones, J.W., Jones, S.A., LaFontaine, J.H., Odom, K.R., Peterson, J.P., Riley, J.W., Schindler, J.S., Shea, C.P., and Weaver, J.D., 2012, Linking river management to species conservation using dynamic landscape-scale models: River Research and Applications, v. 29, no. 7, p. 906-918. [Also available at $h t t p: / / d x$.doi.org/10.1002/rra.2575.]

Freeman, M.C., Freeman, B.J., Burkhead, N.M., and Straight, C.A., 2008, A new species of percina (perciformes: Percidae) from the Apalachicola River drainage, southeastern United States: Zootaxa, v. 1963, p. 25-42.

Freeman, M.C., Peterson, J.T., Elliott, C.M., Shea, C.P., and Hagler, M.M., 2011, Using species traits and geomorphic characteristics to condition coarse-resolution assessment of climate change effects on aquatic species, in Ecological Society of America, Austin, Tex., 2011, Proceeedings of the Ecological Society of America: Austin, Tex., p. 91.

Frimpong, E.A., and Angermeier, P.L., 2009, Fishtraits-A database of ecological and life-history traits of freshwater fishes of the United States: Fisheries, v. 34, no. 10, p. 487-495.

Frissell, C.A., Liss, W.J., Warren, C.E., and Hurley, M.D., 1986, A hierarchical framework for stream habitat classification-Viewing streams in a watershed context: Environmental Management, v. 10, no. 2, p. 199-214.

Garrity, C.P., and Soller, D.R., 2009, Database of the geologic map of North America; adapted from the map by J.C. Reed, Jr., and others (2005): U.S. Geological Survey Data Series 424 .

Georgia Soil and Water Conservation Commission, 2000, Manual for erosion and sediment control in Georgia: Georgia Soil and Water Conservation Commission, $13 \mathrm{p}$.

Gesch, D.B., ed., 2007, The National Elevation Dataset (2 d.): Bethesda, Maryland, Americal Society for Photogrammetry and Remote Sensing, p. 99-118.

Gesch, D.B., Oimoen, M., Greenlee, S., Nelson, C., Steuck, M., and Tyler, D., 2002, The National Elevation Dataset: Photogrammetric Engineering and Remote Sensing, v. 68, no. 1, p. 5-11.

Glickauf, S., Harman, W.A., Bevington, S., and Gilland, K., 2007, The development of bankfull hydraulic geometry relationships for streams of the Georgia Coastal Plain, in Georgia Water Resources Conference, Athens, Ga., 2007, Proceedings: Athens, Ga., University of Georgia, variously paged.

Guillory, S.V., 1976, Apparent absence of percina nigrofasciata from the Mississippi Valley: Copeia, p. 804-805.
Harman, W.A., Jennings, G.D., Patterson, J.M., Clinton, D.R., Slate, L.O., Jessup, A.G., Everhart, J.R., and Smith, R.E., 1999, Bankfull hydraulic geometry relationships for North Carolina streams, in American Water Resources Association Wildland Hydrology Conference, Bozeman, Mont., American Water Resources Association Wildland Hydrology Proceedings, variously pagninated.

Homer, C., Huang, C., Yang, L., Wylie, B., and Coan, M., 2004, Development of a 2001 national landcover database for the United States: Photogrammetric Engineering and Remote Sensing, v. 70, no. 7, p. 829-840.

Hornby, D.D., 2010, Rivex (version 9.1) software: Southampton, United Kingdom, available at $h t t p: / / w w w . R i v e x . C o . U k$.

Jackson, C.R., Martin, J.K., Leigh, D.S., and West, L.T., 2005, A southeastern Piedmont watershed sediment budget-Evidence for a multi-millennial agricultural legacy: Soil and Water Conservation, v. 60, no. 6, p. 298-310.

Jacobson, R.B., and Coleman, D.J., 1986, Stratigraphy and recent evolution of Maryland Piedmont flood plains: American Journal of Science, v. 286, no. 8, p. 617-637.

Jantz, C.A., and Goetz, S.J., 2005, Analysis of scale dependencies in an urban-land-use change model: International Journal of Geographical Information Science, v. 19, no. 2, p. $217-241$.

Jenson, S., and Domingue, J., 1988, Extracting topographic structure from digital elevation data for geographic information system analysis: Photogrammetric engineering and remote sensing, v. 54, no. 11, p. 1593-1600.

Johnson, P.A., and Fecko, B.J., 2008, Regional channel geometry equations - A statistical comparison for physiographic provinces of the eastern United States: River Research and Applications, v. 24, p. 823-824. [Also available at $h t t p: / /$ dx.doi.org/DOI:10.1002/rra.1080.]

Knighton, D., 1998, Fluvial forms and processes-A new perspective: New York, Oxford University Press Inc., 383 p.

Kondolf, G.M., Montogomery, D.M., Piégay, H., and Schmitt, L., 2003, Geomorphic classification of rivers and streams, in Kondolf, G.M., and Piégay, H., eds., Tools in fluvial geomorphology: Chichester, England, John Wiley and Sons, p. 171-204.

LaFontaine, J.H., Hay, L.E., Viger, R.J., Markstrom, S.L., Regan, S., Elliott, C.M., and Jones, J.W., 2013, Application of the precipitation-runoff modeling system (PRMS) in the Apalachicola-Chattahoochee-Flint River Basin in the southeastern United States, U.S. Geological Survey Scientific Investigations Report 2013-5162, 118 p.

Leavesley, G.H., 1983, Precipitation-runoff modeling system-User's manual: U.S. Geologcial Survey WaterResources Investigations Report 83-4238, 207 p. 
Leopold, L.B., and Wolman, M.G., 1957, River channel patterns - Braided, meandering, and straight: U.S. Geological Survey Professional Paper 252-B, 85 p.

Light, H.M., Darst, M.R., and Grubbs, J.W., 1998, Aquatic habitats in relation to river flow in the Apalachicola River floodplain, Florida: U.S. Geological Survey Professional Paper 1594, $77 \mathrm{p}$.

Lydeard, C., and Mayden, R.L., 1995, A diverse and endangered aquatic ecosystem of the Southeast United States: Conservation Biology, v. 9, p. 800-805.

Marcot, B.G., Holthausen, R.S., Raphael, M.G., Rowland, M.M., and Wisdom, M.J., 2001, Using bayesian belief networks to evaluate fish and wildlife population viability under land management alternatives from an environmental impact statement: Forest Ecology and Management, v. 153, p. 29-42.

McCargo, J.W., and Peterson, J.T., 2010, An evaluation of the influence of seasonal base flow and geomorphic stream characteristics on coastal plain stream fish assemblages: Transactions of the American Fisheries Society, v. 139, p. 29-48.

McManamay, R.A., Orth, D.J., Dolloff, C.A., and Frimpong, E.A., 2011, A regional classification of unregulated stream flows - Spatial resolution and hierarchical frameworks: River Research and Applications. [Also available at $h t t p: / /$ dx.doi.org/10.1002/rra.1493.]

Miller, D., 2008, Programs for dem analysis: Earth Systems Institute, $20 \mathrm{p}$.

Mitsova, H., Hofierka, J., Zloca, M., and Iverson, L.R., 1996, Modeling topographic potential for erosion using a gis: International Journal of Geographic Information Systems, v. 10 , no. 5 , p. $629-641$.

Mitsova, H., and Mitas, L., 2001, Multiscale soil erosion simulations for land use management, in Harmon, R.S., and Doe, W.W., eds., 2001: Landscape Erosion and Evolution Modeling, Springer, U.S., New York, New York, p. 321-347.

Mohamoud, Y.M., and Parmar, R.S., 2006, Estimating streamflow and associated hydraulic geometry, the Mid-Atlantic region, USA: Journal of the American Water Resources Association, v. 42, no. 3, p. 755-768.

Montgomery, D.M., 1999, Process domains and the river continuum: Journal of the American Water Resources Association, v. 35 , no. 2, p. 397-410.

Montgomery, D.M., 2007, Soil erosion and agricultural sustainability: Proceedings of the National Academy of Sciences of the United States of America, v. 104, no. 33, p. 13, 628-13272.
Montgomery, D.R., and Dietrich, W.E., 1992, Channel initiation and the problem of landscape scale: Science, v. 255, p. $826-830$.

Mukundan, R., Radcliffe, D.E., Ritchie, J.C., Risse, L.M., and McKinley, R.A., 2010, Sediment fingerprinting to determine the source of suspended sediment in a southern Piedmont stream: Journal of Environmental Quality, v. 39, p. 1328-1337.

Nanson, G., and Croke, J., 1992, A genetic classification of floodplains: Geomorphology, v. 4, no. 6, p. 459-486.

NatureServe, 2007, International ecological classification standard--Terrestrial ecological classifications: Arlington, Virginia, NatureServe Central Databases, 443 p., accessed April 1, 2012, at http://www.basic.ncsu.edu/segap/datazip/ region/se_lc_systems_descriptions.pdf.

Olivero, A.P., and Anderson, M.G., 2008, Northeast aquatic habitat classification system: The Nature Conservancy, Eastern Regional Office, 88 p.

Patric, J.H., Evans, J.O., and Helvey, J.D., 1984, Summary of sediment yield data from forested lands in the United States: Journal Of Forestry, p. 101-104.

Paul, M.J., and Meyer, J.L., 2001, Streams in the urban landscape: Annual Review of Ecology and Systematics, v. 32, no. 1, p. 333-365. [Also available at $h t t p: / / d x . d o i$. org/10.1146/annurev.ecolsys.32.081501.114040.]

Peterson, J.T., Freeman, M.C., LaFontaine, J.H., Hay, L., Jones, J.W., Jacobson, R.B., Elliott, C.M., and Hughes, W.B., 2011a, An evaluation of fragmentation on stream fish communities using spatially-explicit metapopulation models, in American Fisheries Society Annual Meeting, Seattle Wash., 2011: Procedings, Seattle, Wash., variously paged.

Peterson, J.T., Freeman, M.C., LaFontaine, J.H., Jacobson, R.B., Elliott, C.M., Hay, L., and Jones, J.W., 2011b, Using structured decision making and adaptive management to reduce critical uncertanites in water resource decisions: Examples from the ACF basin, in American Fisheries Society Annual Meeting, Seattle Wash., 2011: Procedings, Seattle, Wash., variously paged.

Peterson, J.T., Hay, L., Odom, K., Hughes, W.B., Jacobson, R.B., Jones, J., and Freeman, M., 2010, Multi-resolution assessment of potential climate change effects on biological resources: Aquatic and hydrologic dynamics, in Dalton, M.S., and Jones, S.A., eds., Southeast regional assessment project for the National Climate Change and Widlife Science Center, U.S. Geological Survey Open-File Report 2010-1213, p. 25-29. 
Peterson, J.T., Jackson, C.R., Shea, C.P., and Li, G., 2009, Development and evalulation of a stream channel classification for estimating fish responses to changing streamflow: Transactions of the American Fisheries Society, v. 138, p. 1123-1137.

Poff, N.L., 1997, Landscape filters and species traitsTowards mechanistic understanding and prediction in stream ecology: Journal of the North American Benthological Society, v. 16, no. 2, p. 391-409.

Poff, N.L., Allan, J.D., Bain, M.B., Karr, J.R., Prestegaard, K.L., Richter, B.D., Sparks, R.E., and Stromberg, J.C., 1997, The natural flow regime: Bioscience, v. 47, p. 769-784.

Poff, N.L., Richter, B.D., Arthington, A.H., Bunn, S.E., Naiman, R.J., Kendy, E., Acreman, M., Apse, C., Bledsoe, B., Freeman, M.C., Henriksen, J.A., Jacobson, R.B., Kennen, J.G., Merritt, D.M., O'Keefe, J.H., Olden, J.D., Rogers, K., Tharme, R.E., and Warner, A., 2009, The ecological limits of hydrologic alteration (ELOHA) - A new framework for developing regional environmental flow standards: Freshwater Biology, v. 55, p. 147-170. [Also available at http://dx.doi.org/10.1111/j.1365-2427.2009.02204.x.]

R Core Team, 2009, R-A language and environment for statistical computing: R Foundation for Statistical Computing, Vienna, Austria, available at http:/www.R-project.org/.

Rabeni, C.F., and Sowa, S.P., 2002, A landscape approach to managing the biota of streams and rivers, in Liu, J., and Taylor, W., eds., Integrating landscape ecology into natural resource management: Cambridge, Cambridge University Press, p. 114-142.

Reidy Liermann, C.A., Olden, J.D., Beechie, T.J., Kennard, M.J., Skidmore, P.B., Konrad, C.P., and Imaki, H., 2011, Hydrogeomorphic classification of Washington State rivers to support emerging environmental flow management strategies: River Research and Applications, v. 28, no. 9, p. 1-19.

Renard, K.G., Foster, G.R., Weesies, G.A., McCool, D.K., and Yoder, D.C., 1997, A guide to conservation planning with the revised universal soil loss equation (RUSLE): U.S. Department of Agriculture, Agricultural Handbook no. 703, $383 \mathrm{p}$.

Rieman, B., Peterson, J.T., Clayton, J., Howell, P., Thurow, R., Thompson, W., and Lee, D., 2001, Evaluation of potential effects of federal land management alternatives on trends of salmonids and their habitats in the interior Columbia River basin: Forest Ecology and Management, v. 153, p. 43-62.

Riley, J.W., and Jacobson, R.B., 2009, Long-term stage, stageresidual, and width data for streams in the Piedmont physiographic region, Georgia: U.S. Geological Survey Open-File Report 2009-1205, 46 p.
Rosgen, D., 1996, Applied river morphology, Pagosa Springs, Colo., Wildland Hydrology, 352 p.

Ruiz, J., and Peterson, J.T., 2007, An evaluation of the relative influence of spatial, statistical, and biological factors on the accuracy of stream fish species presence models: Transactions of the American Fisheries Society, v. 136, p. 1640-1653.

Schmitt, L., Maire, G., Nobelis, P., and Humbert, J., 2007, Quantitative morphodynamic typology of rivers-A methodological study based on the French Upper Rhine basin: Earth Surface Processes and Landforms, v. 32, no. 11, p. 1726-1746.

Shreve, R.L., 1967, Infinite topographically random channel networks: Journal of Geology, v. 75, p. 397-414.

Snelder, T., Biggs, B.J.F., and Woods, R.A., 2005, Improved eco-hydrological classification of rivers: River Research and Applications, v. 21, p. 609-628.

Snelder, T.H., Dey, K.L., and Leathwick, J.R., 2007, A procedure for making optimal selection of input variables for multivariate environmental classifications: Conservation Biology, v. 21, no. 2, p. 365-375.

Soil Survey Staff, 2006, U.S. General soil map (STATSGO2) database: U.S. Department of Agriculture, Natural Resources Conservation Service, accessed October 2012, at http://SoilDataMart.nrcs.usda.gov/.

Soil Survey Staff, 2012, Spatial and tabular data of soil surveys for counties in Georgia, Florida, Alabama, and Virginia, soil survey geographic (SSURGO) database: U.S. Department of Agriculture, Natural Resources Conservation Service, accessed October 2012, at http://SoilDataMart. nrcs.usda.gov/.

Strahler, A.N., 1952, Dynamic basis of geomorphology: Geological Society of America Bulletin, v. 63, p. 923-938.

Systat Software, Inc., 2012, Statistics I: Richmond, Calif., Systat, Inc., 493 p.

Thorp, J.H., Thoms, M.C., and Delong, M.D., 2006, The riverine ecosystem synthesis-Biocomplexity in river networks across space and time: River Research and Applications, v. 22 , no. 2 , p. $123-147$.

Torak, L.J., and Painter, J.A., 2006, Geohydrology of the lower Apalachicola-Chattahoochee-Flint River Basin, southwestern Georgia, northwestern Florida, and southeastern Alabama: Scientific Investigations Report 2006-5070, $80 \mathrm{p}$.

Trimble, S.W., 1974, Man-induced soil erosion on the southern Piedmont, 1700-1970: Ankeny, Iowa, Soil Conservation Society of America, $80 \mathrm{p}$. 
U.S. Army Corps of Engineers, 2010, National inventory of dams: U.S. Army Corps of Engineers database, accessed February 2012, at http://crunch.Tec.Army.Mil/nidpublic/ webpages/nid.Cfm.

U.S. Congress, 1874, A report on the survey of the Flint and Chattahoochee in Florida: Executive Document No. 65, Washington D. C, United States House of Representatives, $37 \mathrm{p}$.

U.S. Congress, 1910, A report on the river and harbor bill by the committee on commerce, Washington, D.C., United States Senate: Government Printing Office, report no. 527, $17 \mathrm{p}$.

U.S. Department of Agriculture, Natural Resource Conservation Service, 2009a, 2007 National resources inventorySoil erosion on cropland: Natural Resources Conservation Service, Washington, D.C., and Center for Survey Statistics and Methodology, Iowa State University, Ames, Iowa, 29 p. [Also available at $h t t p: / / w w w . n r c s . u s d a . g o v / w p s / p o r t a l / n r c s /$ detail/national/technical/nra/nri/?cid=stelprdb1041887.]

U.S. Fish and Wildlife Service, 1994-2000, National Wetlands Inventory website: U.S. Department of the Interior, Fish and Wildlife Service, accessed December 15, 2009, at http:// www.fws.gov/wetlands/.

Vannote, R.L., Minshall, G.W., Cummins, K.W., Sedell, J.R., and Gushing, E., 1980, The river continuum concept: Canadian Journal of Fisheries and Aquatic Sciences, v. 37, p. 130-137.

Viger, R.J., Hay, L.E., Jones, J.W., and Buell, G.R., 2010, Effects of including surface depressions in the application of the precipitation-runoff-modeling system in the Upper Flint River Basin, Georgia: U.S. Geological Survey Scientific Investigations Report 2010-5062, 36 p.

Viger, R.J., Hay, L.E., Markstrom, S.L., Jones, J.W., and Buell, G.R., 2011, Hydrologic effects of urbanization and climate change in the Flint River Basin, Georgia: Earth Interactions, v. 15, 1-25.

Walter, R.C., and Merritts, D.J., 2008, Natural streams and the legacy of water-powered mills: Science, v. 319, no. 5861, p. 299-304.
Walters, D.M., Leigh, D.S., Freeman, M.C., Freeman, B.J., and Pringle, C.M., 2003, Geomorphology and fish assemblages in a Piedmont river basin, U.S.A.: Freshwater Biology, v. 48, p. 1950-1970.

Wang, L., Infante, D., Esselman, P., Cooper, A., Wu, D., Taylor, W., Beard, D., Whelan, G., and Ostroff, D., 2011, A hierarchical spatial framework and database for the national river fish habitat condition assessment: Fisheries, v. 36, no. 9, p. 436-449.

Ward, G.M., Harris, P.M., and Ward, A.K., 2005, Gulf coast rivers of the southeastern United States, in Benke, A.C., and Cushing, C.E., eds., Rivers of North America: Elsevier, p. 125-178.

Warren, M.L., Burr, B.M., Walsh, S.J., Bart, H.L., Cashner, R.C., Etnier, D.A., Freeman, B.J., Kuhajda, B.R., Mayden, R.L., Robison, H.W., Ross, S.T., and Starnes, W.C., 2000, Diversity, distribution, and conservation status of the native freshwater fishes of the southern United States: Fisheries, v. 25 , no. 10 , p. $7-31$.

Wenger, S.J., Peterson, J.P., Freeman, M.C., Freeman, B.J., and Homans, D.D., 2008, Stream fish occurrence in response to impervious cover, historic land use, and hydrogeomorphic factors: Canadian Journal of Fisheries and Aquatic Sciences, v. 65, p. 1250-1264.

White, J.Q., Pasternack, G.B., and J., M.H., 2010, Valley width variation influences riffle-pool location and persistence on a raidly incising gravel-bed river: Geomorphology, v. 121, p. 206-221.

Wilkinson, L., Engelman, L., Corter, J., and Coward, M., 2004, Cluster analysis - Statistics i: Richmond, California, Systat Inc., p. 57-91.

Wischmeier, W.H., and Smith, D.D., 1978, Predicting rainfall erosion losses-A guide to conservation planning: U.S. Department of Agriculture handbook 537, $58 \mathrm{p}$.

Wolman, M.G., 1977, Changing needs and opportunities in the sediment field: Water Resources Research, v. 13, p. 50-54. 

Appendixes 
Table 1-1. Reach-scale layer attributes.

[FID, feature identification number; m, meters; PRMS, Precipitation Runoff Modeling System; m, meters]

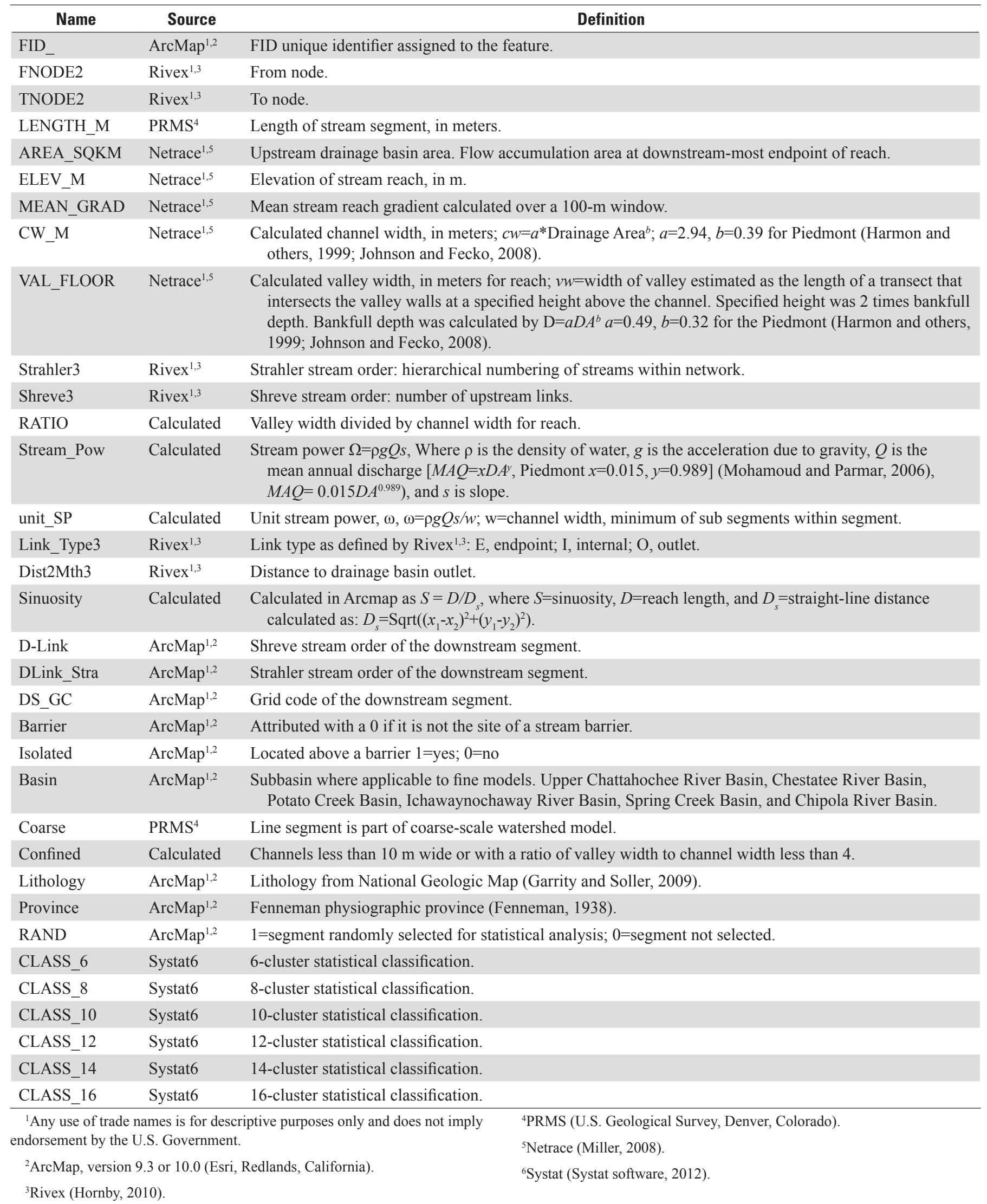


Table 2-1. Segment-scale layer attributes.

[FID, feature identification number; PRMS, Precipitation Runoff Modeling System; m, meters; $\mathrm{m}^{2}$, square meters; \%, percent]

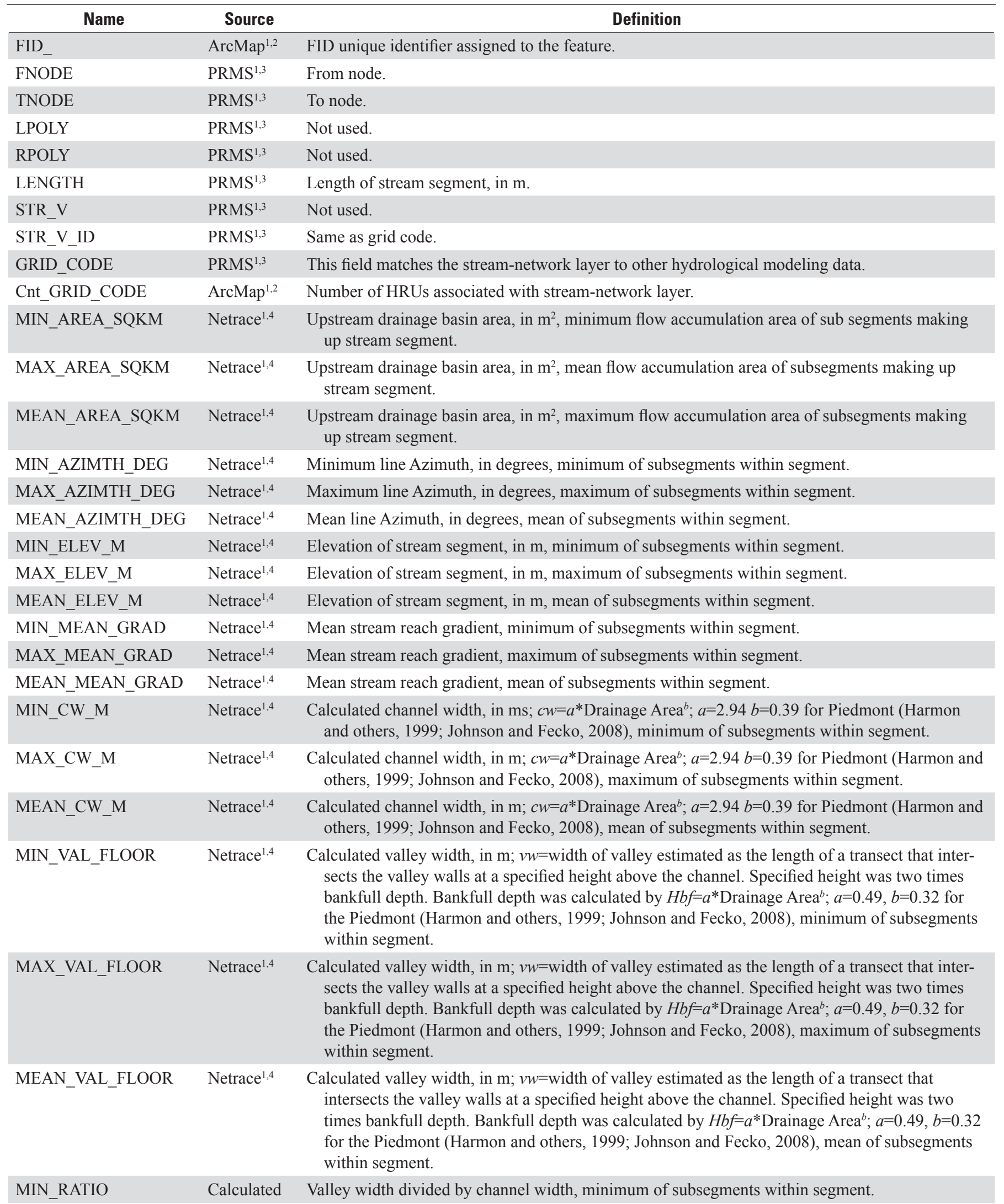


Table 2-1. Segment-scale layer attributes.-Continued

[FID, feature identification number; PRMS, Precipitation Runoff Modeling System; m, meters; $\mathrm{m}^{2}$, square meters; \%, percent]

\begin{tabular}{|c|c|c|}
\hline Name & Source & Definition \\
\hline MAX_RATIO & Calculated & Valley width divided by channel width, maximum of subsegments within segment. \\
\hline MEAN_RATIO & Calculated & Valley width divided by channel width, mean of subsegments within segment. \\
\hline MAX_STREAM_POW & Calculated & $\begin{array}{l}\text { Stream power } \Omega=\rho g Q s \text {, where } \rho \text { is the density of water, } g \text { is the acceleration due to gravity, } Q \text { is } \\
\text { the mean annual discharge }\left[M A Q=x D A^{y} \text { Piedmont } x=0.015 y=0.989 \text { (Mohamoud and Parmar, }\right. \\
\text { 2006), } M A Q=0.015 D A^{0.989} \text { ), and s is slope, maximum of subsegments within segment. }\end{array}$ \\
\hline MEAN_STREAM_POW & Calculated & $\begin{array}{l}\text { Stream power } \Omega=\rho g Q s \text {, where } \rho \text { is the density of water, } g \text { is the acceleration due to gravity, } Q \text { is } \\
\text { the mean annual discharge }\left[M A Q=x D A^{\mathrm{y}} \text { Piedmont } x=0.015 y=0.989 \text { (Mohamoud and Parmar, }\right. \\
\text { 2006), } M A Q=0.015 D A^{0.989} \text { ), and s is slope, mean of subsegments within segment. }\end{array}$ \\
\hline MIN_UNIT_SP & Calculated & Unit stream power, $\omega, \omega=\rho g Q s / w ; w=$ channel width, minimum of subsegments within segment. \\
\hline STRAHLER & Rivex 1,5 & Strahler stream order: hierarchical numbering of streams within network. \\
\hline SHREVE & Rivex $^{1,5}$ & Shreve stream order: number of upstream links. \\
\hline HRU_SEGMENT & $\mathrm{PRMS}^{1,3}$ & Same as Grid Code; links to other hydrologic modeling and temperature data \\
\hline D-LINK & $\operatorname{ArcMap}^{1,2}$ & Shreve stream order of the downstream segment. \\
\hline DLINK_STRA & ArcMap ${ }^{1,2}$ & Strahler stream order of the downstream segment. \\
\hline DS_GC & ArcMap $^{1,2}$ & Grid code of the downstream segment. \\
\hline BARRIER & $\operatorname{ArcMap}^{1,2}$ & Attributed with a 0 if it is not the site of a stream barrier. \\
\hline ISOLATED & $\operatorname{ArcMap}^{1,2}$ & Located above a barrier $1=$ yes; $0=$ no. \\
\hline BASIN & $\operatorname{ArcMap}^{1,2}$ & $\begin{array}{l}\text { Subbasin where applicable to fine models. Upper Chattahochee River Basin, Chestatee River } \\
\text { Basin, Potato Creek Basin, Ichawaynochaway River Basin, Spring Creek Basin, and Chipola } \\
\text { River Basin. }\end{array}$ \\
\hline URBAN2020 & $\operatorname{ArcMap}^{1,2}$ & $\begin{array}{l}\text { Percent of HRU with a probability of greater than } 50 \% \text { urban, HRU=watershed, corresponding to } \\
\text { each stream segment for 2010; from Urban predictions from North Carolina State University: } \\
\text { http://www.basic.ncsu.edu/dsl/urb.html (Belyea and Terando, 2012). }\end{array}$ \\
\hline URBAN2030 & $\operatorname{ArcMap}^{1,2}$ & $\begin{array}{l}\text { Percent of HRU with a probability of greater than } 50 \% \text { urban, HRU=watershed, corresponding to } \\
\text { each stream segment for 2010; from Urban predictions from North Carolina State University: } \\
\text { http://www.basic.ncsu.edu/dsl/urb.html (Belyea and Terando, 2012). }\end{array}$ \\
\hline URBAN2040 & $\operatorname{ArcMap}^{1,2}$ & $\begin{array}{l}\text { Percent of HRU with a probability of greater than } 50 \% \text { urban, HRU=watershed, corresponding to } \\
\text { each stream segment for 2010; from Urban predictions from North Carolina State University: } \\
\text { http://www.basic.ncsu.edu/dsl/urb.html (Belyea and Terando, 2012). }\end{array}$ \\
\hline URBAN2050 & $\operatorname{ArcMap}^{1,2}$ & $\begin{array}{l}\text { Percent of HRU with a probability of greater than } 50 \% \text { urban, HRU=watershed, corresponding to } \\
\text { each stream segment for 2010; from Urban predictions from North Carolina State University: } \\
\text { http://www.basic.ncsu.edu/dsl/urb.html (Belyea and Terando, 2012). }\end{array}$ \\
\hline URBAN2060 & $\operatorname{ArcMap}^{1,2}$ & $\begin{array}{l}\text { Percent of HRU with a probability of greater than } 50 \% \text { urban, } \mathrm{HRU}=\text { watershed, corresponding to } \\
\text { each stream segment for 2010; from Urban predictions from North Carolina State University: } \\
\text { http://www.basic.ncsu.edu/dsl/urb.html (Belyea and Terando, 2012). }\end{array}$ \\
\hline
\end{tabular}


Table 2-1. Segment-scale layer attributes._Continued

[FID, feature identification number; PRMS, Precipitation Runoff Modeling System; m, meters; $\mathrm{m}^{2}$, square meters; \%, percent]

\begin{tabular}{|c|c|c|}
\hline Name & Source & Definition \\
\hline URBAN2070 & ArcMap 1,2 & $\begin{array}{l}\text { Percent of HRU with a probability of greater than } 50 \% \text { urban, } \mathrm{HRU}=\text { watershed, corresponding to } \\
\text { each stream segment for 2010; from Urban predictions from North Carolina State University: } \\
\text { http://www.basic.ncsu.edu/dsl/urb.html (Belyea and Terando, 2012). }\end{array}$ \\
\hline URBAN2080 & ArcMap ${ }^{1,2}$ & $\begin{array}{l}\text { Percent of HRU with a probability of greater than } 50 \% \text { urban, HRU=watershed, corresponding to } \\
\text { each stream segment for 2010; from Urban predictions from North Carolina State University: } \\
\text { http://www.basic.ncsu.edu/dsl/urb.html (Belyea and Terando, 2012). }\end{array}$ \\
\hline URBAN2090 & ArcMap $^{1,2}$ & $\begin{array}{l}\text { Percent of HRU with a probability of greater than } 50 \% \text { urban, } H R U=\text { watershed, corresponding to } \\
\text { each stream segment for } 2010 \text {; from Urban predictions from North Carolina State University: } \\
\text { http://www.basic.ncsu.edu/dsl/urb.html (Belyea and Terando, 2012). }\end{array}$ \\
\hline URBAN2100 & ArcMap 1,2 & $\begin{array}{l}\text { Percent of HRU with a probability of greater than } 50 \% \text { urban, HRU=watershed, corresponding to } \\
\text { each stream segment for 2010; from Urban predictions from North Carolina State University: } \\
\text { http://www.basic.ncsu.edu/dsl/urb.html (Belyea and Terando, 2012). }\end{array}$ \\
\hline
\end{tabular}

${ }^{1}$ Any use of trade names is for descriptive purposes only and does not imply endorsement by the U.S. Government.

${ }^{2}$ ArcMap, version 9.3 or 10.0 (Esri, Redlands, California).

${ }^{3}$ PRMS (U.S. Geological Survey, Denver, Colorado).

${ }^{4}$ Netrace (Miller, 2008).

${ }^{5}$ Rivex (Hornby, 2010).

Table 3-1. Land-use $C$-factor assignments used in Revised Universal Soil Loss Equation model.

[RUSLE, Revised Universal Soil Loss Equation; GAP, Gap Analysis Program]

\begin{tabular}{|c|c|c|c|c|c|}
\hline \multicolumn{2}{|c|}{$\begin{array}{c}\text { Classes from } 2001 \text { National Land Cover } \\
\text { Database (NLCD) }\end{array}$} & \multicolumn{3}{|c|}{$\begin{array}{c}\text { Cover from Southeast GAP Analysis } \\
\text { Program, } 2011\end{array}$} & \multirow[b]{2}{*}{$C$ factor $^{1}$} \\
\hline Class code & Class & $\begin{array}{c}\text { Type and } \\
\text { height of } \\
\text { raised canopy }\end{array}$ & $\begin{array}{c}\text { Percent } \\
\text { canopy } \\
\text { cover }\end{array}$ & $\begin{array}{l}\text { Percent } \\
\text { ground } \\
\text { cover }\end{array}$ & \\
\hline 11 & Open water & 0.0 & 0.0 & 0.0 & 0.000 \\
\hline 24 & Developed high Intensity & 0.0 & 0.0 & 25.0 & 0.000 \\
\hline 22 & Developed low intensity & 0.0 & 0.0 & 75.0 & 0.001 \\
\hline 23 & Developed medium & 0.0 & 0.0 & 50.0 & 0.001 \\
\hline 31 & Barren land & 0.0 & 0.0 & 25.0 & 0.001 \\
\hline 21 & Developed open space & 37.55 & 25.0 & 75.0 & 0.003 \\
\hline 41 & Deciduous forest & 37.55 & 75.0 & 100.0 & 0.003 \\
\hline 42 & Evergreen forest & 37.55 & 75.0 & 100.0 & 0.003 \\
\hline 43 & Mixed forest & 37.55 & 75.0 & 100.0 & 0.003 \\
\hline 90 & Woody wetlands & 17.55 & 75.0 & 100.0 & 0.003 \\
\hline 95 & Emergent herbaceous & 2.55 & 25.0 & 100.0 & 0.003 \\
\hline 52 & Shrub/scrub & 0.0 & 0.0 & 100.0 & 0.003 \\
\hline 71 & Grassland herbaceous & 0.0 & 0.0 & 75.0 & 0.012 \\
\hline 81 & Pasture hay & 0.0 & 0.0 & 75.0 & 0.200 \\
\hline 82 & Cultivated crops & 0.0 & 0.0 & 50.0 & 0.240 \\
\hline
\end{tabular}

${ }^{1}$ The $C$ factor was estimated from the 2001 National Landcover Database (NLCD) and Southeast GAP Analysis Program's Ecological System Classification descriptions using canopy cover and height estimates and the National Resources Conservation Service table of $C$ factors for permanent pasture, rangeland, idle land, and grazed woodlands (Renard and others, 1997; Homer and others, 2004; NatureServe, 2007). 
Publishing support provided by:

Rolla Publishing Service Center

For more information concerning this publication, contact: Director, USGS Columbia Environmental Research Center 4200 New Haven Road

Columbia, M0 65201

(573) 875-5399

Or visit the Columbia Environmental Research Center Web site at: http://www.cerc.usgs.gov/ 



\section{$\frac{\mathbb{2}}{3}$}

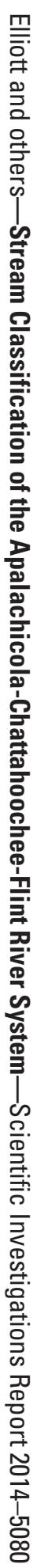

